

\section{L'auteur au temps du numérique}




\title{
L'auteur au temps du numérique
}

\author{
Audrey Llamas \\ Francesca Musiani \\ Mathieu Perona \\ Mathilde Persuy \\ Vincent Petitet \\ Marianne Serfaty \\ Guillaume Sire \\ Virginie Sonet
}

Sous la direction de:

Cécile Méadel \& Nathalie Sonnac 
Copyright @ 2012 Hadopi / Éditions des archives contemporaines

Tous droits de traduction, de reproduction et d'adaptation réservés pour tous pays. Toute reproduction ou représentation intégrale ou partielle, par quelque procédé que ce soit (électronique, mécanique, photocopie, enregistrement, quelque système de stockage et de récupération d'information) des pages publiées dans le présent ouvrage faite sans autorisation écrite de l'éditeur, est interdite.

Haute Autorité pour la diffusion des œuvres et la protection des droits sur internet (Hadopi)

4, rue du Texel

75014 Paris (France)

www.hadopi.fr

Éditions des archives contemporaines

41, rue Barrault

75013 Paris (France)

www.archivescontemporaines.com

ISBN : 9782813000880 


\section{Introduction}

La numérisation des contenus constitue une véritable lame de fond dans l'univers des industries culturelles et médiatiques, qui provoquent de multiples questionnements, en particulier des autorités de tutelle, comme l'Hadopi dans le cadre de laquelle cette étude a été conduite. De la filière du disque à celle du livre en passant par la presse écrite ou l'audiovisuelle, toutes sont touchées dans leur fonctionnement par la dématérialisation des contenus et le foisonnement de nouvelles modalités de consommation (écrans d'ordinateurs, tablettes numériques, téléphone portable, etc.). Si la dématérialisation des livres est déjà ancienne, l'impact de la numérisation est resté, au moins jusqu'à une période très récente, limité, la filière étant sans doute protégée en partie par la loi plus solidement que ne l'est la musique ou le cinéma, mais aussi plus diverse, moins concentrée, voire moins financiarisée. Trois constats illustrent ce nouvel univers :

- Le premier : les frontières se sont délitées au passage au binaire, allant jusqu'à la suppression de certaines étapes des processus de production qui font émerger de nouveaux acteurs, c'est le cas par exemple de l'agent littéraire dans la filière de l'édition.

- Le deuxième : dans cet espace reconfiguré, l'auteur a la possibilité de s'adresser directement à son lecteur, sans passer par aucun filtre, notamment celui de l'éditeur.

- Le troisième : les nouvelles technologies offrent la possibilité à tous lecteurs de devenir à leur tour créateur.

Ces trois observations ne sont qu'une illustration des potentialités de cette révolution disruptive, très en deçà sans doute de l'ampleur qu'elle pourra atteindre. 
Pour notre analyse, nous avons choisi de centrer notre réflexion sur un acteur, commun à tous les domaines de la création artistique, qui se situe en amont de chacune des filières précitées, acteur sans qui aucun processus de production ni d'industrialisation n'aurait lieu : l'auteur, entendu dans le sens très pragmatique de celui qui publie (au moins) un ouvrage papier, cet auteur fort peu étudié alors que le livre, par exemple fait l'objet de très nombreux travaux ${ }^{1}$. Nous n'entrons donc pas ici dans les débats, tant juridiques que professionnels ou philosophiques sur la définition d'un auteur, mais nous nous arrêtons à cette simple trajectoire : écrire et publier un livre.

Cet auteur se trouve face à un monde de l'écrit largement secoué :

- par la numérisation de l'information ;

- par la disponibilité démultipliée de l'information ;

- Par la "fureur d'écrire " (Méadel et Sonnac, 2012) qui touche les internautes;

- Par les bouleversements économiques du monde de l'édition et plus largement du secteur de l'information ;

Dans ce monde transformé, nous analysons ce que devient l'auteur, comment il inscrit l'acte d'écrire et de publier. La numérisation concerne plusieurs moments de la conception d'un livre et pas seulement la seule phase de l'écriture. Comme on le verra, le numérique est bien loin, pour les auteurs de livres, de se réduire à la question du piratage ; c'est même un des aspects qui les intéresse le moins, bien loin derrière les questions d'information, de diffusion, d'organisation de la chaîne du livre ou de rapport à leur éditeur.

II ne s'agit donc pas de confronter l'auteur du numérique à l'auteur de l'écrit-papier, d'opposer deux mondes, celui du numérique et celui de l'analogique, mais plutôt de s'interroger sur leur articulation. Notre étude met en lumière le fait que la frontière entre auteur-papier et auteur-

${ }^{1}$ On trouvera par exemple dans le blog tenu par Frédéric Barbier l'écho des nombreuses conférences et publications consacrées à l'histoire du livre: http://histoire-du-livre.blogspot.com/ 
numérique tend à s'éroder, tout comme s'efface la frontière entre lecture et écriture (tant augmente la propension des lecteurs à commenter). Si celle-ci semble encore visible, on s'achemine davantage vers une dialectique féconde entre édition papier et édition numérique, plutôt que vers une scission entre ces types de pratiques.

Nous explorons dans cette enquête plusieurs aspects de la "carrière" de l'auteur, entendue au sens large d'un parcours, d'une pratique, d'une façon d'être et de faire, et de manière transverse :

- d'abord, comment écrit-on en régime numérique? L'écriture elle-même avec ses outils électroniques, ses dispositifs spécifiques (couper/coller, liens, capacité de recherche dans le texte, palimpseste effacé...) ;

- comment s'opère le travail d'information ou comment l'auteur fabrique-t-il son univers de connaissances, d'informations, de références ;

- l'interaction dans le texte ou comment les auteurs font éventuellement intervenir d'autres regards, celui de l'éditeur dans son élaboration, mais aussi celui des lecteurs, des internautes ;

- ensuite, comment les nouvelles capacités de diffusion de l'information, principalement autour d'Internet, agissent sur la circulation des écrits, le rapport au public...

- puis, comment se passe la reconfiguration des intermédiaires, en particulier celle du rôle de l'éditeur dans la filière du livre, premier interlocuteur de l'auteur et un des acteurs principaux éprouvés par le numérique ;

- enfin, y-a-t-il un nouveau statut de l'auteur dans le temps du numérique.

Nous avons choisi une définition de l'auteur qui pourrait sembler restrictive par rapport à la "fureur d'écrire" évoquée plus haut, quand tant d'internautes pratiquent aujourd'hui de manière extensive cette activité si exigeante, l'écriture. Cette figure de l'auteur est ancienne puisque Michel Foucault (1994) en fait remonter la fonction au XVIe siècle lorsqu'il devient obligatoire de rajouter sur toute publication le nom de l'auteur à celui de l'éditeur qui jusqu'à 
lors suffisait à identifier une publication. Cette identification n'est plus suffisante pour définir qui est auteur et qui ne l'est pas. Nous avons donc choisi de définir l'auteur par un support qui pourrait sembler fragilisé voire compromis justement par le numérique, le livre papier. En effet, comme on le verra, le livre-papier demeure une forme privilégiée de la lecture au long cours, y compris par les pratiquants les plus intensifs de l'Internet.

II y avait autrefois (dans les temps si proches qui précèdent l'avènement du web) une coupure assez nette entre l'auteur publié et l'écrivain non publié. Un nombre semble-t-il important de gens écrivaient pour eux, pour leur famille et leurs proches éventuellement, faute de vouloir être édité ou de le pouvoir. Avec Internet, cette coupure est d'une certaine manière remise en cause dans la mesure où l'accès au public semble beaucoup plus immédiat. Pourtant, si sur le web on écrit pour les autres, dans la très grande majorité des cas (et sans doute beaucoup plus souvent encore que sur le papier), on n'est lu par personne ou presque; publier sur Internet ne donne pas de garantie de trouver le moindre lecteur (Cardon, 2006). Mais pour un auteur, le texte mis en ligne devient néanmoins (potentiellement) public, et il n'a plus le statut strictement confidentiel du journal intime ou du roman que l'on conservait à l'abri des regards.

Ont donc été interviewés pour notre enquête des auteurs qui avaient publié au moins un ouvrage-papier (de manière assez conforme à la définition qu'en donne le droit, voir l'encadré juridique ci-dessous). Une telle approche pouvait sembler être une réduction, mais elle nous permettait, de manière très pragmatique, de délimiter notre champ d'étude dans le cadre d'une enquête, par nécessité limitée, puisque celle-ci a été conduite dans le cadre des "labs Hadopi " qui ont pour vocation de produire librement et en toute indépendance débats et réflexions sur les questions liées à la diffusion numérique et à la propriété intellectuelle. ${ }^{2}$

2 Une rencontre avec les acteurs intéressés a été organisée par les labs Hadopi en juin 2011. On en trouve le compte-rendu en ligne (labs.hadopi.fr/wikis/compte-rendu-seance-de-travail-sur-le-livre-numerique). 


\section{a) Le livre papier : définition juridique}

La seule définition juridique du livre papier est une définition fiscale, qui conditionne l'application d'un taux de TVA réduit. Le livre y est défini comme " un ensemble imprimé, illustré ou non, publié sous un titre, ayant pour objet la reproduction d'une $œ u v r e$ de l'esprit d'un ou plusieurs auteurs en vue de l'enseignement, de la diffusion de la pensée et de la culture". Pour être considéré comme un livre, un ouvrage doit remplir les conditions cumulatives suivantes:

- il doit être constitué d'éléments imprimés ;

- il doit reproduire une œuvre de l'esprit - en pratique, l'ouvrage doit comporter une partie rédactionnelle suffisante permettant de conférer à l'ensemble le caractère d'une œuvre intellectuelle ou, bien qu'étant dépourvu de contenu rédactionnel stricto sensu, l'ouvrage doit être constitutif d'une œuvre de l'esprit au regard du travail éditorial important (recherche, sélection et mise en forme de données conférant à l'ensemble une homogénéité et une cohérence globale) qui a été mis en œuvre aux fins de sa rédaction (exemple : guides touristiques) ;

- en outre, il ne doit pas présenter un caractère commercial ou publicitaire marqué, c'est-à-dire être principalement destiné à informer un public de l'existence et des qualités d'un produit ou d'un service, avec ou sans indication de prix, dans le but d'en augmenter les ventes ou de promouvoir l'image d'un annonceur ;

- enfin, il ne doit pas contenir un espace important destiné à être rempli par le lecteur.

En pratique, pour l'appréciation de ces deux derniers critères, la surface consacrée à la publicité (annonces commerciales, noms de marque, logos professionnels, etc.) ou à des blancs intégrés au texte en vue d'être utilisés par le lecteur ne doit pas dépasser le tiers de la surface totale de l'ouvrage.

Cette définition quasi juridique en entraîne une autre : le périmètre de notre étude concerne tous les secteurs de l'édition. Nous considérons comme auteur toute personne ayant publié un ouvrage papier, qu'il s'agisse de poésie, de reportages, de livres d'art, de bande dessinée, de philosophie, de livre de cuisine, de nutrition ou de bricolage... Bien sûr, la littérature 
occupe une place importante dans les sorties d'ouvrages: elle représente $26 \%$ du poids dans les ventes de livres en 2010, et demeure une valeur sûre aux yeux des éditeurs et des distributeurs ${ }^{3}$. Bien sûr aussi, le genre "littérature" représente, depuis le milieu du XIXe siècle, avec tous les aléas des classifications de genre, au moins le tiers de la production (Sapiro, 2008). Mais nous avons voulu nous pencher aussi sur les deux tiers restants, et sur l'ensemble des auteurs d'ouvrages. Nous faisons l'hypothèse qu'ils proposent des façons d'être auteur, des profils et des expériences, et sans doute aussi par contrecoup une présence au numérique, qui peuvent différer de ceux de l'auteur littéraire, beaucoup plus étudié (Lahire, 2006). Nous n'examinerons pas uniquement la seule (et petite) frange professionnalisée des auteurs; si la tension entre professionnalisation et vocation, entre cité industrielle et cité inspirée pour reprendre les catégories de Luc Boltanski et Laurent Thévenot, perdure pour un certain nombre d'auteurs, en particulier les plus "littéraires" d'entre eux, la plupart de ceux que nous étudions, même romanciers, le plus grand nombre de ceux qui ont publié un livre-papier ne sont pas "en quête d'un statut ", suivant le processus pointé par Gisèle Sapiro et Boris Gobille (2006).

La population des auteurs est d'ailleurs difficile à circonscrire ; il n'y a bien sûr pas de réglementation qui régit l'entrée dans la profession. Le décompte même des auteurs est quasi impossible: il est vrai que le nombre de publications augmente chaque année approchent les soixante-dix mille ouvrages édités en 2010. Même les organismes de sécurité sociale, et plus particulièrement l'Agessa en charge des auteurs, illustrateurs de livres, écrivains, traducteurs... ne peuvent mesurer avec certitude l'effectif total de cette

\footnotetext{
${ }^{3}$ Selon l'étude réalisée par Xerfi, le livre jeunesse représente la "locomotive" du secteur avec une croissance annuelle supérieure à $15 \%$, la BD représente elle aussi un véritable moteur de croissance (sur la période 1995-2008). Les segments des ouvrages scolaires, des sciences humaines et sociales ainsi que celui des encyclopédies et des dictionnaires sont les plus touchés par la concurrence générée par les contenus multimédias. Source, Xerfi France, La distribution de livres, Analyse de marché et perspectives 2010, septembre 2010. Le Centre national du livre fait état de données semblables (http://www.centrenationaldulivre.fr/?Chiffres-cles-du-livre)
} 
population pour des raisons différentes ${ }^{4}$. Prenons l'Agessa, elle n'a d'abord connaissance que des auteurs qui perçoivent des rémunérations au titre de droits d'auteur (or un certain nombre de personnes qui publient des livres n'en perçoivent pas, qu'ils publient à compte d'auteur ou que leur contrat ne prévoit pas de rémunération ${ }^{5}$ ). Ensuite, l'Agessa n'identifie réellement que les auteurs qui sont dit " affiliés ", c'est-à-dire ceux qui touchent au moins $7893 €$ en droits d'auteur dans l'année ${ }^{6}$. Cela exclue les $94,1 \%$ d'auteurs dits assujettis ${ }^{7}$ qui touchent des droits d'auteur inférieurs à cette somme (droits non nuls), mais aussi ceux, beaucoup moins nombreux mais non dénombrables, qui gagnent plus mais qui, ayant une profession par ailleurs, négligent de s'affilier à l'Agessa8. Enfin, les déclarations des éditeurs pour les droits d'auteur de faible importance n'étant pas nécessairement nominatives, cela interdit tout recensement précis de la population des assujettis. L'Agessa peut donc fournir une estimation globale à un moment donné du nombre d'auteurs rémunérés, mais elle ne

\footnotetext{
${ }^{4}$ L'Agessa est un organisme qui recouvre pour le compte de la sécurité sociale les cotisations et les contributions dues sur les rémunérations artistiques. II est précisé que le régime de sécurité sociale des artistes auteurs est une branche du régime général des salariés, financée par les cotisations des artistes auteurs auxquelles s'ajoute une part contributive mise à la charge des personnes (physiques ou morales, y compris l'État, les établissements publics, les collectivités territoriales) qui procèdent à la diffusion ou à l'exploitation des œuvres. (http://www.agessa.org/getpage_Les-missions_9,,.html). Selon son rapport annuel (Comptes annuels 2010 de l'Agessa), 29,72\% des auteurs affiliés à l'Agessa ont un revenu annuel inférieur ou égal au seuil d'affiliation (soit 7893 euros) $45,86 \%$ ont un revenu annuel compris entre le seuil d'affiliation et le plafond de la sécurité sociale (7 893 et 34308 euros); enfin, $24,4 \%$ ont un revenu annuel supérieur plafond de la sécurité sociale (34308 euros). L'effectif total des auteurs affiliés à l'Agessa est de 12027 personnes, en 2010 (contre 11713 en 2009).

${ }^{5} \mathrm{Au}$ moins pas en dessous d'un seuil que peu atteignent.

${ }^{6}$ Auquel s'ajoutent, selon l'Agessa, environ $20 \%$ d'auteurs qui touchent moins mais sont maintenus par une commission professionnelle (en particulier pour lisser les effets temporels).

${ }^{7}$ Suivant le rapport de l'Agessa pour 2010, 12027 sont auteurs affiliés (5,9 \%) dont 2019 écrivains, 1591 illustrateurs, 934 traducteurs, 116 auteurs d'œuvres numériques, soit moins de cinq mille personnes. 191824 (94,1\%) font l'objet d'un ou de plusieurs assujettissements au cours de l'année 2010. II y aurait au total 203851 auteurs affiliés et assujettis à l'Agessa.

8 Ils en ignorent, selon un responsable de l'Agessa, très souvent l'obligation.
} 
peut comptabiliser le "stock " global des écrivains ayant au cours, par exemple des dix dernières années, reçu des droits d'auteur et encore moins de l'ensemble des "publieurs de livre-papier " Du côté des sociétés d'auteur, le pourcentage des auteurs adhérents est encore beaucoup plus faible: la Société des gens de lettres (SGDL) qui pourtant, depuis plus d'un siècle et demi, se donne pour vocation de défendre "tous les auteurs de l'écrit", en compterait six mille pour quatre mille adhérents à jour ; la Société Française des Intérêts des Auteurs de l'écrit (Sofia) qui gère le droit de prêt aux bibliothèques six mille ; la Société civile des auteurs du multimédia (SCAM) près de quatre mille, la SACD qui accueille auteurs dramatiques mais aussi de l'audiovisuel, compte plus de 50000 associés, pour 8500 sociétaires. Seule une petite partie des auteurs sont donc affiliés à ces différents organismes. C'est donc dire que la population est aussi nombreuse que méconnue, dès lors toutefois qu'on ne se limite pas à ses figures les plus reconnues ou éminentes.

En effet, une faible partie des "publieurs" inscrivent leur activité d'écriture dans une démarche professionnelle ou à vocation professionnelle. On sait par les enquêtes et la littérature économique sur le sujet que, si les auteurs ont une très large diversité d'activités et de profils, fort peu vivent des revenus directs tirés de leurs livres. C'est le cas de quatre seulement des vingt-deux que nous avons interviewés (auxquels s'ajoutent deux auteurs qui, grâce à leurs ouvrages, ont pu travailler pour des maisons d'édition). Les difficultés à vivre de sa plume sont anciennes et bien connues, les auteurs comptant rarement comme le signale Lahire $(2006)^{9}$ parmi les professionnels du livre. La rupture souvent retenue passe entre les auteurs pour lesquels l'activité d'écriture est secondaire par rapport à une activité principale qui constitue leur vocation première (dans notre échantillon il s'agira des auteurs signalés sous ces dénominations : [Policier, Roman policier, Paris], [Philosophe, Paris],

9 "Toute l'activité économique de l'édition repose, en définitive, sur le travail et les productions d'acteurs souvent "non-professionnels", qui n'y consacrent pas l'essentiel de leur temps, et qui sont obligés de trouver ailleurs les sources de leur survie économique personnelle ou familiale. " 
[Médecin, Roman policier, Paris], [Nutrition, Paris]), et ceux pour qui l'activité secondaire ne sert qu'à fournir des revenus suffisants, et qui souhaiteraient passer le plus de temps possible à l'écriture ou aux activités liées (pour nous, [Premier roman, Paris], [Romancier, Paris]). II faudrait ajouter une catégorie intermédiaire (pour nous [Philosophe, Paris]) pour laquelle l'écriture est un produit non pas indirect (comme le médecin nutritionniste) mais directement attendu de l'activité : les auteurs académiques, attendent, sauf rares exceptions, essentiellement des rétributions symboliques (en notoriété) ou indirectes (en position professionnelle, réputation et reconnaissance) plus que financières (en droits d'auteur) ${ }^{10}$.

À côté des droits d'auteurs, se sont donc ajoutés, depuis longtemps, pour les auteurs qui en vivent comme pour les autres, d'autres revenus liés à leur activité d'auteur: la commande publique, de l'État ou des collectivités locales, des bourses, des résidences d'artistes, des commandes pour la radio, des enseignements, etc. Dans notre panel, on compte parmi leurs autres activités liées à l'écriture, des travaux de communication [Romancière, Communication, Paris], de traduction et, dans le cas de la Bande Dessinée (BD) de lettrage [BD, Sud], du journalisme [Auteur-blogueur, Paris], des activités pédagogiques [Littérature enfantine, Ajaccio] ou, ce qui semble être la trajectoire la plus logique, des activités d'édition, qui placent les auteurs concernés des deux côtés de la barrière [Vulgarisation, Paris], [Homme de lettres, Paris]. Le livre publié devient, pour certains des auteurs interviewés, un élément d'un continuum d'activités, qui vont de l'adaptation (télévisée ou cinématographique) à la scène (théâtrale ou muséale), en passant par des interventions pédagogiques, journalistiques ou culturelles ${ }^{11}$. Cette pluralité n'est d'ailleurs pas nouvelle comme l'a prou-

10 Le secteur des sciences humaines et sociales (droit) compris représentait en $20097 \%$ du CA de l'édition pour $3 \%$ des exemplaires vendus ; les sciences et techniques $4 \%$ du CA pour $1 \%$ des livres vendus. (Enquête Ministère de la culture, "Économie du livre : chiffres-clefs 2009-2010 ", mars 2011).

11 On ne trouve pas d'informations sur une autre source de revenus liée aux activités institutionnelles, participations à des jurys ou festivals, à des organismes régionaux, commissions... 
vé la controverse de 1968 relatée par Gisèle Sapiro et Boris Gobille (2006) entre une conception d'écrivain comme propriétaire (de son œuvre) versus celle de l'écrivain comme travailleur (avec prise en considération de toutes ses activités qu'elles aient ou non le livre pour support).

\section{L'enquête}

Entre mars et juillet 2011, nous avons réalisé 22 entretiens approfondis, en vue de comprendre la vie des auteurs au temps du numérique. S'y ajoutent aussi l'expérience propre des rédacteurs de ce texte, les deux responsables et trois des auteurs impliqués ayant publié des ouvrages, principalement, mais non exclusivement, de type académique.

D'abord, notre choix d'auteurs s'est appuyé sur la diversité de leur profil : auteurs de romans (historique, policier, etc.), auteurs de BD, de livres pratiques, essayistes, etc., de type de production, d'âge, d'ancienneté de publication et de renom différents... Délibérément, nous souhaitions ne pas circonscrire notre analyse à la seule étude des écrivains de littérature au sens de Lahire, même si, les données sociographiques de son enquête dépassent probablement ces seuls écrivains (par leur appartenance sociodémographique, genre, faiblesse des revenus, etc.). La diversité des "segments de marché " que recouvre l'édition ne permettait pas d'avoir des échantillons de chaque catégorie dans le cadre d'entretiens non directifs; le choix des auteurs vise donc simplement un certain degré de diversité.

Ensuite, comme on l'a dit, nous avons choisi de n'interviewer que des auteurs qui avaient publié au moins un livre dans un format papier (même si cela peut être de l'édition à la carte, pour la quasi-totalité des livres-papier, mais pour certains aussi des livres numériques); sans cette condition préalable, il devenait difficile de définir un auteur, sinon à considérer que toute personne écrivant sur Internet était un auteur, ce qui risquait de nous éloigner de notre objet, le livre et étendre à l'infini notre domaine d'investigation. Notons que nous les avons contactés soit par relations personnelles, soit par Internet. 
Enfin, soulignons que l'objectif de l'étude était d'avoir des auteurs ayant publié à compte d'éditeur. Nous avons dû laisser de côté les auteurs publiant uniquement à compte d'auteur. Toutefois l'un au moins de nos interviewés a publié chez un éditeur électronique qui propose une acception un peu nouvelle, comme on le verra, du contrat d'édition, à michemin entre compte d'auteur et compte d'éditeur.

Tous les témoignages ont été anonymisés ; les auteurs sont signalés par le genre dominant de leur production d'auteur (même si la plupart des auteurs publient des ouvrages de différents genres), de leur genre (homme ou femme) et de leur lieu de résidence ${ }^{12}$.

Ce corpus est donc relativement inédit, non par les questions qui lui sont adressées mais par la fonction qu'il occupe dans l'étude d'un champ aux pratiques encore peu étudiées. C'est la raison pour laquelle nous avons choisi de laisser très largement la parole aux auteurs, ce maillon trop négligé de la chaîne du livre. Ce passage au temps du numérique redonne à l'auteur une place centrale dans la filière de l'édition en général, et au sein de notre ouvrage en particulier.

Notre analyse comporte trois chapitres. Le premier chapitre s'intéresse au travail de l'auteur. Comment travaille-t-il et dans quelle mesure la numérisation des contenus influencet-elle l'écriture, la conception des textes, la pratique de l'écrivain, voire la fabrication du livre lui-même. Le deuxième porte sur le stade ultérieur, le moment où le manuscrit se détache de l'auteur pour entrer dans la sphère publique, se faire connaître et reconnaître. Cette phase de diffusion est celle qui est la plus directement influencée par l'émergence du web, la multiplication des moyens de communication, l'efflorescence de lieux d'échanges. Le troisième enfin met la focale sur l'alter égo de l'auteur, celui auquel il confie à la fois son livre et sa diffusion, l'éditeur. On s'intéressera en particulier aux attentes de l'auteur à son égard, à la manière dont il perçoit les obligations et droits de son contrat pour

12 On en trouvera la liste en annexe 3. 
terminer par les modifications induites ou attendues de l'âge numérique. On trouvera, au fil du texte, quelques encadrés qui apportent un autre éclairage sur les thèmes abordés, en particulier un certain nombre de définitions juridiques qui précisent les notions liées aux droits des auteurs et à la propriété intellectuelle ${ }^{13}$.

13 Définitions juridiques pour lesquelles nous remercions Christophe Alleaume et Mathilde Persuy, du labs Propriété intellectuelle et internet de l'Hadopi. 


\section{Chapitre 1:}

\section{Écrire en régime numérique}

Dans son article Is Google Making Us Stupid?, Nicholas Carr s'est interrogé sur les effets des technologies de l'information sur la manière dont nous lisons et dont nous percevons les informations. II cite à des fins d'illustration l'exemple du philosophe Friedrich Nietzsche qui, Iorsqu'il acquit sa première machine à écrire, changea son rythme d'écriture, son tempo, son phrasé, sa musicalité, si bien que cette nouvelle manière d'écrire finit par "emporter " le philosophe là où il n'était pas encore allé : Nietzsche changea, dit-il, sa manière de raisonner "Selon l'écrivain et compositeur Heinrich Köselitz, sa prose était devenue plus ramassée, plus télégraphique, (..), comme si la puissance de sa machine s'était par un certain mécanisme métaphysique mystérieux, transposée dans les mots qu'elle imprimait sur la page "14. Cet exemple pose des questions intéressantes: comment la technique interfère-t-elle dans les pratiques d'écriture au point d'agir sur les pratiques de pensée? Jack Goody [1979] a consacré son important travail sur l'écriture à cette question analysant la manière dont les modus operandi, les instruments de l'écriture façonnent l'ingénierie intellectuelle ou, pour le dire autrement, comment les outils utilisés par l'auteur influencent et contribuent à définir la connaissance et la pensée. L'écriture est concomitante avec l'évolution des sociétés et les représentations discursives des individus. Dès lors, toute évolution d'une forme

14 Carr, Nicholas, Internet rend-il stupide ?, Paris, Robert Laffont, 2011, p.41. 
d'écriture a des conséquences majeures, au niveau macrosocial, dans l'épistémè d'une société, mais aussi au niveau microsocial au cœur des processus cognitifs. De fait, dans quelle mesure une innovation peut-elle changer le rapport d'un auteur avec son travail (la pratique) et avec le résultat de ce travail (l'objet-livre) ? L'informatisation, puis l'usage du réseau Internet, en somme la numérisation, ont-ils eu un impact sur la manière d'écrire et de concevoir l'acte d'écrire? Sur l'objet-manuscrit? Sur l'objet-livre ? Sur la perception qu'ont les auteurs de ces objets ? Autrement dit, dans quelle mesure la numérisation a-t-elle constitué une rupture dans le rapport entre l'écrivain, sa pratique et son objet, jusqu'à quel point cette reconfiguration sociotechnique bouleverse-t-elle l'organisation existante?

Á partir des entretiens réalisés, nous nous proposons d'interroger la façon dont les auteurs mobilisent le numérique dans leurs pratiques de travail, et les éventuelles répercussions qu'ont ces nouvelles pratiques sur la relation qu'ils ont avec l'objet : sa fabrication, son statut, les caractéristiques semblant être "invariables " et celles qui sont sujettes à des mutations plus ou moins profondes. Nous serons ainsi à même de nous demander si l'informatique a changé la manière d'écrire de certains auteurs, et, si tel est le cas, dans quelle mesure. Nous verrons également quel usage les auteurs font d'Internet pour s'informer, se documenter et enrichir leurs propos. Nous chercherons ainsi à savoir si les auteurs qui s'approprient l'outil informatique et font un large usage du réseau parlent de continuité ou de rupture. Nous verrons que cette filiation ou cette rupture ne peuvent pas être considérées comme radicales compte tenu de la nature ambiguë très largement partagée des sentiments entretenus par les auteurs à l'égard du numérique. Tous ceux que nous avons rencontrés se servent de l'outil informatique mais à des stades plus ou moins avancés et à des échelles plus ou moins grandes. Tous ont publié au moins une version papier de leurs ouvrages (condition initiale que nous avons fixée pour définir l'auteur, comme nous l'avons dit en introduction), mais pour des raisons et des besoins qu'ils justifient différemment. Tous utilisent le ré- 
seau Internet, mais de manière plus ou moins régulière, " éclairée " ou " simple ", profane ou geek.

Dans une première partie, nous verrons comment les auteurs "fabriquent" leurs livres: comment ils écrivent d'abord et travaillent le texte, comment ils finalisent le manuscrit, produisant une première version de l'objet qui devra être communiquée aux responsables de l'édition, puis comment ils interviennent dans la fabrication du livre en tant qu'objet fini, sa maquette, sa conception, sa couverture. Cela nous permettra de nous interroger en conclusion sur un éventuel dialogue des supports papier et numérique, d'abord dans la pratique d'écriture, tâche qui s'effectue essentiellement seul, puis en matière d'implication dans la fabrication de l'objet, généralement un travail de groupe.

Dans une seconde partie, nous étudierons la conception que se font ces auteurs du livre à l'heure du numérique. Nous verrons ce qu'ils pensent du "livre enrichi " et de l'e-book et si, pour eux, il y a reconfiguration, continuité ou continuité assortie d'une amélioration.

Enfin, nous essaierons de voir comment les auteurs " habitent " l'espace-temps Internet: les auteurs ont évoqué deux principales formes d'utilisation: les échanges qu'Internet facilite ou même favorise et les savoirs dont la disponibilité s'est considérablement accrue. On verra donc ce qu'Internet permet aux auteurs en termes d'échanges avec d'autres auteurs, d'autres textes, éventuellement avec les lecteurs, et comment ils utilisent la Toile pour se documenter.

\section{1 Écrire et fabriquer un livre}

\section{La démarche d'écriture}

La démarche d'écriture qui utilise aujourd'hui presque toujours de nouveaux outils numériques est une activité exigeante qui mobilise son auteur. La présence de l'ordinateur semble désormais quasi inhérente à la démarche des écrivains, même si certains revendiquent davantage une dynamique d'aller-retour entre support nu- 
mérique et support papier. Dans tous les cas le processus d'écriture n'a rien de figé et se réalise dans une complémentarité entre les supports.

Une activité exigeante...

Nos entretiens ont montré que l'acte d'écriture était souvent perçu par les auteurs comme un périple solitaire, fastidieux et exigeant, dont la réussite dépend d'un équilibre fragile et d'une maturation chronophage. Comme le dit un jeune auteur : " C'est solitaire, c'est long ".

Cette solitude concerne principalement les auteurs littéraires; pour d'autres le travail se fait en collaboration avec d'autres auteurs, ce presque systématiquement dans le cas des livres illustrés, des dictionnaires, des bandes dessinées, éventuellement des essais, des livres pratiques... Cette collaboration peut être totale comme cette femme qui écrit avec son mari des livres de vulgarisation et conçoit le travail comme entièrement commun. Elle peut être inégalement répartie, comme les illustrateurs qui n'interviennent parfois qu'en fin de parcours, lorsque le livre est entièrement rédigé. Elle peut également être spécialisée mais structurante comme ces ouvrages où le dessin est conçu en parallèle du texte (dans les bandes dessinées ou les livres pour enfants par exemple).

\section{b) L'œuvre en collaboration ou œuvre plurale}

L'écriture en mode collaboratif peut donner lieu à plusieurs types d'œuvre plurale ${ }^{15}$ : "Est dite de collaboration l'œuvre à la création de laquelle ont concouru plusieurs personnes physiques. Est dite composite l'œuvre nouvelle à laquelle est incorporée une œuvre préexistante sans la collaboration de l'auteur de cette dernière. Est dite collective l'œuvre créée sur l'initiative d'une personne physique ou morale qui l'édite, la publie et la divulgue sous sa direction et son nom et dans laquelle la contribution personnelle des divers auteurs participant à son élaboration se fond dans l'ensemble en vue

15 régie par les articles L. 113-2 et suivants du code de la propriété intellectuelle 
duquel elle est conçue, sans qu'il soit possible d'attribuer à chacun d'eux un droit distinct sur l'ensemble réalisé ".

Sont donc distinguées les œuvres créées simultanément par plusieurs auteurs (œuvres de collaboration et œuvre collective) et les œuvres qui intègrent une œuvre existante réalisée par un tiers sans que celui-ci soit associé à la création de l'œuvre nouvelle - on parle alors d'œuvres composites. Ces différentes œuvres peuvent intégrer des contributions d'un genre différent, tel le graphisme et les textes pour une bande dessinée, par exemple.

L'œuvre de collaboration, comme la bande dessinée, réalisée par des personnes physiques animées par une inspiration commune, est la propriété indivise de ses auteurs. Chaque auteur est en outre libre d'exploiter séparément sa propre contribution, dans la mesure où elle relève d'un genre différent (dessin/texte) et que cette exploitation n'a pas été interdite par une convention contraire ni ne porte atteinte à l'exploitation de l'œuvre commune.

L'œuvre collective, comme la réalisation d'un journal ou d'un dictionnaire est, quant à elle, la propriété de la personne (le plus souvent morale) qui a initié et encadré sa création et qui l'exploite en son nom : le régime de l'œuvre collective permet la rémunération de celui qui a supporté les risques de la création.

L'œuvre composite est une œuvre nouvelle et originale qui incorpore une œuvre existante. C'est le cas par exemple, de l'incorporation d'une musique dans un film publicitaire ou encore de l'incorporation de photographies dans des documents publicitaires. L'absence de participation de l'auteur de l'œuvre existante à l'œuvre nouvelle exclut la qualification d'œuvre de collaboration. L'accord de l'auteur de l'œuvre incorporée devra être requis si cette œuvre fait encore l'objet d'une protection.

Les auteurs organisent leur emploi du temps en fonction de ces contraintes d'écriture; on parle, dans des cas limites, "d'obsession", mais il est intéressant de noter que cela n'est pas réservé aux auteurs littéraires; dans le livre pour enfant comme dans la littérature pratique, la BD ou d'autres genres, l'écriture s'avère une activité à haute intensité, une tâche extrêmement prenante, l'adjectif n'est pas employé de manière indifférente, tant est présente chez les auteurs 
cette idée d'une prise, d'une dépendance, dès lors que l'on veut mener à bien le projet d'écriture.

II commence à 7 heures du soir et finit à minuit, parfois il reprend à 3 heures du matin! Cela ne cesse jamais. D'autant que là on a un très gros contrat d'édition avec mon mari (corédacteur de plusieurs livres de vulgarisation à succès) que nous sommes en train d'achever, et qui nous achève aussi ! Pendant trois ans on n'a fait que ça. Le 31 décembre j'étais en pyjama en train d'écrire. C'est le travail d'un auteur c'est-à-dire qu'il ne faut pas faire autre chose quand vous faites un livre. II faut être vraiment dans votre livre, il faut être accaparé par le sujet, l'engloutir parce qu'il y a continuellement des idées qui vont et viennent, des thèmes ou des idées que l'on veut reprendre après 6 mois... Donc on a continuellement un regard sur soi. [Vulgarisation, Paris]

L'écriture se conçoit donc comme une discipline au sens où elle impose un agenda avec une distribution particulière du temps et une mobilisation permanente des ressources de l'individu. L'auteur est dans une posture de qui-vive: il y a comme une correspondance entre son environnement et son activité d'écrivain qui le sollicite en permanence et nourrit à un rythme plus ou moins spontané son propre travail. D'où l'importance des supports de maturation: carnets de notes, feuilles éparses, enregistrement d'idées sur un téléphone portable, notes sur une tablette, etc. Tout un dispositif croisant support papier et support numérique se met en place, participe à la production du livre et à sa lente maturation dans un rapport à la création protéiforme. La question qui se pose pour l'auteur est bien de fixer une idée, une intuition et cela ne peut se faire que via un support matériel, numérique ou non :

Je vois les technologies informatiques comme un prolongement d'un rapport à la création qui est multimédia mais qui l'était déjà avant le numérique [Homme de lettres, Paris]

Le niveau d'exigence des auteurs vis-à-vis de ce qu'ils écrivent, avant même qu'il soit question de le publier, est souvent élevé. Ceci explique que, même les auteurs pour qui l'écriture n'est pas l'activité principale, c'est-à-dire la majorité d'entre eux, y consacrent beaucoup de temps, régulièrement, quitte à ce que cela empiète sur leurs autres activités, 
quitte même à organiser leur activité professionnelle principale en fonction de leur rythme de production.

La première étape c'est un travail régulier, sur plusieurs mois, trois jours par semaine. Cela est possible parce que je ne travaille jamais le vendredi, cela est entendu avec mon entreprise, je reste disponible éventuellement par téléphone parce qu'avec mon travail on ne peut pas couper tout à fait, mais je n'y suis pas. Et j'écris. J'ai toujours fonctionné comme ça. Et puis, très curieusement, lorsque j'arrive à peu près à la moitié, ou aux trois quarts, du livre, tout à coup il y a une espèce d'accélération et je pars m'enfermer une semaine, deux semaines, trois semaines, et là, je ne fais que ça, et quand je reviens à Paris, c'est pour faire autre chose, pour me reconnecter à une vie "normale "... Donc pour résumer, il y a une régularité du travail jusqu'à un certain point, puis une espèce de précipitation comme à la fin d'un opéra de Rossini. À un certain moment, on ne peut plus rien faire d'autre que ça, il y a une espèce d'obsession, il faut y arriver avant de... mourir. [Littérateur, Paris]

J'écris un livre en deux ans environ, donc j'ai commencé à 26 ans, vous calculez, j'en ai fait sept en tout, plus un recueil de nouvelles et une pièce de théâtre. C'est un rythme... Certains font du football le samedi matin, moi j'écris, c'est pris sur le loisir. [Romancier, Picardie]

Je le fais le soir et le week-end, parfois j'avoue aussi pendant mon travail. [...] Ca dépend beaucoup des périodes mais pendant les périodes où j'écris beaucoup ça peut être $20 \mathrm{~h} / \mathrm{semaine}$ quand je travaille en même temps, en vacances ça peut être plus. Mais je peux aussi ne pas écrire pendant 3 semaines 1 mois. [Premier roman, Paris]

\section{...sans rupture avec le numérique...}

La plupart des auteurs interrogés ne semblent pas se sentir différents de leurs prédécesseurs de l'avant-numérique. Si leur pratique diffère pour certains sur le plan technique, concernant l'initiative originelle, l'idée d'écrire, aucune rupture forte n'apparaît dans nos entretiens.

Je dirais que je ne vois pas de différence fondamentale entre le travail de cette époque-là [l'avant numérique] et ce qui se fait maintenant. C'est une autre façon de faire voyager le travail, de le faire connaître ; autrefois par exemple, nous recevions beaucoup de courrier, aujourd'hui ce sont les mails ; c'est donc une 
évolution, mais pas une révolution; toutes ces choses existaient déjà avant le numérique. [Homme de lettres, Paris]

On retrouve cette idée d'une certaine forme d'ascèse chez des auteurs de tous temps ${ }^{16}$. Sur leur pratique d'écriture, ils tiennent des propos qui auraient tout à fait pu être tenus avant le passage au numérique, notamment au sujet de ce qui les conduit d'une simple idée (je pense à écrire) à une véritable entreprise (j'écris).

Je travaille beaucoup mentalement avant de passer à l'écriture. C'est-à-dire, je peux très bien écrire à un feu rouge. Je n'écris pas, j'écris dans ma tête. J'écris trois phrases pour juger d'un style, et puis je les oublie et puis elles reviennent, donc il y a une préparation mentale comme ça. Chacun a ses méthodes mais globalement, je ne démarre jamais un projet littéraire sans avoir le début et la fin dans ses grandes lignes, un tracé et les personnages. [Romancier, Picardie]

II y avait certaines choses qui me touchaient, sur lesquelles j'avais envie d'écrire et donc si j'écrivais parfois des bouts et des bouts de phrases, des paragraphes, mais plus pour moi sans que cela ait vraiment de sens. À partir d'un moment j'ai pensé que certaines choses pouvaient être intéressantes. J'aime lire, j'aime écrire. Á partir d'un moment j'ai senti que j'avais de quoi écrire un roman donc je me suis lancé. [Premier roman, Paris]

Une fois la démarche entreprise, il semble que dans la majorité des cas, l'écriture elle-même (et non l'idée d'écriture par essence immatérielle) soit à destination du papier. Finalement, peu nombreux sont les auteurs qui nous ont dit être pour le "tout numérique". Ils voient le papier comme l'aboutissement, et dans certains cas une manière de couronnement, de l'entreprise (j'ai décidé d'écrire) et de l'acte effectué (ensuite, j'ai écrit). Seule, une minorité s'est lancée depuis le début dans un projet véritablement numérique, voyant alors le papier comme une finalité éventuelle, mais pas obligatoire.

16 " Qu'une chose soit difficile doit nous [les poètes] être une raison de plus pour l'entreprendre " (Lettre à une jeune poète, Rainer Maria Rilke). "L'écriture est une occupation solitaire qui accapare votre vie." (Les Revenants, Paul Auster) 
En version PDF, ce livre n'a pas de taille limite prévue. Au début du projet, je me disais: "quand il atteindra entre 150 et 200 pages, nous penserons à en faire une version papier“. [Directeur d'ouvrage collectif]

Le numérique a également pu être considéré par certains comme une étape intermédiaire ou un produit d'appel pour le papier :

La première édition d'A... était une version numérique gratuite, publiée, avec l'accord de mon éditeur, Ace and Orbit, sous licence Creative commons, avec des restrictions, comme par exemple l'interdiction de modifier, transformer ou adapter l'œuvre ou de l'exploiter dans une perspective commerciale. II y a un message sur mon site Internet disant qu'aussi bien mon éditeur que moi-même espérons que si les lecteurs ont apprécié la version numérique du livre, ils envisageront d'acheter un exemplaire de la version papier, mais c'est le seul rappel qu'on fait. Je ne suis pas tellement porté sur les rappels des partagiciels (nag screens ${ }^{17}$ ). Sur mon site, A... est disponible en HTML, avec le moins de balises possibles pour que les moteurs de veille le lisent et l'intègrent facilement. Des versions numériques gratuites sont également disponibles sur plusieurs plateformes et sous différents formats comme le RTF, l'ePub et MobiPocket. Et pourtant, c'est seulement ensuite qu'A... a été publié aux États-Unis par Ace, en livre relié et broché18; et par Orbit au Royaume-Uni, également dans les deux versions. [Science-fiction, Angleterre] ${ }^{19}$

\section{c) Creative Commons}

Le projet Creative Commons, organisation américaine à but non lucratif, est né à l'université de Stanford aux États-Unis, notamment grâce aux travaux du Professeur Lawrence Lessig (1999). De la même façon que les logiciels libres, ces licences visent à "enrichir le patrimoine commun " en offrant de nouvelles conditions de gestion du droit d'auteur destinées à

\footnotetext{
17 II s'agit de logiciels qui rappellent régulièrement à l'utilisateur que la copie dont il dispose n'est pas enregistrée et qui l'appellent à s'inscrire et payer le montant de la licence.

18 Dans l'édition en langue anglaise, la plupart des ouvrages peuvent être publiés simultanément par le même éditeur en hardcover ou hardback (version reliée) et paperback (version brochée), le prix du premier étant beaucoup plus élevé que celui du second.

19 Traduction des entretiens en anglais par Marianne Serfaty
} 
favoriser la libre diffusion des œuvres sur Internet sans déposséder les auteurs de leurs droits (Dulong de Rosnay, 2007). Le dispositif Creative Commons propose six licences types, traduites et adaptées "de la lettre et de l'esprit du contrat " américain, qui combinent un contrat, son résumé via des icônes et des technologies de marquage numérique (ou métadonnées). Chaque licence propose différentes options (absence d'autorisation d'une utilisation de l'œuvre à des fins commerciales, possibilité ou non de diffuser une adaptation de l'œuvre, etc.). Le système explique que "Les licences Creative Commons viennent en complément du droit applicable, elles ne se substituent pas au droit d'auteur. "Si plusieurs décisions de justice aux Pays-Bas (mars 2006), en Espagne (février 2006) et en Belgique (2010) les assimilent à des véritables contrats d'auteur, à l'heure actuelle, ce n'est pas le cas de la jurisprudence française. Á noter cependant que l'article L. 122-7-1 du code de la propriété intellectuelle, introduite par la loi dite "DADVSI " du 1er août 2006, pose que "l'auteur est libre de mettre ses œuvres gratuitement à la disposition du public sous réserve des droits des éventuels coauteurs et ceux des tiers ainsi que dans le respect des conventions qu'il a conclues". Selon les travaux parlementaires, cette nouvelle disposition avait pour vocation principale de reconnaître la licéité du "libre".

Trois remarques :

Dès lors que la mise en disposition d'une œuvre sous Creative Commons est par principe non exclusive, en droit, l'auteur peut parallèlement céder les droits d'exploitation (numérique et/ou papier) à un éditeur, sous réserve bien sûr que celui-ci accepte ce double statut.

Comme l'explique Creative Commons France, l'auteur qui souhaiterait, après avoir mis ses œuvres à disposition, faire cesser leur diffusion sous licence Creative Commons, pourra difficilement les faire disparaître du réseau et la "révocation " d'une licence CC sera sans effet sur "les exemplaires des œuvres qui circulent ou auront déjà été distribuées, y compris dans des adaptations ". La mise en œuvre du droit de retrait (qui permet à l'auteur ayant cédé ses droits patrimoniaux de faire cesser contre indemnisation préalable - l'exploitation de l'œuvre dont il 
souhaiterait aujourd'hui qu'elle ne fût pas divulguée ${ }^{20}$ ) reste largement théorique

Les licences sont incompatibles avec les actuels statuts des sociétés de perception et de répartition des droits d'auteur françaises et européennes (CFC et SOFIA principalement), qui imposent à l'ayant-droit sociétaire d'apporter à la société la gestion de l'ensemble de ses œuvres. L'utilisation de la licence Creative Commons est alors impossible pour les titulaires de droit qui se sont préalablement engagés envers ces sociétés.

Seul un auteur ne considérait pas du tout la possibilité de publier ses écrits sur papier. II explique s'être senti comme un "imposteur " lorsque, finalement, il a accepté qu'un éditeur publie ses billets. Dans notre enquête, ce cheminement du blog vers le papier demeure assez marginal. Nous verrons par la suite ce qui semble différencier cet auteur des autres interviewés.

J'ai commencé mon blog il y a 7 ans, j'avais 31 ans à l'époque. L'idée était d'exprimer mon expérience, mon vécu du métier, j'étais fatigué. Le blog était pour moi une bonne manière de raconter ce que je vivais, tout en "fictionnalisant " un peu pour ne pas que les gens puissent se reconnaître, mais globalement tout était vrai et j'avais besoin de le raconter, il n'y avait pas de groupe de paroles. Ce blog a rapidement très bien marché et j'ai été démarché par plusieurs éditeurs au bout d'un mois et demi, deux mois. Au début, j'ai refusé, je ne voyais ce que j'allais faire là-dedans, et puis au troisième ou quatrième j'ai fini par accepter, j'étais amusé, je me sentais comme un imposteur. Je l'ai fait aussi car beaucoup de gens le demandaient sur le blog. [Auteur-blogueur, Paris]

Pris à son tour par l'écriture, mais toujours attentif au support et aux expérimentations numériques, ce même auteur a

${ }^{20}$ Article L. 121-4 du code de la propriété intellectuelle: " Nonobstant la cession de son droit d'exploitation, l'auteur, même postérieurement à la publication de son œuvre, jouit d'un droit de repentir ou de retrait vis-à-vis du cessionnaire. II ne peut toutefois exercer ce droit qu'à charge d'indemniser préalablement le cessionnaire du préjudice que ce repentir ou ce retrait peut lui causer. Lorsque, postérieurement à l'exercice de son droit de repentir ou de retrait, l'auteur décide de faire publier son œuvre, il est tenu d'offrir par priorité ses droits d'exploitation au cessionnaire qu'il avait originairement choisi et aux conditions originairement déterminées. " 
ensuite publié un roman-feuilleton sur iPhone, entièrement en numérique, mais, plutôt que de s'en contenter, il a fini par porter ce roman-là également chez un éditeur, comme si le papier demeurait pour lui une finalité.

J'ai fait un roman pour iphone. J'ai été contacté par un éditeur d'application qui voulait tester la distribution d'un roman, vendu chaque semaine. Comme j'étais libre en ce qui concerne mes droits numériques, je me suis lancé. On a donc sorti 3 chapitres par semaine pendant 12 semaines. Ça a cartonné. Une fois cette étape terminée, je suis allé proposer le livre et un projet numérique chez Plon, qui a refusé en me disant "on n'édite pas des serpillières", sous-entendu des choses sur lesquelles des gens sont déjà passés. En fait, je me suis rendu compte qu'ils ne comprenaient pas comment saisir ce truc. [Auteurblogueur, Paris]

Une autre auteure a commencé par publier un livre en ligne avant de le reprendre, de le réécrire, pour chercher à le publier ensuite, selon son expression, "en vrai". Ainsi, il semble que le papier soit considéré par les auteurs comme une finalité. La plupart écrivent avec, en tête, l'idée d'un objet-fini : le livre physique. Ceux qui ne le font pas utilisent le numérique sans perdre de vue qu'éventuellement, une version papier pourra ensuite être publiée, la considérant alors comme un produit "plus fini " que ne l'est la version numérique. Un seul auteur qui a écrit sur un blog sans jamais penser publier ses textes en version papier a d'abord éprouvé une gêne à cette idée, sans pour autant se sentir embarrassé par l'idée de mettre ses textes en ligne, comme s'il considérait le papier comme un matériau plus noble, auquel seuls certains textes et certains auteurs sont destinés a contrario du web où l'auteur peut éprouver le sentiment d'une plus grande liberté, ne dépendant que de soi voire des lecteurs, avec une durée de vie des textes inconnue, une stabilité dans le temps qui semble moindre que celle du papier, un lectorat à la fois proche et volatile. Une fois reconnu en tant qu'auteur, il a poursuivi la publication de ses textes dans une version papier, y compris un texte publié initialement en version numérique. 


\section{... mais avec des outils différents}

Même s'il n'est pas toujours question de numérique au moment de prendre la décision d'écrire, il en est question immédiatement après, lorsque, véritablement, l'auteur entreprend d'écrire. Nous avons en effet constaté la présence d'un ordinateur dans la démarche d'écriture de chaque écrivain interrogé ; aucun ne nous a dit écrire uniquement à la main ; aucun, a fortiori, dans notre échantillon n'écrit à la machine. Tous utilisent le réseau Internet (entre autres) pour s'informer et l'outil informatique pour écrire: ils fabriquent le tapuscrit qu'ils rendront à l'éditeur, puis, éventuellement, participent à la fabrication du livre luimême.

J'écris sur l'ordinateur, puis je corrige sur ordinateur et, après plusieurs relectures/corrections sur ordinateur, j'imprime et je corrige à la main. II y a un vrai dialogue des supports. Je n'aurais pas la même chose au final si j'écrivais et corrigeais seulement sur ordinateur ou si j'écrivais et corrigeais seulement à la main. [Romancier, Paris]

La majorité des auteurs interviewés établissent ainsi un dialogue, partageant le plus souvent leur travail entre l'écriture manuelle et l'écriture sur l'outil informatique, puis les corrections manuelles et les corrections sur l'outil informatique. Des allers retours se font, que certains d'entre eux jugent indispensables à l'équilibre global de leur travail, comme si chaque support avait quelque-chose à " apporter" au texte fini que n'était pas capable d'apporter l'autre support, autrement dit, comme si l'écriture et les corrections apportées - i.e. le travail de l'auteur - étaient affectées par la nature du support. Mais cela ne signifie pas que les supports sont inconciliables, au contraire: chacun apporte un " plus " pour que le rendu soit plus abouti qu'il ne l'aurait été si l'écrivain n'avait utilisé qu'un seul des deux supports. Pour eux, cela signifie que le travail numérique n'est pas une rupture mais bien un progrès qui vient améliorer le travail manuel - progrès grâce auquel les auteurs atteignent une qualité d'écriture qu'ils jugent plus satisfaisante que s'ils avaient travaillé uniquement sur le numérique ou uniquement à la main. Pour reprendre, si l'on nous passe l'uchronie, l'exemple de Nietzsche, il aurait été intéressant 
de comparer le style et la pensée du philosophe écrivant à la main, du philosophe écrivant à la machine, et du philosophe écrivant à la main ET à la machine, et de lui demander lequel des textes il jugeait le plus satisfaisant. S'il avait annoncé qu'il préférait le texte écrit de deux façons, le jugeant plus "proche " de sa pensée, il aurait alors confirmé I'hypothèse suggérée par nos interviews.

J'écris sur un ordi. D'abord je structure mes idées dans un carnet ; j'ai besoin d'écrire les mots qui s'enchaînent ; un mot en appelle un autre. Ces mots dans des carnets me fait démarrer l'imaginaire. Parfois un mot déclenche autre chose. J'écris dans ces carnets 10X15. Après je prends un carnet plus grand, format demi A4, dans lequel j'écris au stylo plume et ce n'est plus du tout la même relation. Je vais moins vite, mais je m'applique à étirer cette idée. Et après, je sens qu'il faut que j'écrive ; j'ai mes carnets à côté de l'ordinateur que je n'ouvre pas. Je tape à l'ordinateur et en même temps, j'ai une feuille et un stylo et je note à toute vitesse des trucs [Policier, Roman policier, Paris].

Toute la première partie se passe par email. Pour moi en tout cas. Le premier jet est électronique, mais j'ai besoin d'un tirage papier pour une deuxième lecture. Les corrections ne sont pas les mêmes. Le papier est plus proche du livre, on le corrige comme un livre. Avec l'électronique, on est plus éloigné, à cause du copier-coller qui influe beaucoup. Mes manuscrits sont très travaillés mais quand même. Je fais un premier jet, c'est l'échafaudage du roman : je jette quatre ou cinq idées de dialogues, les personnages, j'essaye d'avoir la matrice du roman. Puis je travaille sur le fond. [Médecin, Roman policier, Paris]

Plusieurs de nos interviewés continuent d'écrire d'abord à la main. Outre, le besoin d'utiliser des supports différents qui jouent des rôles distincts dans le processus d'écriture, ces auteurs évoquent le plaisir qu'ils éprouvent à écrire sur du papier qui leur offre un rapport plus direct, plus tactile avec le support, sans la médiation d'un écran.

Jusqu'ici j'écris d'abord la première version papier, et après je passe à l'ordinateur, la version peut être très différente d'ailleurs (...) jusqu'ici il y avait un premier jet à la main, une reprise à l'ordinateur et un travail d'affinage que je peux faire le soir, j'imprime et relis. C'est plusieurs fois, il est important de 
prendre le temps, laisser reposer le texte pendant un ou deux mois, de ne pas écrire du tout et de le reprendre. Là je commence à travailler un peu plus en direct, sur l'ordinateur, j'ai essayé pour voir, mais il y a certains passages que j'écris à la main, parce que ça va plus vite, et puis selon la tonalité du passage, on a besoin de se mettre dans certaines conditions, on veut aller plus vite, ne pas être freiné par la technologie. C'est aussi un plaisir d'écrire à la main, un vrai plaisir physique, sensuel de voir la feuille... que l'on n'a pas avec l'ordinateur. [Romancier, Picardie]

Le texte est écrit chapitre par chapitre, à la main. Dès que j'ai terminé un chapitre, je recopie à la machine (l'ordinateur) chapitre par chapitre. Et je n'écris le chapitre suivant à la main que lorsque le chapitre entré dans l'ordinateur est pour moi dans une forme acceptable. II y a donc déjà une première étape de correction qui s'opère entre le manuscrit et le passage à l'ordinateur. C'est comme quand on quitte sa chambre, et qu'il faut avoir fait son lit, je ne pars pas dans un nouveau chapitre tant que tout, derrière moi, n'est pas bien en ordre. [Littérateur, Paris]

Pour cet auteur, universitaire, il y a moins dialogue des supports entre le numérique et le manuel que "va-et-vient fastidieux " entre les deux; le numérique est requis d'abord pour ses capacités de mémoire : c'est lui qui permet de garder la trace des versions successives du texte manuscrit :

Je suis à la fois papier et numérique, je fais les deux! Quand j'écris désormais, cela commence par des mots éparpillés sur une feuille de papier; rarement deux. Ou alors des bouts de papier, en épluchant des patates ; j'écris comme une envie de pisser. Je note ça sur des enveloppes et quand je me mets à écrire, à partir de ces petits morceaux de papier, j'écris une version numérique que j'imprime. Puis, il y a un va-et-vient long et fastidieux entre le numérique et le manuel. J'imprime, je corrige à la main au stylo rouge ; puis je le retape ; je le sauvegarde avec la date (comme un palimpseste ou plutôt un millefeuille numérique des différentes étapes). Quelques-uns de mes textes numériques sont écrits d'une traite ; avec une seule série de corrections. Les textes vraiment travaillés subissent des allers retours importants entre numériques et manuels. Au moins une vingtaine de version. 
- Pourrait-on se passer du papier?

- Moi, non. J'en ai besoin. Je corrige en rouge. II y a peut-être des auteurs qui s'en passent. [Philosophe, Paris]

Pour un autre auteur, le livre-papier reste la référence idéale au point qu'il a besoin d'en avoir une représentation homothétique pour mieux voir ce qui ne va pas dans ce qu'il a écrit, pour faire les corrections :

Quand j'imprime des chapitres, c'est déjà en format livre, parce que je trouve que quand on imprime avec un texte en page au format d'un livre, les fautes sautent plus au visage que lorsque c'est écrit au kilomètre sur une feuille A4. En plus, avant de passer à un nouveau chapitre, non seulement je relis le précédent, mais en plus, je relis tous les autres, pour être bien en phase... Pour résumer, je l'écris à la main, je le corrige, je le tape à l'ordinateur, je le corrige, je l'imprime, je le corrige, j'entre les corrections à l'ordinateur, je le corrige, je relis tout depuis le début, et je passe au chapitre suivant... [Littérateur, Paris]

Sans doute ceux qui ont fait leur éducation avec l'ordinateur, tel ce jeune auteur expliquant qu'il est un frère jumeau de I'Internet et des ordinateurs, ont-ils davantage tendance à se passer de la phase d'écriture manuscrite, mais cela ne signifie pas pour autant que la phase manuscrite devient superflue :

J'écris directement avec l'ordinateur. Je n'ai jamais connu de machine à écrire. Comment je fonctionne: j'écris tout sur l'ordinateur, direct, je retravaille, et retravaille et retravaille, toujours sur l'ordinateur, et ensuite, seulement très longtemps après avoir retravaillé sur ordinateur, j'imprime. Parce que tant que ce n'est pas un objet physique, je peux supprimer des passages entiers. J'ai moins de remords puisque je ne supprime pas les pages. L'objet reste immatériel. Puis j'imprime une première fois, et là je corrige sur papier au stylo. On ne corrige pas les mêmes choses, il faut déjà qu'il y ait un gros travail de correction qui a déjà été fait sur l'ordi. On corrige quand même beaucoup de trucs mais on n'a plus le même regard. C'est un boulot de fourmi, il y a des moments où on s'énerve, on regarde chose par chose. C'est une des choses qui est sympa du boulot sur ordinateur et puis sur papier, c'est deux moments différents, tu ne peux pas faire ce type de travail sur l'ordinateur, tu dois écrire, le voir. Après quand tu as fini, tu 
mets le support papier sur tes genoux, et tu rentres les corrections avec l'ordinateur. Mais là, du coup, tu repenses à chaque modification et tu te demandes si c'est une bonne correction ou pas. Et à la fin, il est bon. Il y a un vrai dialogue entre les supports. Les deux ont pour la correction leurs aspects super intéressants. Je pense que quelqu'un qui a tout écrit à la main manque cela. J'ai fait ça pour mon premier livre, j'avais écrit un chapitre à la main et puis rentré dans l'ordinateur... Tu te reposes toutes les questions quand ça arrive. II y a des allerretour, je pense que c'est vraiment le plus grand truc que la numérisation a changé : un dialogue entre les supports au moment de la correction, pas au moment de l'écriture. II y a une rythmique, une musique qui n'est pas la même qu'on peut retrouver dans le style et dans la manière d'amener son raisonnement à une conclusion ou une autre. [Jeune auteur, Paris]

Ce "dialogue des supports " se concentre sur la phase de correction du premier jet et non sur la conception; mais cela aboutit à une réélaboration assez forte du texte et surtout ces aller-retour font s'interroger notre jeune auteur sur ses choix d'écriture. Certains auteurs ont quant à eux du mal à passer à l'écriture sur ordinateur, parce qu'ils se sont habitués à écrire à la main. Ceux-là évoquent des problèmes de concentration.

Moi j'écris à la main et je lis sur les livres parce que je suis de l'ancienne génération. C'est quelqu'un qui tape pour moi, je ne peux pas taper et rester concentrée. (...) II y a toute une sensation que vous n'avez pas avec l'ordinateur. Donc pour le plaisir, les choses traditionnelles, c'est le papier mais pour aller vite et travailler bien, l'ordinateur c'est fabuleux. Donc avoir les deux c'est magnifique. [Vulgarisation, Paris]

Les fonctions spécifiques de l'ordinateur, comme le couper/coller, les déplacements des textes, la circulation accélérée, les fonctions de recherche sont appréciées par des auteurs:

Je tape, dieu merci j'appartiens à cette génération pour qui ça change juste la vie, de pouvoir couper, copier, coller, corriger, noter des idées sur un doc à part, c'est un gain de temps inestimable. [Romancière, Communication, Paris] 
Mais ces caractéristiques ont aussi des corollaires; il devient difficile (parce que c'est trop facile) à certains de mettre un point final :

L'inconvénient de l'ordinateur, selon moi, c'est son caractère obsessionnel. On peut changer tellement facilement que je peux changer huit fois quelque chose, pour finalement le remettre comme au début. En fait, paradoxalement, l'ordinateur est une vraie perte de temps. A la fin, j'envoie le texte imprimé à l'éditeur. [Romancier, Paris]

Une autre évoque un retour en arrière; l'ordinateur, trop facile, venant presque corrompre la qualité de l'écriture :

Au début, j'écrivais directement sur l'ordi. Mais ce n'est pas sérieux. J'écris sur des carnets de molesquine. C'était moins abouti quand je ne passais pas par le manuscrit. Maintenant, j'écris à la main puis je tape. [Littérature enfantine, Ajaccio]

Certains de nos auteurs, peu nombreux, utilisent exclusivement l'ordinateur pour écrire et ne passent qu'in fine par la phase papier. Ceux-là impriment leur travail quand ils le considèrent comme achevé pour le communiquer, voire ils ne l'impriment pas du tout. Parmi eux, on retrouve l'auteur qui a d'abord été blogueur et qui écrit ses livres comme il écrivait son blog, en en ayant la disponibilité permanente :

Alors moi j'écris dans Google Doc, pour être connecté, pour pouvoir être en contact tout le temps avec mon texte, partout dans le monde et puis ça permet de travailler dessus à plusieurs à distance et en live. [Auteur-blogueur, Paris]

Cet extrait aborde deux aspects importants de la vie du manuscrit, pour lequel cet auteur est sans doute précurseur. D'une part, le manuscrit n'a plus le caractère exclusif qu'il pouvait avoir dans sa version manuscrite, ou même dans sa version hybride, mi-tapu-, mi-manu-scrite; même dans sa phase de conception, il devient indépendant de son support, de son outil de production. D'autre part, le manuscrit est détachable d'un outil spécifique; ce n'est plus un original, mais un texte démultipliable, qui peut être modifié en plusieurs lieux, par plusieurs personnes, éventuellement de manière simultanée.

Ces auteurs qui ne travaillent que sur ordinateur ne laissent pas, ou dans une moindre mesure, de traces de leur travail 
d'écriture et de correction. II n'y a plus guère dans leur cas de manuscrit, de biffures ou d'épluchures, mais seulement plusieurs versions d'un produit fini21.

Difficile donc d'établir pour les auteurs au temps du numérique (comme pour leurs prédécesseurs), malgré la présence généralisée de l'ordinateur, une ligne de conduite unique, un "mode d'emploi " exclusif de la démarche d'écriture ou une procédure technique liée au numérique. Face à l'exigence toujours maintenue que représente "l'idée d'écrire ", la pratique de l'écriture est interpelée par l'irruption des technologies numériques. En effet, les auteurs interrogés n'ont pas un rapport neutre au numérique ; il fait partie de leur quotidien ; il peut-être vécu comme un facilitateur, comme une contrainte formelle, comme une étape dans leur démarche de création, comme un médium indispensable... II leur offre des capacités de stockage étendues, des possibilités d'hybridation des textes, des images, des sources internet..., il leur permet de couper/coller, de revenir en arrière sur les versions antérieures ou les corrections, de comparer automatiquement des textes, de compter les signes, etc.

Le palimpseste, qu'est un manuscrit, est réuni dans un même support immédiatement accessible à celui qui le souhaite (et en a les compétences de base). Le disque dur est par exemple un container aux capacités de stockage infinies; peuvent y figurer des informations potentiellement réinjectées dans le texte, les différentes versions avec les modifications apportées au texte, un stock de photographies exploitables ou inspirantes, des brouillons d'idées, de notes, de commentaires... Bref, l'auteur voit ses capacités d'architecte d'un livre démultipliées, avec une élaboration de plus en plus plastique et un format modulable à l'infini. Le manuscrit est toujours susceptible d'être transformé,

\footnotetext{
${ }^{21}$ Notons qu'à moyen et long terme, si l'on ne trouve aucun moyen de conserver des traces du travail de tels auteurs, cela condamnerait la codicologie (étude des manuscrits) à ne s'intéresser qu'aux textes dont l'écriture précèderait la numérisation. Sans doute existe-t-il aussi des auteurs qui conservent les versions successives des fichiers numériques de leurs œuvres... mais avec quelle durée de conservation et pour quels usages?
} 
d'autant plus que chaque modification est visible et réversible. Bref, avec le stockage sur disque dur l'élaboration du livre s'effectue par un jeu de différences et de versions réécrites dont l'effacement est partie intégrante. L'auteur n'est plus tributaire de la fragilité de l'objet-livre en devenir, de la fixité du papier.

L'ordinateur peut apparaître comme une forme de centralisation singulière d'un travail d'écrivain qui se veut souvent épars et, en sa maturation, chaotique. Le numérique serait pour l'auteur un moyen de captation du flux des idées, du flux de l'écriture pour aboutir à un premier produit fini : le manuscrit dont l'élaboration devient dès lors plus accessible.

\section{Le manuscrit}

Le texte une fois rédigé, vient le temps pour le manuscrit de quitter son auteur pour se transformer en objet-livre. Tout comme la phase de rédaction, sa production peut être transformée par la numérisation.

\section{L'envoi}

Les possibilités de l'outil informatique permettent aux auteurs d'utiliser des logiciels de mise en page, d'utiliser des polices d'écriture qui font du livre un objet répondant à un format uniforme. Cette phase de mise en forme est une phase obligée pour faire du livre un produit de négociation avec un éditeur. Cette mise en forme donne à voir un " résultat " considéré comme acceptable, du moins dans sa forme. On a là une forme de standardisation du produit-livré, médiée par le recours aux technologies numériques: dans leur aspect, avant l'envoi à l'éditeur, les manuscrits se ressemblent donc probablement davantage que dans la phase de la machine à écrire, même si celle-ci avait déjà contribué à leur homogénéisation formelle. Le monopole ou plutôt l'hégémonie de l'outil d'écriture de Microsoft, Word, contribue à ce cadrage, dénoncé ou même refusé par certains :

L'auteur écrit un manuscrit. Notez bien : un manuscrit n'est pas un livre. C'est une liasse de pages de texte écrit ou un fichier texte sur un ordinateur: de nos jours, la plupart des gros édi- 
teurs insistent - sans raison valable - pour recevoir un fichier Microsoft Word 2003.doc, probablement parce que c'est le format le plus couramment utilisé qui permet de mettre des italiques, de souligner, de mettre en gras, de faire des titres. [Science-fiction, Angleterre]

On retrouve ici la même allusion au format textuel et à son importance, déjà évoqués un peu plus haut par l'auteurblogueur qui disait :

Quand je travaille sur Google doc, je suis connecté, je suis dans le Cloud, j'envoie mes mots dans une matrice tout le temps, c'est vivant, il y a un échange constant avec cette matrice. Je déteste écrire dans Word, c'est comme écrire sur du papier, Word c'est mort.

Ce format, Word, inscrit, au moins pour les auteurs qui en maîtrisent les règles, certaines normes dans le manuscrit, et pas seulement des normes formelles, mais aussi des façons d'organiser le texte (par des niveaux de titre) et d'en souligner des éléments (par des soulignés, des gras ou des italiques particuliers). Ce format spécifie aussi les modes d'intervention dans le texte, en établissant la primauté de l'auteur, en en figeant le contenu, à l'image, comme l'explique le blogueur, du papier, et donc d'un texte figé.

On constate aussi, dans la citation précédente, la séparation entre ce que l'auteur considère comme un manuscrit et ce qu'il considèrera, ensuite, comme le produit fini, i.e. le livre. Le manuscrit est destiné à la communication entre l'auteur et son éditeur: d'abord à "l'approche " d'un éditeur potentiel, dont c'est le premier contact avec le texte, puis au travail de celui-ci. II est une étape dans son travail, pas un achèvement. Au moment de l'envoyer à un éditeur, la plupart des auteurs interrogés choisissent le format papier. Certains justifient cela en expliquant qu'ils ne veulent pas qu'un fichier numérique, qui est facile à reproduire et à faire circuler, soit sur un autre disque dur que le leur:

Je ne veux pas que ça voyage. Et en plus, j'ai trop peur que les gens le lisent sur leur écran. [Jeune auteur, Paris]

Certains, parmi ceux qui envoient un fichier numérique plutôt qu'un courrier avec le manuscrit dans sa version en 
papier, se sentent "dépossédés ", comme s'il manquait une phase nécessaire :

En janvier 2010, l'éditeur a pris de mes nouvelles ; je lui ai dit " j'ai terminé [le manuscrit]! ". Je l'ai envoyé par internet. Ça m'a fait une dépossession brutale. La remise physique, c'est mieux... J'avais dématérialisé... j'avais un coup de vague à l'âme. [Policier, Roman policier, Paris]

Pourtant d'autres disent préférer l'envoi par mail sans y voir le moindre inconvénient, comme cette romancière et professionnelle de la communication pour qui cela relève de l'évidence. Les pratiques, selon les auteurs interviewés, sont très différentes. Tout au plus observe-t-on une tendance pour le premier manuscrit plutôt envoyé sous forme papier. C'est d'ailleurs la requête de nombreux éditeurs qui, sur leur site, demandent expressément aux nouveaux auteurs d'envoyer leur manuscrit en version papier. Ensuite, lorsque la relation est nouée, les pratiques diffèrent, chacun fait comme il l'entend, le numérique (l'outil informatique et le réseau) permettant simplement d'offrir à chaque auteur une palette de choix plus importante.

\section{Le manuscrit comme signe de reconnaissance}

Le manuscrit étant le premier contact de l'éditeur et du texte, il est important pour certains auteurs de le soigner "numériquement", afin d'être identifiés immédiatement comme dignes d'intérêt, et de faire en sorte que la lecture ait lieu dans les meilleures conditions possibles.

Ce n'est pas le format d'un livre, c'est plus grand et ce n'est pas très amusant à lire. Et puis, les éditeurs en reçoivent tellement des manuscrits, ils passent leurs journées avec ces trucs dans les mains, qui ne sont pas des objets très sexy. Avec un format papier, ils ouvrent, ils balaient, tu ne peux pas faire ça sur un ordinateur. Si j'ouvre n'importe quel livre, je peux voir très rapidement si c'est bon ou pas. Si c'est bien écrit. Après je ne sais pas encore ce qu'il raconte, mais je sais si c'est bien écrit ou pas. Je crois que c'est parce que l'on est plus habitués, peut-être que cela va changer, mais on est plus habitués à lire des livres et donc l'œil est chez lui. II faut que quand l'éditeur l'ouvre, il sache, il faut que ça l'accroche, il y a un truc de séduction. " [Jeune auteur, Paris] 
Le format papier, contrairement au format numérique, permet à l'auteur de " montrer patte blanche " à l'éditeur, grâce à différents repères (présentations, format, usage -ou abusdes ornements, des polices, reliure...), afin que celui-ci voit que l'auteur de tel ou tel manuscrit n'en est pas à son coup d'essai, qu'il sait comment l'édition et la publication fonctionnent; de ce diagnostic, dépendra la manière dont l'éditeur considérera le texte qu'il a entre les mains et l'attention qu'il lui accordera. Ce qu'il faut faire n'est pas toujours ce que les auteurs auraient envie de faire dès lors qu'ils ont une version finale de leur texte :

Mon premier livre, je l'ai imprimé et je voulais le relier avant de l'envoyer. Mon ami-conseil m'a dit de ne pas le faire relier: chez l'éditeur, ils sont cinq à le relire en même temps. Le premier lit les premières trente pages, puis il les passe au deuxième qui commence à le lire tandis que le premier entame les trente pages suivantes, le deuxième les passe au troisième quand il a fini... ils peuvent lire très vite. [Policier, Roman policier, Paris]

Pour cet auteur, comme pour le suivant, ne pas relier le manuscrit revient donc à envoyer un signe: il connaît les codes.

$50 \%$ des gens qui écrivent des romans mettent leur nom en très grand dessus, et souvent des dessins; ils expliquent que pour eux c'est l'œurre d'une vie et que ça va changer la littérature française, etc. et ça tu le vois. II faut pas non plus que cette version papier ait l'air trop coûteux, ça doit donner l'air que l'auteur a payé 8 euros pour un document de travail, pas de dessin, le titre en gros, le nom et l'adresse en petit comme ça, les éditeurs peuvent le renvoyer vite, recto verso pour que ça coûte moins cher, et puis bien chapitré pour qu'ils voient le plan, mais pas plus. Là, ils ouvrent, et ils voient que je sais comment ça fonctionne. Ce n'est pas trop désagréable à lire non plus, il y a un interligne 1,15 , je rends la chose souple. Ils peuvent le gribouiller, ce n'est pas du tout un livre. Alors qu'il y a des gens qui envoient déjà un livre. [Jeune auteur, Paris]

Certains auteurs font pourtant du manuscrit un objet fini, pour le distribuer à leurs proches, et pour avoir un contrôle total au moins sur la production de ces exemplaires: 
Ce que j'aime bien faire, c'est quand j'ai fini un manuscrit, je le tape à la machine, je l'imprime et je le fais relier, pour ma garde rapprochée, et cela de façon "super chic ", ça oui, ça j'adore... Mais j'en fais simplement cinq ou six exemplaires que je donne à des copains. [Littérateur, Paris]

Si le manuscrit est conçu par les auteurs comme une étape importante, elle demeure une phase intermédiaire, ce qui, éventuellement, peut faire changer le discours différent visà-vis de la numérisation: il ne s'agit plus de numériser le produit fini, comme pour l'e-book, mais simplement de numériser un produit intermédiaire, pour le rendre, peut-être, plus facile à manier, à échanger, à évaluer, à corriger et à conserver. Étant donné ces caractéristiques, notamment la facilitation de l'échange, certains auteurs préfèrent n'envoyer à leur éditeur qu'un manuscrit papier, pour garder le contrôle sur ce que deviendra ce produit non-fini, tandis que d'autres échangent volontiers par mail. Dans tous les cas, le manuscrit est une étape du travail de l'auteur: c'est le point de départ d'une future négociation, à la fois marchande et éditoriale, avec l'éditeur; c'est aussi le plus souvent le premier format qui sera communiqué à des lecteurs, au premier cercle des familiers (la "garde rapprochée " par exemple évoquée plus haut) comme aux éditeurs. Ces deux premières catégories de lecteurs contribuent ainsi à amener l'auteur vers l'édition de son livre, en apportant un regard extérieur, en lui faisant connaître les codes et habitudes, en actualisant sa position de producteur d'ouvrage.

\section{La fabrication du livre}

\section{La communication entre éditeur et auteur}

Une fois entre les mains de l'éditeur, le manuscrit devient un document de travail. De la même manière que l'ordinateur et la plume sont les supports du travail de l'auteur, le manuscrit est le support du travail de l'éditeur. Son rôle vis-àvis de ce manuscrit est important pour les auteurs :

C'est comme si je donnais à un pianiste une partition et qu'il me dise alors là, techniquement, je ne peux pas jouer parce 
que ce n'est pas faisable, et deuxièmement, à l'oreille, ça ne va pas. Un manuscrit, c'est la même chose. Et de la même façon qu'un compositeur, lorsqu'il fait jouer ses partitions, corrige pendant la répétition, parce que ça sonne mal ou que la couleur n'est pas bien répartie, eh bien moi j'attends d'un éditeur qu'il fasse ce travail là, qu'il me dise: "là tu peux peut-être mieux répartir la couleur, l'univers, l'ambiance... " ou alors " c'est très mal écrit ", mais ça, heureusement, personne ne me l'a jamais dit. On doit travailler sur les termes, sur la précision... C'est ça qui est intéressant, j'ai souvent en tête une image, je n'ai pas toujours le bon terme, et pendant le travail avec l'éditeur, les choses se précisent et on trouve ensemble le bon terme, encore plus fort, pour exprimer ce que je ressens... C'est exactement cela que j'attends d'un éditeur. [Littérateur, Paris]

Pour la composition générale, à la première lecture, un éditeur va te dire " ça va " ou "ça ne va pas ". Je pense que sur la composition générale, je n'ai pas de problème. Après, il faut resserrer des boulons, peut-être plus sur des temps de chapitre, des longueurs. Hier soir, j'assistais à un concert, eh bien sur une des parties, qui durait un quart d'heure de vocales, on aurait pu resserrer à sept minutes, c'eût été alors plus percutant, plus efficace... En littérature, c'est pareil. On ressert des chapitres. On enlève tout ce qui n'apporte rien. " [Littérateur, Paris]

Négociation car il n'est pas toujours facile pour les auteurs, de quelque type de livre que ce soit, d'accepter une intervention extérieure sur leur texte, de reconnaître une autorité qui se revendique aussi de la qualité :

Quand j'étais plus jeune, je ne voulais pas qu'on change une virgule. Mais c'était un peu con. Je dois reconnaître qu'en toute simplicité un œil extérieur propose parfois une phrase qui est meilleure. J'ai appris l'humilité. Pour toutes les modifications qu'on me propose, je regarde, et je peux dire oui ou non. Ils m'envoient un fichier papier avec les modifs proposées à la main et je choisis celles que j'accepte. [Romancier, Paris]

Mais négociation relativement douce car, parmi les auteurs rencontrés, cette phase du travail n'est jamais celle qui pose de gros problèmes ou qui suscite les conflits; les auteurs se plaignant plus de l'absence d'un regard attentif, voire critique, de révisions trop peu soignées ou erronées que de l'abus d'intervention de leur éditeur, et là aussi, quel que soit le genre des ouvrages. 
L'auteur a fait de son mieux pour retravailler le manuscrit et qu'il soit le plus fini possible, mais il restera inévitablement des fautes de frappe, des maladresses grammaticales, des problèmes de transition, des incohérences dans le récit, et des passages où l'auteur s'est embourbé parce qu'il écrivait en pilotage automatique. Et là je parle des bons manuscrits, ceux qui sont publiables, écrits par des auteurs reconnus. [...] L'auteur relira le manuscrit revu par le correcteur. Pour des raisons obscures, les assistants de mon éditeur adorent m'envoyer les épreuves à relire la veille de Noël pour le 4 janvier au plus tard! C'est encore mieux quand il y en a deux qui se décident à le faire en même temps. On dirait qu'ils pensent que personne ne m'aime et que j'ai besoin d'occupation pendant les fêtes de fin d'année. Hélas, les relecteurs, ne sont pas télépathes. Parfois, ils ont mal compris certains passages du texte; plus rarement, il y a beaucoup de passages qu'ils ont mal compris. Donc quand l'éditeur reçoit le manuscrit relu et corrigé, il le renvoie à l'auteur pour relecture. L'auteur peut voir les corrections du correcteur, et si nécessaire, ajouter ses propres modifications. II y a en général plusieurs corrections par page, allant des choses les plus triviales [...] à " votre héros est désigné sous le nom de Joe 16 fois et sous celui de Jim 14 fois dans le manuscrit, quel nom est le bon ? " [Science-fiction, Angleterre]

Le livre se conçoit comme porteur d'une potentialité de performance, d'efficacité. Le discours des auteurs oscille entre produit rédactionnel et produit marketing, dont il faut " resserrer les boulons ", pour la qualité de la rédaction autant que pour l'adéquation supposée au marché. Les deux logiques semblent converger vers un produit final qui est autant une " œuvre " qu'un produit marchand, résultat d'une maturation nécessairement collective. II n'est pas figé, il est un objet nomade. Ce nomadisme du manuscrit se satisfait aussi bien d'un format papier que d'un format numérique. Mais, dans les deux cas, bien souvent, le réseau Internet facilite le travail en fluidifiant les communications entre l'auteur et l'éditeur. Cela ne saurait se substituer à l'entrevue physique entre eux, mais chaque rencontre fait l'objet d'échanges en amont sur le manuscrit et sur le statut futur du livre ainsi construit. Cette dynamique de co-construction, à laquelle l'auteur est attaché, est importante car elle se nourrit d'échanges immatériels via 
les réseaux et d'un travail " matériel " sur le manuscrit lors de rencontres auteurs-éditeurs :

N'importe quel éditeur peut être n'importe où dans le monde et communiquer avec vous. C'est la rapidité, l'interactivité, l'instantanéité. C'est ce côté-là qui peut être intéressant, le fait de ne pas avoir à se déplacer, etc. ; mais ça ne veut pas dire qu'il ne faut pas se déplacer. Moi, j'ai besoin de voir la tête qu'a mon éditeur, de manger avec lui, etc. Si Internet coupait cette relation-là, finie l'humanité. [Nutrition, Paris]

\section{Le rôle de l'auteur dans la fabrication}

Internet et le numérique permettent aux auteurs qui le souhaitent, quand l'éditeur l'accepte, d'entrer véritablement dans le processus de fabrication de l'objet fini. Ils peuvent présenter leur texte d'une manière qui "donne une idée " à l'éditeur de ce qu'ils souhaitent qu'il devienne :

On remet d'abord le tirage papier à l'éditeur, ensuite c'est lui qui fait la mise en page (...). Pour le prochain livre que nous faisons, nous avons le droit de mettre 150 tableaux et 70 photos je crois. On peut les présenter de telle ou telle façon, ce que je vais faire donc, c'est les numériser et ensuite tenter de les mettre dans le texte pour qu'ils voient un peu comment je veux les présenter. En fonction de ça, on discutera... Je ne suis pas embêtante parce que ce sont des gens qui connaissent bien leur travail, donc si les écouter c'est mieux, j'écoute! [Vulgarisation, Paris]

Dans certains cas, l'auteur peut être investi dans la production de l'objet fini, le livre, sa maquette, sa couverture, sa présentation; le numérique semble avoir permis, ou du moins renforcé cette possible participation :

J'apporte une importance capitale à l'objet livre. Et j'ai eu la chance de pouvoir choisir chez mes deux derniers éditeurs la mise en page, c'est-à-dire la police, la taille des caractères, l'interlignage, le titre en haut de page ou bas de page, le positionnement, etc. Tout. Et chez mon avant-dernier éditeur, ça a été formidable, j'ai même construit la couverture avec l'éditeur. Pour moi, le plus bel objet, c'est celui-là, en édition originale, avec des rabats. C'était vraiment un beau livre. Ils m'ont même emmené chez l'imprimeur pour voir comment ça se passe. (...) Chez mon nouvel éditeur, tu n'as pas de contrôle sur la couverture, mais sur le reste oui. J'ai tout choisi encore une fois. Je ne 
lâche pas mon texte à l'éditeur, contrairement à certains auteurs, je l'accompagne jusqu'au bout... L'objet livre, je ne m'en fous pas. II y a le texte, et il y a l'objet, les deux comptent pour moi. [Littérateur, Paris]

Beaucoup d'auteurs se plaignent du manque de dialogue avec l'éditeur une fois le manuscrit livré, de la spécialisation des rôles qui les empêche de faire entendre leur avis, et ce, malgré la production en numérique. Cette plainte est d'autant plus notable que, nous l'avons vu, le rôle joué par l'éditeur est important à leurs yeux, indispensable même. Ils souhaitent, le plus possible, travailler "avec " l'éditeur, et non pas seulement " avant " lui :

J'avais demandé à la lectrice d'annoter le livre car je trouvais parfois quelques remarques pertinentes, et même si dans l'ensemble ça ne l'était pas, je voulais une relecture complète. II y avait des suggestions de coupe intéressantes. Elle a dû mettre une annotation toutes les trois pages. En même temps, elle n'avait pas vraiment aimé le livre; on lui a dit de travailler dessus, ce n'est pas facile non plus pour elle. Pour moi ça n'apportait rien ce qu'elle me disait, mais je n'étais pas fermé à l'idée d'écouter des conseils pour l'améliorer. [Premier roman, Paris]

Je me suis un tout petit peu mêlé de la fabrication du livre chez mon éditeur. J'ai fait des suggestions qui n'ont pas toujours été suivis d'effets. Sur les notes, les façons de présenter les choses. Mais ils sont restés sur la défensive. La responsable maquette ne pouvait pas accepter qu'un clampin lui soit préféré. Ça remettait en cause son statut de professionnelle. [Philosophe, Paris]

L'ex-blogueur qui, lui, ne veut travailler qu'en numérique, trouve en face de lui des professionnels qu'il considère comme trop peu aguerris à ces technologies Internet de production et de diffusion des textes :

J'ai un rapport consterné avec le monde de l'édition, avec ceux avec qui j'ai travaillé. Ils n'ont pas tiré d'enseignements de ce qui se passe avec la musique. Pour eux, signer un auteur en numérique, c'est le Far West. II y a bien quelques initiatives par ci par là... mais bon. D'ailleurs ça se voit dans le travail. II faut encore faire beaucoup de chose en papier avec un éditeur, alors que moi j'aime travailler dans Google Doc. Il y a un vrai re- 
fus des éditeurs à se mettre au numérique. [Auteur-blogueur, Paris]

Dans la chaîne de fabrication du livre sont donc à l'œuvre des dynamiques de négociation et de co-fabrication où se mêlent légitimité de l'écrivain, statut de l'éditeur et capacité technique de l'infrastructure éditoriale (maquettistes, correcteurs, PAO, directeur artistique, graphiste...). Si le recours au numérique permet d'assouplir les échanges entre les trois principaux pôles (auteur/éditeur/infrastructure), il peut sembler critique à l'auteur, qui peut se sentir rapidement dépossédé de son manuscrit, le numérique permettant à faible coût un niveau d'intervention élevé.

Le modèle du studio de BD qui se développe pour certaines productions semble être le modèle le plus à même d'atténuer un certain nombre de difficultés liées à la dynamique de co-construction du livre. En effet, dans le cadre de la $\mathrm{BD}$, dont la réalisation rassemble généralement plusieurs catégories d'artistes, certains auteurs et artistes décident de se regrouper dans des studios. Ceux que nous avons rencontrés en ont fait un "hors les murs" qui présente l'avantage de bénéficier de coûts de fonctionnement bien moindres: absence de locaux communs à gérer, pas d'investissement matériels et autres charges associés au regroupement physique de ce type de collectif.

Au-delà des économies de structure, cette organisation collective à distance sous forme de studio permet d'intégrer le monde de l'édition et d'ouvrir les portes de nouvelles maisons en proposant et en fournissant des prestations plus " techniques ". Sont rassemblés, au sein d'un endroit dédié, auteurs, dessinateurs maquettistes, et toutes les compétences impliquées dans le travail de la bande dessinée (comme les lettreurs); cela facilite les démarches auprès d'éditeurs: le studio leur fournit d'abord une prestation technique, puis en profite pour leur proposer des projets plus complets. Si ces studio ne semblent pas être forcément bénéficiaires (seuls les artistes sont rémunérés pour les prestations fournies), ils apparaissent comme des plateformes efficaces de promotion et de prestations et des formes d'organisation mieux à même d'équilibrer les modali- 
tés d'échange entre éditeur et auteur. Ce dernier n'est plus isolé face à la structure éditrice et peut s'appuyer sur un collectif dans la régulation des échanges présidant à l'élaboration d'un livre.

\section{Conclusion intermédiaire : le dialogue des supports}

L'écriture d'un livre n'est pas radicalement transformée, pour la majorité des auteurs, par le passage au numérique. Quelques auteurs considèrent le numérique comme une étape et publient d'abord sous format d'e-book, celui-ci servant de produit d'appel à leur ouvrage. Quelques autres, encore rares eux aussi, ont d'abord écrit un blog avant de penser à réunir leurs billets sous forme de livre. La plupart des auteurs, au moment où ils parlent de la décision "d'écrire un livre " ne parlent pas du format ou de l'objet, mais plutôt du sujet, de la démarche d'écriture, de questionnement, de l'idée - un parcours intellectuel et personnel plutôt qu'un objet tangible. Et, tous, même ceux qui n'y avaient pas pensé au départ, semblent considérer le papier comme un objet plus " noble ", plus "abouti ", plus durable, même celui qui, parmi eux, ne comptait pas voir ses textes publiés sur le format papier.

Je pense que les auteurs auront toujours besoin de versions physiques de leurs œuvres parce que je ne connais pas un auteur qui ne soit pas un amoureux des livres. Du coup, le plaisir de voir son travail dans un objet est incomparable au plaisir de le voir en numérique. Parce que le numérique, il le voit avec son Word. Je crois que les auteurs voudront toujours avoir une version papier, quoiqu'il arrive. [Romancier, Picardie]

Une fois la décision d'écrire prise, nos entretiens font apparaître un véritable dialogue entre supports au moment de l'écriture elle-même. Si certains auteurs écrivent uniquement grâce à l'ordinateur, la plupart écrivent à la fois à la main et sur le clavier et vantent les bénéfices qu'il y a à passer d'un support à l'autre, du papier à l'écran et de l'écran au papier, au moment de corriger, de ré-arranger, de remettre en cause leur travail, de le perfectionner et, enfin, de finaliser un manuscrit qu'ils rendront à l'éditeur. Ils soulignent aussi que les corrections effectuées à la main et 
celles effectuées sur la machine ne sont pas du même ordre. Les brouillons papier peuvent être gardés, tout comme les versions numériques successives d'un fichier texte, mais le fichier numérique peut faire l'objet de manipulation (comme de mettre en évidence les modifications successives ou de récupérer en un seul clic un passage laissé de côté). Les traces d'élaboration du travail d'écriture peuvent donc toujours être conservées, mais elles ne sont pas identiques: les brouillons papier sont moins finis, marqués voire obscurcis par l'écriture manuscrite; les versions des fichiers sont plus lisibles, plus manipulables. Mais les seconds sont attachés à l'ordinateur (ou aux disques de stockage) de leur auteur et donc finalement plus personnels, plus attachés à leur auteur que les versions imprimés ou manuscrites.

Se pose ensuite la question de la modalité de fabrication du livre et de l'élaboration d'un style d'écriture. Le recours au numérique contribue-t-il à standardiser le produit ? Comme l'a montré McLuhan, toute modification de la forme d'un média modifie également son contenu. Le numérique aurait ainsi non seulement une incidence sur l'objet livre mais, plus subtilement, sur l'objet écriture: il existe aujourd'hui des logiciels qui permettent d'écrire des livres; au Japon, par exemple, des romans sont spécifiquement écrits pour convenir à des supports mobiles de type Smartphones. II ne convient néanmoins pas d'opposer papier et numérique mais de s'interroger sur les évolutions du travail classique d'écriture à l'ère du numérique dont les entretiens nous ont montré la complémentarité. Celle-ci se joue dans la maturation de la pensée de l'auteur, dans la capacité de stockage des procédés numériques et dans l'exploitation des données sur Internet lors de la recherche d'informations. Un livre historique peut désormais s'écrire de chez soi en exploitant les encyclopédies en ligne ou les archives numérisées; un livre sur la santé trouve tous les articles médicaux sur les bases de données scientifiques. De même, l'auteur peut désormais faire appel à son éditeur pour exploiter des banques d'images dont il ne dispose pas ou dialoguer directement avec le maquettiste sur un modèle de mise en page approprié à son propos. 
Commence alors un travail qui peut avoir lieu sous forme papier, sous forme numérique, ou les deux, et qui consiste en des allers retours plus ou moins nombreux entre l'éditeur et l'auteur qui, à des degrés divers, selon sa volonté et son rapport avec l'éditeur, peut être impliqué dans la fabrication de l'objet-livre. Dans tous les cas, les nouveaux usages évoqués ici nous amènent à une interrogation approfondie sur la reconfiguration de l'objet livre.

\subsection{L'objet livre reconfiguré}

Le livre (même si c'est sur ordinateur), c'est comme une potion magique, c'est ce rapport à la puissance du livre que j'ai toujours voulu défendre. [Littérature enfantine, Paris]

\section{Le livre " en continu "}

Une des différences majeures entre le papier et le numérique réside dans le fait que, sur le premier de ces supports, l'information est fixée, inscrite, immuable. II peut y avoir de nouvelles versions du livre papier, des rééditions revues et complétées, une version en poche, de club, voire résumée être éditées, mais cela n'empêche pas les anciennes éditions d'exister, d'être lues, consultées et citées. À l'inverse, le numérique permet la continuité, c'est-à-dire la conception du livre comme un processus qui brouille les frontières anciennes entre les étapes de la conception du manuscrit et celles de la fabrication de l'objet fini.

On peut désormais imaginer un livre qui soit sans cesse écrit par son auteur, tout en étant dans les mains de l'éditeur et du lecteur. Un livre remis à jour en direct, actualisé, " continualisé ", comme le sont les dépêches d'agence, lesquelles sont d'abord publiées dans une première version, puis sans cesse revues jusqu'à leur version définitive, qui n'est en fait que l'ultime version avant qu'elle ne soit abandonnée :

Je me suis inspiré d'un principe de l'Associated Press et d'autres agences de presse, qui utilisent ce qu'on appelle le processus "d'écriture directe", qui consiste à alimenter un contenu par l'ajout régulier d'informations, en disant aux éditeurs ce qu'il y a de nouveau dans l'histoire. C'est l'approche 
que j'ai choisie pour ce projet : les commentaires de blog viennent nourrir les chapitres en cours d'écriture qui sont accessibles publiquement. Les chapitres continuent d'évoluer même une fois le livre publié. Mais il est encore vraiment trop tôt pour savoir avec quelle fréquence les versions mises à jour du livre seront publiées, à supposer qu'elles soient publiées. Étant donnés les progrès rapides dans le domaine de l'impression à la demande, il y en aura peut-être plus que je n'imaginais au départ. Dans les trois jours qui ont suivi la publication de $M$... en format PDF sur le site, environ mille cinq cents visiteurs l'ont téléchargé, et bien plus encore ont été voir le sommaire de la version HTML qui comporte des liens actifs vers les ressources du site. Cela a d'ailleurs permis aux gens de repérer des erreurs dans le livre et de m'en avertir, ce dont je leur suis très reconnaissant. [Sciences humaines, États-Unis]

Ainsi, le feed-back des lecteurs peut devenir une partie de l'écriture, si l'auteur, l'éditeur, ou les deux, décident de l'incorporer d'une manière ou d'une autre dans le "produit fini " qui, dès lors, apparaît comme un "produit indéfini ". Notamment, les informations peuvent être vérifiées et complétées par les lecteurs, à la manière de Wikipédia ou en posant un certain nombre de nouvelles questions : comment rémunérer ces contributeurs auxquels on a externalisé, "crowd-sourcé " dans le vocabulaire numérique, l'activité de vérification, auparavant à la seule charge de l'éditeur? Comment choisir les moments où l'on décide de faire une nouvelle édition papier si le livre existe dans les deux formats ? Car en effet, si une version papier existe, et que la version numérique est rendue continue, les deux objets diffèrent de plus en plus jusqu'à ne plus être vraiment les mêmes, puisque différeront le contenu et au moins une partie des auteurs (et de fait ils seront plus nombreux).

Ce format peut également servir de "mise à l'épreuve " du manuscrit par les auteurs, avant d'en venir à la version papier, considérée alors comme plus aboutie parce que (au moins provisoirement) "définitive". Le livre peut aussi par exemple être publié graduellement, à la manière des feuilletons comme ceux que publiait Balzac ou Eugène Sue :

Je mettais en ligne les chapitres du livre dès qu'ils étaient écrits, pour que les gens donnent leurs impressions au fur et à mesure. [Sciences humaines, États-Unis] 
A... a d'abord été publié de 1999 à 2004 sous la forme d'une série de nouvelles, dans le magazine de science-fiction Asimov. Les nouvelles ont pas mal attiré l'attention - cela a atteint un pic au moment où E., qui devient par la suite le chapitre $8 \mathrm{du}$ roman, a été nominé pour le prix Hugo de la meilleure nouvelle en 2005. La situation était plutôt inhabituelle : A... avait obtenu quatre nominations Hugo en tout [...] avant même que le roman ne soit fini ! J'ai décidé de considérer que c'était déjà en soi une réussite personnelle. [Science-fiction, Angleterre]

On trouve ici une idée de format "apprenant " qui est un hybride entre une pratique ancienne tel le feuilleton, et une pratique nouvelle, issue de l'Internet interactif (le Web 2.0), et notamment des Wikis, qui consiste à prendre en considération les réactions, les avis, les corrections et les "validations " des lecteurs pour, d'une part, fournir un texte final qui correspond mieux à leurs attentes et, d'autre part, pour que l'auteur lui-même puisse en bénéficier pour augmenter la qualité de son travail (mesurée en terme d'adéquation avec son projet éditorial); en somme, pour augmenter la satisfaction de chacun des acteurs du dispositif "livre continu ".

\section{Le livre " enrichi "}

Processus nouveau qui fait du livre un tissage en devenir de contributions et d'interactions, le livre enrichi ne suscite pas forcément les mêmes réactions chez tous les auteurs. On retiendra ici les deux positions extrêmes, celle des sympathisants et celle des réfractaires, sachant que l'attitude de la plupart des auteurs est bien plus continue, que les ambivalents sont nombreux, sans compter les indifférents que nos interrogations poussaient à se poser des questions qui, aujourd'hui, leur importent en définitive assez peu.

\section{Les sympathisants}

Dans ce format de livre enrichi, l'auteur explore une piste d'écriture qui permettrait de rendre le lecteur moins "passif " qu'il n'est supposé l'être dans le format papier ou dans la "reproduction numérique analogue au papier". Le lecteur, avec le livre dit reconfiguré, agit directement, en cliquant, en choisissant, en arbitrant, tandis que différents 
sens sont chez lui sollicités. II passerait du statut de "consommateur " à celui d' " usager ", constant interlocuteur de l'auteur, plutôt que récepteur d'une œuvre terminée. La " continuité " dont il est question dans la section précédente pourrait alors être perçue comme une richesse. Cette conception du livre comme un chantier qui ne serait jamais terminé et à laquelle tous s'affaireraient, jusqu'à et y compris dans la lecture, est perçue comme une aubaine par certains auteurs interrogés. C'est bien le numérique qui rend possible ce livre-chantier.

Pour moi, l'objectif de ce projet est de contribuer à transformer les consommateurs passifs de medias en utilisateurs actifs, avec l'idée qu'ils participent à chaque étape du processus, à commencer par ce qu'ils lisent. Donc ce projet, aussi bien le livre que le site Internet, sont sous licence Creative Commons Paternité - Pas d'utilisation commerciale - Partage à l'identique ${ }^{22}$, qui permet à tous de télécharger et copier le livre (dans un but non commercial), et de télécharger gratuitement tout le fichier PDF à partir de mon site Internet. [Sciences humaines, États-Unis]

La richesse de cet ouvrage repose sur la diversité des compétences et des expériences que nous apportons, et je trouve que l'approche du "chantier numérique permanent " n'a fait que mettre en valeur cette richesse. [Directeur d'ouvrage collectif]

Le livre enrichi permettrait d'insérer des liens hypertextes, ainsi que d'autres formats, vidéos, images, audios, hyperliens, jeux, index... au sein même des pages de l'ouvrage. Plusieurs auteurs sont intéressés par ce format, y ont pensé, mais peu l'ont tenté. Cet auteur pour enfant, aux livres nombreux et populaires, explique à la fois être hostile au numérique, à l'ordinateur et à Internet, et avoir rencontré les personnes qui lui ont en fait comprendre l'intérêt, dès lors qu'il ne s'agissait pas seulement de plaquer des illustrations ou des jeux sur un texte tout fait, mais bien d'inventer une autre forme de récit, une autre définition de la lecture :

Les trucs que j'ai vus en numérique où on tourne les pages, je deviens fou. J'en ai téléchargé une gratuite, je ne vais pas

22 Voir la présentation des licences Creative Commons dans l'encadré c). 
payer en plus pour ça... il nous la montre. C'est honteux ça. Ça c'est on prend le gamin, on le met dans son lit, on lui dit là ça fait du bruit, là ça fait ça, n'oublie pas de le recharger pour demain et bonne nuit. C'est honteux ce eBook, cette espèce de duplication... c'est de la technique... Au lieu de tourner les pages comme ça, tu les tournes comme ça et là c'est l'écran qui tourne les pages, quelle révolution! [Littérature enfantine, Paris]

La plupart des auteurs y pensent comme à un livre enrichi dès les premières phases de la démarche d'écriture, c'est-àdire qu'il faudrait, selon eux, prévoir la conception d'un autre objet que ce qu'on entend habituellement par "livre ", et donc d'autres pratiques, comme peut-être un autre rythme, un autre phrasé, une autre structuration. Certains, en particulier les auteurs littéraires, envisagent une rupture de la continuité textuelle, de sa linéarité, tout en soulignant l'énorme coût en temps de travail d'un tel récit (qui suppose des voies multiples, entrelacées, divergentes). En même temps, ceux-là se demandent s'il s'agira encore de livre et donc d' "écriture " au sens où l'on l'entendait jusque-là :

Ah ça, ça m'intéresse beaucoup, oui. Ça peut être une forme de créativité du siècle qui vient. Ça m'intéresserait d'en faire un. Je ne lis pas de livre numérique mais ça, oui, ça me tenterait. J'aurais d'ailleurs plus de facilité à l'écrire qu'à le lire. Je pense qu'il y a moyen de trouver des liens pertinents, qui s'intègrent dans l'œuvre. En revanche, un texte que j'ai déjà publié pour le papier, ça c'est intouchable. Si on écrit un livre enrichi, il faut le penser comme tel dès le début. (...) Je pense que ça peut être des objets d'arts magnifiques, spectaculaires, mais je ne pense pas vraiment que ce sera des livres. Mais, il y a plein de livres dont je pense que ce n'est pas de la littérature, et qu'ils font du mal à la littérature... [Romancier, Paris]

D'autres, et notamment l'ex-blogueur, qui ont essayé ce format, sont plus réticents.

Au départ on avait l'idée de faire un livre enrichi, et puis finalement, c'est une fausse bonne idée. Pour moi c'est un peu le digipack du $\mathrm{CD}$ : un plus produit pour vendre plus cher, même si je suis sûr que l'on verra de très belles choses. Mais pour moi l'écriture ce sont les mots. [Auteur-blogueur, Paris]

Si "l'écriture ce sont les mots", il n'est en effet plus question de livre enrichi, qui suppose de transformer le livre en 
site web, ou d'ajouter du web au livre. Deux grandes tendances sont explorées, parmi ceux qui pensent les deux formes comme complémentaires et non substituables ou cumulables: les nouveaux formats peuvent venir "compléter " un livre, en donnant de plus amples détails à propos d'un passage, d'une expression, d'un personnage, le lien URL remplaçant alors en quelque-sorte la "note de bas de page "; ou bien les formats pourraient être étroitement mêlés, constituant alors un nouveau "genre" d'objets qui, véritablement, sera multi-facette.

Je souhaite que $M$... fasse l'objet d'un processus continu et itératif, avec des mises à jour sur le site Internet et, à terme, des nouvelles versions du livre lui-même. Cela a vraiment du sens que ce soit un livre numérique parce que les gens peuvent suivre des liens vers les ressources du site web quand ils souhaitent en savoir plus sur un aspect particulier. Je voudrais que M... soit un projet multi-facette, et j'espère pouvoir dans les prochaines années mener des expériences dans beaucoup de formats et styles de médias différents grâce aux idées qu'il aura permis de générer. [Sciences humaines, États-Unis]

Certains auteurs, sans nécessairement en avoir fait l'expérience, ni même avoir nécessairement l'intention d'explorer cette voie, ne sont pas pour autant réfractaires au livre enrichi. Ils y voient l'idée d'un changement des équilibres en place, avec l'apparition d'un nouvel objet, hybride et d'une nouvelle idée de la lecture. Ils imaginent des scénarii futurs sans s'en mêler: ils restent attachés au papier, mais ne déplorent pas l'idée du numérique.

Le passage papier au numérique n'est pas pour demain pour moi. Même mes gosses les plus jeunes sont en lecture papier. Ils lisent beaucoup sur Internet, mais le livre en ligne, c'est une autre conception, ce n'est plus la lecture comme on la connaît. Ce sera peut-être pour des générations qui n'auront jamais connu le papier. Peut-être que le livre numérique avec le mot qui apparaît en bleu, on peut cliquer dessus et ça nous renvoie vers autre chose, ça peut être intéressant... C'est une lecture plus interactive, moins linéaire. Avec les tablettes, peut-être que les bibliothèques vont disparaître. [Médecin, Roman policier, Paris]

Le livre enrichi se conçoit comme un élément venant irriguer le livre classique. II vient en appui à un argumentaire, il il- 
lustre un point précis sans pour autant se substituer à la trame scripturale du livre. Pour d'autres auteurs, il permet un esthétisme interactif et coloré qui peut avoir des vertus d'appel (un produit attractif) mais, pour ceux-là, en aucun cas l'esthétisme ne peut remplacer le processus de lecture.

La publication numérique pour moi ça ne change pas grandchose, en tout cas pas dans l'écriture. Sauf peut-être pour mon prochain livre, ça ne change pas grand-chose à la façon dont moi je l'écris mais ça peut rendre les choses plus agréables pour le lecteur. C'est juste que ça permettra des choses que le papier ne permet pas. II sera question du jeu d'échec; dedans il y aura des diagrammes et dans le texte il y aura écrit "cavalier e4 " et si c'est en numérique on pourra faire en sorte qu'en même temps que le lecteur lit, on voit la pièce se déplacer. [Premier roman, Paris]

\section{Les réfractaires}

D'autres auteurs sont, quant à eux, réfractaires à l'idée du livre enrichi, aussi susceptible soit-il d'ouvrir de nouveaux horizons à la création. Selon eux, la lecture n'est faite que de lecture. Cette richesse que les tenants du livre numérique revendiquent en raison des possibilités démultipliées qu'elle offre dans les manières d'entrer dans le texte, de le parcourir, de l'illustrer, de le compléter, apparaît à ces réfractaires comme une distraction qui défigure leur vision de la lecture. Au papier correspond le papier, à l'élaboration du livre correspond une dynamique de retrait du monde et de ses formats perturbateurs. Ainsi le livre enrichi viendrait troubler leur attente d'un geste créatif libre de toute contingence technique.

Hors de question pour moi! Je pense que c'est justement ce qui fait du mal à la lecture, que les gens ont leur ordinateur, leur téléphone qui sonne ; ils ouvrent une fenêtre, et puis une autre, ils n'arrivent pas à s'asseoir quelque part pour lire. S'il y a un endroit où la numérisation menace plus qu'elle n'ajoute quelque chose, c'est bien dans la lecture comme moment où on se coupe du monde et on suit le raisonnement, c'est très menacé par la génération zapping aux supports multiples et simultanés. Guy Debord appelait ça des "nuisances ", je pense aussi qu'il faut s'en libérer à tout prix. [Jeune auteur, Paris] 
On notera que le rejet du numérique est ici le fait d'une conviction et d'une réflexion mais ne concerne en rien une "fracture numérique " c'est-à-dire une forme d'exclusion de l'individu par l'usage du numérique et des nouvelles technologiques (dans la mesure où notre auteur est parfaitement aguerri à l'internet). La référence à Guy Debord montre bien le choix d'une posture presque politique, en lien avec une distanciation face à une société spectaculaire et marchande, en continuité finalement avec la vision romantique de l'écrivain et de l'œuvre littéraire. On est loin d'un déficit d'acculturation technique mais plutôt dans une conception de " pureté " de la pratique d'écrivain qui revendique plus qu'il ne subit une distanciation vis-à-vis des processus techniques. Le retour à l'objet-livre en tant que tel est notable et structure par son statut le champ littéraire en fixant les frontières de ce qui "fait " livre et de ce qui ne l'est pas: la matérialité du livre papier peut, dans ce cas, aider à re-stabiliser les frontières.

Ces deux attitudes, sympathisants ou réfractaires, auxquelles il faudrait ajouter les ambivalents et les indifférents, ne sont finalement pas des standards figés. Elles mettent plutôt en lumière des écarts de pratiques constitutifs du rapport au livre et à l'écriture, écarts qui concernent aussi les capacités d'innovation du champ littéraire et son adaptabilité au numérique ; l'irruption de l'e-book constitue, on va le voir, un exemple caractéristique de cette dynamique.

\section{L'e-book}

d) Le livre numérique : définition juridique

Jusqu'à récemment, le livre numérique n'avait pas encore été défini en droit. C'est désormais chose faite depuis la loi du 26 mai 2011 relative au prix du livre numérique. La loi se contente toutefois de définir le seul livre dit " homothétique ", c'est-à-dire le livre numérique "à la fois commercialisé sous la forme numérique et publié sous forme imprimée " ou le livre qui "par son contenu et sa composition " est "susceptible d'être imprimé. "Cette définition admet toutefois, dans la mesure où ceuxci sont accessoires, que le livre comporte certains enrichissements propres à l'édition numérique tels qu'un moteur de recherche associé ou "des ajouts de textes ou de données 
relevant de genres différents, notamment sons, musiques, images animées ou fixes, limités en nombre et en importance, complémentaires du livre et destinés à en faciliter la compréhension ". Il est à craindre que cette définition ne soit obsolète dans un futur proche, de par son caractère restrictif : le livre numérique augmenté ou hyper-livre, véritable œuvre multimédia n'ayant pas vocation à être imprimée, n'est en effet pas pris en compte par la définition de la loi du 26 mai 2011.

Au-delà des éléments accessoires relevant de l'édition numérique, la loi nouvelle indique que les livres numériques homothétique doivent être des "œuvres de l'esprit créées par un ou plusieurs auteurs ", reprenant en cela la définition fiscale du livre papier et sa référence au droit de la propriété intellectuelle.

Qu'ils s'intéressent au e-book ou non, les auteurs s'entendent pour dire que ce support de lecture n'est pas, ou ne devrait pas être, à proprement parler une reproduction des pages du livre physique: les codes diffèrent, la langue, l'écriture, ne peuvent être similaires, parce que la logique même de la page ne s'arrête pas au seul texte. II ne s'agit pas seulement, comme le dit cet auteur, de sémantique et d'information :

Pour toucher un large public, les livres numériques devront offrir les mêmes avantages de lecture qu'une édition papier (dead tree edition ${ }^{23}$ ) : une utilisation adéquate de la police de caractère, des critères adaptés pour la mise en page, des fonctions esthétiques, tout cela doit en faire partie. [...] Si vous pensez que les livres numériques sont une histoire de texte uniquement et non de mise en page, je vous incite à aller regarder du côté de l'Internet; à l'origine, le web était davantage porté sur la sémantique et l'échange d'information, et ne se souciait pas de la mise en page et des illustrations, mais aujourd'hui vous ne trouverez pas beaucoup de graphistes qui approuvent cette thèse. En effet, de nos jours, même un site basique de textes comme mon blog a besoin d'esthétique. Donc n'oubliez pas trop vite les vieilles leçons ! [Science-fiction, Angleterre]

${ }^{23}$ L'auteur utilise le terme de " dead tree édition " ou branche morte de l'édition pour désigner la version papier d'un livre qui existe en ligne. 
Ainsi, la transposition du papier vers le numérique ne conviendrait pas à tous les genres littéraires, ou tous les types d'ouvrages. L'e-book nécessite en effet des dispositifs spécifiques - tablette, liseuse, écran - qui changent la nature de l'expérience de la lecture et de l'objet-livre. Celui-ci, même s'il contient exactement le même texte que le format papier, ne correspond plus à la même expérience du lecteur-usager. Il est un fichier, téléchargeable, affiché en deux dimensions, dont on ne perçoit pas l'épaisseur, l'odeur, le toucher, la longueur. L'esthétique elle-même du livre s'en voit modifiée et l'objet de sensation (odeur, épaisseur, toucher) devient un support d'une autre expérience. Pour certains auteurs qui en sont familiers, l'e-book rend l'expérience de lecture plus désinvolte, moins formelle (casual dit notre auteur américain), plus transitoire dans une dynamique de loisir :

Je télécharge pour mon Kindle surtout des livres légers, de divertissement; c'est un peu comme ces films que je regarde une fois puis que j'oublie. Un livre physique, lui, est davantage comme un DVD, quelque chose que j'ai envie de garder et voir et revoir. Les achats que j'ai effectués pour mon Kindle sont l'équivalent des livres que j'achetais dans les points presse des aéroports, des livres à suspens, des livres policiers et des romans de gare que je laissais parfois, après les avoir lus, dans les hôtels ou dans les pochettes au dos des fauteuils d'avion. Une fois que je me suis habitué à lire des livres numériques, j'ai commencé à faire quelque chose qui aurait été complètement improbable à l'ère analogique: acheter des livres qui n'étaient disponibles que dans la version papier relié [donc chère]. Si j'ai commencé à faire cela, c'est parce que je trouvais que dix dollars était un bon prix, et, depuis, mon budget d'achats de livres a grimpé en flèche. [Sciences humaines, États-Unis]

Le renversement est intéressant : le livre physique rejoint le DVD, tandis que le livre numérique, même téléchargé, serait de l'ordre du streaming. Mais surtout l'expérience de cet auteur montre bien que le livre papier a des statuts différents : celui qu'on garde et dont la possession a un prix, celui qu'on abandonne dans les hôtels ou les gares, comme un magazine, celui dont le prix est un déterminant critique de l'achat... On peut supposer que l'e.book vient s'insérer 
dans ces différents statuts et n'entre pas en concurrence égale avec chacun d'entre eux.

Ces différentes approches de l'e-book, les appréhensions, les idées créatrices et les enthousiasmes qu'il suscite sont des éléments non négligeables pour l'auteur. La portabilité et le fait de pouvoir stocker une quantité astronomique de volumes sur un dispositif relativement petit, comme ses capacités innovantes, sont certes des avantages. Ils sont néanmoins contrebalancés par les contraintes juridiques et techniques qui mettent en question le statut de "diffuseur de la connaissance" du livre, tels les DRMs ${ }^{24}$ ou la non interopérabilité des plateformes. Ces dispositifs peuvent alimenter le statut transitoire de l'expérience de lecture, en diminuant la sensation du lecteur de s'être effectivement approprié un livre, de l'avoir fait sien.

II y a du plus et du moins; du plus dans les usages, parce que le lecteur crée des situations de lecture différentes: les liseuses ou e-readers sont légers, ils tiennent dans la main, on peut les lire avec une seule main et passer les pages. Je me suis retrouvé en train de lire alors que mes enfants prenaient leur bain! Des usages se créent, c'est une tendance lourde. C'est particulièrement adapté pour tout ce qui est pédagogique; je pense que les tablettes vont prendre une part très forte sur le marché du livre de loisir et pédagogique ; ensuite, sur la littérature générale, d'après mon expérience personnelle, certaines écritures sont plus adaptées que d'autres à la lecture numérique; tout ce qui est thriller ou science-fiction, par exemple, se lit très bien. Ce qui est plus élaboré en termes de style se lit moins bien. [Romancier, Picardie]

Le principal avantage [du livre numérique] est certainement la portabilité : c'est génial d'avoir avec moi, où que je sois, tout ce que je pourrais avoir envie de lire. [Sciences humaines, ÉtatsUnis]

24 Digital Right Management, il s'agit des " Mesures Techniques de Protection " qui visent à contrôler l'accès aux œuvres numériques et à empêcher d'en faire des copies; elles peuvent limiter sa lecture à un format particulier, empêchant ainsi de fait de lire l'ouvrage sur différents supports, quand bien même le lecteur en a acquis les droits (voir infra 3.3, L'éditeur et le numérique). 
Pour les livres qui relèvent en particulier de la connaissance, de l'apprentissage, de l'essai ou de l'académique, même si le statut de l'e-book reste en devenir, un des nombreux avantages relevés par les auteurs est qu'il permettrait notamment une mobilisation importante des références et des ressources écrites, réduirait les craintes référentielles et permettrait une plus grande précision des sources.

Pour moi, lire un roman sur le numérique n'a aucun avantage. En revanche, j'aime bien pour travailler, notamment pour retrouver des passages. Tu fais "contrôle F", et tu trouves. Je le fais souvent avec Proust par exemple. Ça, je trouve que c'est très bien. J'aimerais bien avoir Muray en format numérique... Je me souviens de certaines phrases, certains moments, que j'ai parfois besoin de retrouver... Mais en même temps, une recherche manuelle, c'est aussi bien, parce que tu retrouves des passages dont tu ne te souvenais pas. Pour la recherche universitaire, là, je me dis que ça doit être très utile. [Romancier, Paris]

L'e-book est ici envisagé comme un support de mémoire, un référencement pour soi de passages particulièrement appréciés propres à telle œuvre, la possibilité de mettre en évidence aisément telle citation. II devient une aide précieuse dans la genèse de l'œuvre à venir, en même temps qu'il autorise une forme de navigation au sein du livre conçu comme un objet ré-appropriable en fonction des formes d'intervention techniques du lecteur. Si l'on peut parler d'une expérience nouvelle propre à l'e-book, celle-ci concerne aussi bien l'auteur que le lecteur. L'e-book, sa conception, ses capacités, sa circulation, son économie, tout comme ses contraintes juridiques et ses standards font l'objet d'importantes évolutions qui empêchent encore d'en cerner les exacts contours.

Par ailleurs, certains auteurs regrettent que l'éditeur n'implique pas davantage l'auteur dans la publication de la version e-book de son œuvre, comme il le fait dans certains cas pour la version papier. L'auteur est alors confronté à une désappropriation, voire un manque de contrôle qu'il n'apprécie guère. L'objet immatériel lui échappe plus encore que ne lui échappait déjà l'objet physique, surtout dans la 
phase de diffusion, pendant laquelle habituellement l'auteur est rendu présent.

Les auteurs ne sont au courant de rien en ce qui concerne les éditions numériques de leurs livres. C'est bizarre mais c'est comme cela. Quand l'édition reliée est publiée, j'en entends beaucoup parler: critiques littéraires, lourdes caisses d'exemplaires d'auteur à récupérer, et ainsi de suite (avec, si j'ai de la chance, une soirée de lancement). Quand l'édition brochée est lancée, idem. Mais les livres numériques sortent par la porte de derrière, sans aucune publicité, ni considération, et sans exemplaire numérique en complément pour l'auteur non plus. C'est vraiment quelque chose auquel on pense dans un second temps, même en 2009/2010. Pour beaucoup de livres, jusqu'à l'an dernier, il n'y avait même pas d'édition numérique du tout. [Science-fiction, Angleterre]

\section{Conclusion intermédiaire : Vers un livre numérique}

Le numérique a permis à certains auteurs de "s'entretenir " avec les lecteurs, et donc de faire du lecteur un usager qui agit, exprime ses besoins, ses remarques, éventuellement suggère des corrections. Le livre numérique peut être publié dans une première version, puis corrigé en continu en fonction de ce qu'en disent les commentateurs, comme un roman feuilleton où, à chaque nouveau chapitre, l'auteur recevrait un courrier abondant dont il tiendrait compte pour les chapitres futurs. Dans cette conception de "livre continu ", le format numérique paraît demeurer une étape intermédiaire, comme un "manuscrit public", dont l'aboutissement serait le papier. C'est sur celui-ci que serait publiée une version cette fois définitive du texte. Ainsi, le lecteur jouerait un rôle proche de celui que joue l'éditeur dans l'industrie traditionnelle: il reçoit le livre continu comme un manuscrit qu'il corrige, en échangeant avec l'auteur.

Nos entretiens montrent un véritable intérêt de la part des auteurs pour les nouveaux formats permis par le numérique, bien qu'ils consultent eux-mêmes assez peu ce nouveau type de "livres enrichis". Parmi eux, certains considèrent néanmoins que le livre enrichi, contrairement au livre continu, n'est adapté que pour certains types de texte, par 
exemple des romans policiers, et qu'il constitue en fait un nouveau type de produit, absolument différent de ce qu'on appelle "livre". Ainsi, ne se sentent-ils pas toujours capables de produire ces nouveaux formats, de la même manière que si l'on demandait à un peintre traditionnel de produire un dessin-animé pour Walt Disney: il y risquerait son savoir-faire, considérant son métier trop différent du savoir-faire et du métier d'un producteur de dessins animés. On peut donc facilement imaginer que le livre enrichi, encore assez rare, aboutira à une nouvelle forme d'expression et surtout d'usage des techniques.

\subsection{Internet comme lieu d'apprentissage}

Comment les auteurs habitent-ils l'espace-temps Internet? Qu'est-ce que le "réseau des réseaux " leur permet en termes d'échanges avec d'autres auteurs, d'autres textes, avec les lecteurs, et comment utilisent-ils la Toile pour se documenter? Dans cette troisième et dernière partie de ce premier chapitre consacré à l'écriture au temps du numérique, le terme "numérique" prend une acception différente: nous nous intéresserons ici au "numérique connecté ", au réseau Internet, et non plus aux outils informatiques d'écriture qui sont souvent, on l'a vu, synonymes d'une démarche qui reste assez solitaire. On se propose ici de décrire comment la "mise-en-réseau" de certains aspects de la démarche d'écriture influe le travail des auteurs que nous avons interrogés.

\section{L'auteur en réseau}

A l'instar de MySpace pour la musique, il semblerait que certains sites ou blogs permettent aux auteurs de faciliter leurs expérimentations. Ils s'y familiarisent avec de nouveaux formats, et surtout, pour des auteurs peu expérimentés, qui n'auraient pas connu la publication à l'ère " proto-numérique ", et qui ont soudain accès à un formidable outil de diffusion de leurs textes. Ce fut notamment le cas de l'auteur-blogueur dont les billets ont eu un succès tel, que ce sont les éditeurs qui sont venus vers lui, et non l'inverse. 
Internet permet également à certains auteurs d'assurer leur propre promotion auprès de publics particuliers, autrefois difficiles à atteindre, via des sites spécialisés. Pour la vente des livres papier, Internet peut également servir de vitrine, où l'auteur "poste " des résumés, des extraits, des notes, et il peut lui-même constituer sa propre plateforme de vente, s'il souhaite se passer d'éditeur. Tel Marc-Edouard Nabe pour son dernier roman, avec ce qu'il nomme l' "antiédition " 25 ou qu'exprime, d'une autre manière, notre auteur picard :

Je pense qu'on aura avec le numérique probablement une multiplication de nouvelles formes d'édition, de gens qui vont se lancer en direct, de gens qui vont essayer de s'auto-éditer. Donc il est possible que ça bouscule le paysage de la chaîne de valeur actuelle qui est éditeur - diffuseur - distributeur. Et qu'on ait aussi des boucles du type l'auteur fait connaître son $œ u v r e$ en numérique et la distribue en physique. On lui passe commande en numérique et hop il en envoie 100 en physique.. [Romancier, Picardie]

Internet permet également des échanges entre les auteurs publiés et non publiés. Les auteurs en devenir, qui avant n'avaient pas d'autre moyen que d'envoyer une œuvre "finie " à des éditeurs potentiels, peuvent recevoir des avis, par exemple, sur leur style d'écriture au moyen d'un échantillon beaucoup plus réduit, publié sur la Toile.

[Ce site pour jeunes auteurs que j'anime] n'a pas vraiment un rapport avec mon activité d'écrivain. Ce qui est amusant, c'est de faire écrire les gens : moi, je n'y écris pas. Quand on écrit, il y a plein de gens qui disent " ah, moi aussi, j'écris ; tu ne connaîtrais pas un éditeur? Et moi je dis : ah bon ? Super, écrismoi quelque chose, alors! Écris-moi une chronique ! C'est gratuit, je ne paie jamais personne. Mais c'est intéressant. [Jeune auteur, Paris]

La solitude du créateur, souvent évoquée par nos auteurs, peut ainsi, sinon être évitée, du moins elle peut être inter- 
rompue provisoirement pour permettre d'échanger sur des pratiques et des soucis communs.

\section{Un outil de recherche et un "baromètre " des temps}

Cela va peut-être de soi : Internet sert aux auteurs d'outil de recherche. Ceux qui, autrefois, étaient familiers des bibliothèques, prisonniers des heures d'ouverture, de la disponibilité de certains ouvrages, etc., peuvent à présent dénicher de nombreuses informations qui servent de fondement à leur travail. Ce qui facilite leur démarche de recherche peut poser aussi des défis propres, liés à l'immédiateté de consultation et de copie de l'outil Internet. Cela a d'ailleurs été reproché à des auteurs en vue ${ }^{26}$, écrivain, journaliste, politique...

Pour trouver des infos, bien sûr... ça ne change pas la manière que j'ai de mettre en scène. Mais je me documente beaucoup sur Internet, ça c'est sûr. J'essaie bien de croiser les sources. C'est très important pour moi de dire la vérité autant que possible, d'être honnête [Romancier, Paris]

Je peux commencer à travailler à 7 heures le matin, chez moi, rien que d'un point de vue matériel, c'est fou. Avant il fallait se lever à 6 heures du matin, aller à la bibliothèque, attendre que ça ouvre... Rien que d'un point de vue matériel, c'est bien. Et puis aujourd'hui ce qui est dramatique c'est que les bibliothèques n'ont plus un rond, elles ne peuvent plus acheter de revues récentes, donc le seul moyen d'avoir des articles intéressants aujourd'hui c'est le net. C'est plus qu'un outil de travail, c'est un couteau suisse, c'est vraiment formidable ! Je ne conçois pas dans ma vie d'aujourd'hui de me passer du net. Quand on part en vacances avec Jean-François (son mari), on est obligé de se battre pour savoir s'il y aura internet dans les locations, on fait tout avec ! Lire le journal le matin, regarder le journal télévisé, une émission en replay... [Vulgarisation, Paris]

26 Pour ne citer que des affaires récentes, c'est le cas de Michel Houellebecq accusé, à l'occasion de la sortie de son roman La Carte et le territoire, d'avoir " pillé "Wikipédia ou du journaliste et directeur du Magazine littéraire Joseph Macé-Scaron, convaincu d'avoir plagié dans son dernier ouvrage l'auteur américain Bill Bryson. 
Pour moi Internet est un outil, une source d'information remarquable, une source d'information médicale. Pour faire un bouquin, ça m'aide énormément, je peux avoir des raccourcis et avoir accès à des informations que je n'aurais jamais eues autrement. Dans le passé il aurait fallu être abonné à des tas de revues pour avoir ces informations-là. C'est effectivement une révolution. Hormis ça, c'est tout. [Nutrition, Paris]

Enfin, Internet permet aux écrivains de trouver des informations à propos de ce qui "préoccupe " les publics, un écho de l'opinion publique parmi d'autres. Sur les forums, ils peuvent voir les sujets qui plaisent, ce qu'on en dit, les tenants des débats, les postures originales. Certains auteurs disent s'en servir comme un "baromètre " de l'époque: un outil capable de mieux comprendre ce à propos de quoi on écrit, et ceux par qui on sera lu.

[Internet et son site pour jeunes auteurs] c'est un bon baromètre pour voir comment va la jeunesse en France. Je vois les sujets qui les intéressent, je les vois réagir... Quelqu'un qui visiterait depuis le début et lirait tout ce qu'il y a dans le site, aurait un bon aperçu de comment marche la jeunesse en France, voilà où ils ne sont pas d'accord, tous d'accord... c'est un bon aperçu des sujets, il y en a toujours qui reviennent. Donc c'est très intéressant de voir ça ; et puis forcément quand tu écris à propos des jeunes, ça te permet de dire des choses qui tapent dans le mille, quand tu fais de la recherche, ça te permet de mieux comprendre le monde dans lequel tu es. [Jeune auteur, Paris]

\section{Internet " facilitateur"}

Comme on l'avait anticipé dans la partie précédente, être en réseau est pour l'auteur une façon de fluidifier les communications avec son éditeur et ses publics. Dans cette troisième partie, il a été question de la façon dont le réseau Internet est imaginé, traité, mobilisé par les auteurs en tant que " facilitateur".

Formidable lieu d'apprentissage, le réseau des réseaux permet, d'une part, d'augmenter la puissance ou l'efficacité d'une démarche ou d'outils auparavant sujets à des contraintes bien plus lourdes: c'est le cas de son utilisation en tant qu'outil de recherche, universel, immédiat et disponible 
en permanence, pervasif au point qu'il faudrait parfois se forcer à s'en détacher, au risque de distraction, voire d'attraction, excessive.

D'autre part, Internet ouvre la voie à des expérimentations à plusieurs titres, notamment en termes de facilitation de la qualité et de la quantité des échanges avec les autres maillons de la chaîne de valeur: les canaux de promotion, les échanges avec les publics et, de façon intéressante, avec les potentiels auteurs de demain qui cherchent leur voie sur la toile, se sentent enrichis et reconfigurés par le réseau.

\section{Conclusion}

Le numérique fluidifie la communication entre l'auteur et l'éditeur dans sa forme: l'auteur, après avoir livré son manuscrit, peut l'échanger plus rapidement et s'impliquer éventuellement dans la fabrication de l'objet-livre. Néanmoins, cela reste encore imparfait: aux yeux de beaucoup d'auteurs, on constate un regret. En effet, la possibilité de communiquer plus rapidement avec l'éditeur n'affecte pas forcément la qualité du contenu de l'échange.

Dans le cas de l'e-book, la "fabrication " du livre pourra coexister avec l'écriture. Pour les auteurs, cela suppose que la communication entre éditeur et auteur devienne plus fluide, l'éditeur ne devant ignorer l'auteur à chaque étape du processus de fabrication. Potentiellement, avec le numérique, les auteurs d'e-book attendent un rapprochement avec leurs éditeurs, des interactions plus soutenues. Ils se veulent associés d'emblée à la fabrication de l'objet-livre dont ils se sentaient trop souvent tenus à l'écart.

On constate donc que le régime d'interactivité entre auteur et éditeur varie grandement en fonction du support envisagé et n'a pas encore trouvé de cohérence propre. On est davantage en présence de régimes distincts dont la logique relationnelle varie grandement, venant ainsi influencer le mode d'intervention des acteurs sur le texte même. Le texte est au cœur des évolutions numériques actuelles: si son élaboration reste l'apanage de l'auteur, son intégrité n'est plus figée. Elle tend à évoluer, à être appropriable et redéfi- 
nissable via différents formats. D'où l'interrogation récurrente de certains auteurs sur la notion de style et de définition de l'œuvre littéraire: l'e-book n'est-il pas davantage adapté aux livres pratiques plutôt qu'à la réception du texte romanesque et littéraire ? Le livre numérique est-il fait pour les romans policiers ou pour les livres de travail? Comment lit-on sur ces supports ? Pour certains, il y aurait une évolution intéressante qui fonderait des évolutions littéraires, non plus du fait d'une recherche sur le style, comme dans le cas du nouveau roman par exemple, mais bien d'une recherche sur le format qui déterminerait le contenu même du livre. II ne faudrait donc plus parler de l'auteur au sens strict mais plutôt de régimes d'auteurs selon le format sur lequel ceux-ci souhaiteraient travailler. L'unité du texte serait donc amplement influencée par la référence au format technologique. Cette perspective déplace les lignes de force du champ littéraire et plus particulièrement de la technologie qui, certes, a toujours joué un rôle dans la fabrication des livres imprimés mais occuperait ici une place centrale qui, selon les auteurs, reste encore à définir. 


\section{Chapitre ᄅ :}

\section{Internet dans la diffusion, la diffusion hors Internet}

S'il y a bien un domaine de la carrière d'auteur (entendu, au sens sociologique du terme, comme l'ensemble des étapes, non nécessairement chronologiques, par lesquelles l'auteur passe pour se réaliser comme tel) affecté par le développement de I'Internet et des outils de communication numérique, c'est bien la diffusion qui apparaît comme la phase de l'écriture la plus immédiatement et la plus systématiquement touchée, et ce quels que soient le degré d'adhésion, le niveau de présence et de familiarité de l'auteur avec ces nouveaux modes d'expression. Par diffusion, nous entendons non seulement la mise à disposition des œuvres, mais aussi les différents dispositifs mis en $œ u v r e$ pour les faire connaitre de leurs potentiels lecteurs, le travail d'animation qui les fait vivre, leur donne une existence publique, les discussions et débats qu'ils provoquent. On s'intéresse donc à la phase où le livre se détache de son auteur, selon le processus désigné par Jacques Derrida par le terme de "destinerrance" 27 , néologisme qualifiant l'évolution du texte, le fait qu'il échappe à son auteur et qu'il mène une existence propre: il est nomade et mobile. Mais on va le voir, de cette existence-là, l'auteur n'est pas exclu ;

27 Jacques Derrida, La Carte postale. De Socrate à Freud et au-delà, Paris, Flammarion, 1999. 
sa participation est même le plus souvent une condition de sa réussite.

On s'intéressera ici aux multiples façons qui permettent à un livre de se faire connaître et aux auteurs de s'exprimer, de dialoguer, de se faire accepter ou reconnaître, bref aux formes de légitimité de l'auteur dans un monde largement influencé par l'Internet. Ces manières de faire émerger l'auteur et son livre s'opèrent selon des modalités différentes. En effet, la prise de parole se fait :

- outil de promotion, avec éventuellement le support des médias et ses vecteurs traditionnels (salons, signatures, conférences, etc.) ;

- médium pour communiquer avec les lecteurs, potentiels, acquis, à conquérir ;

- prolongement ou prélude à l'œuvre papier ;

- vecteur de fabrication de l'œuvre dans le cas des œuvres collaboratives et/ou évolutives.

Les modes de prises de parole et les attitudes des auteurs sur ces questions sont là aussi très diverses.

\subsection{La diffusion hors Internet : les canaux traditionnels}

Globalement, les canaux classiques de diffusion que sont d'une part les interviews dans les médias et d'autre part les salons, évènements en librairie, lectures publiques restent, avant Internet, pour la plupart des auteurs les principaux moyens d'accéder à un public. Presque tous déclarent se livrer à ces différentes formes de promotion, avec toutefois plus ou moins de plaisir.

Les médias classiques demeurent associés à une certaine forme de reconnaissance (comme le fait d'être publié par un éditeur) et donc à un certain prestige. Ils assurent une large couverture et offrent donc une meilleure assurance d'être vu/lu, par rapport à une présence sur Internet. 
Il y a eu un gros succès pour le tome I, c'est passé à Arte, LCl, France 2, il y a eu une page dans Libé, à la radio nationale. [Médecin, romancier, Paris]

Parce qu'un site, si personne n'y va, ça ne sert à rien alors qu'un article, les gens achètent des journaux donc ils peuvent voir l'article et donc aller vers le livre. [...] La page d'un auteur pas connu, personne ne va y aller. [Premier roman, Paris]

L'importance des médias est d'ailleurs très différemment vécue selon le type d'ouvrages publiés. Important pour les romanciers ou les essayistes que nous avons rencontrés, le recours aux médias l'est moins, sans être négligeable, par exemple pour certains auteurs de littérature enfantine. Mais les médias, mêmes spécialisés ou locaux, ne sont accessibles ni à tous les auteurs, ni à tous les ouvrages. Pourtant une certaine notoriété, même limitée, est la condition principale pour émerger en librairie et sur un marché du livre qui édite plus de soixante mille titres chaque année.

Il y a un obstacle [à une meilleure diffusion de mon livre], c'est que je ne suis pas assez connu. Je ne suis pas mis sur table 28 . Je ne suis pas en hypermarché. Mais ça ce n'est pas la faute de l'éditeur, des diffuseurs ou des libraires... [Romancier, Paris]

Participer à des salons, faire des lectures, des signatures ou utiliser d'autres méthodes afin de rencontrer des lecteurs semblent, pour beaucoup, être les moyens les plus directs et les plus accessibles pour "être sur la table " Cette expression, que l'on retrouve de manière répétée chez certains auteurs, signifie bien qu'il s'agit, une fois le long parcours vers la publication accomplie, de continuer le chemin et de sortir de la masse des étagères des librairies et lieux de vente - sans compter que nombre des auteurs n'arrivent même pas jusqu'aux dites étagères. Les voies qui permettent d'accomplir ce saut symbolique, mais aussi très concret, des étagères à la table, ne sont d'ailleurs pas totalement claires pour les auteurs: la vente des livres, l'organisation du marché du livre ne sont pas les seuls critères pour sortir de l'anonymat les auteurs, même s'ils restent déterminants. Jouent, pour les auteurs, d'autres

28 C'est nous qui soulignons. 
facteurs comme les articles de presse, les interventions dans les médias, la fréquentation des sites, l'appartenance locale ou régionale, sans compter les coups de cœur et affinités non seulement des libraires mais aussi des lecteurs. L'Internet semble influer sur ces dynamiques car il confère à l'auteur une initiative dans la conquête et la recherche de son lectorat: l'auteur en effet peut librement faire coexister un blog et la sortie de son livre, alimenter les commentaires des forums sur sa publication (notamment dans le cas d'ouvrages pratiques) ou susciter une polémique ou du buzz via les réseaux sociaux à la sortie de son ouvrage. Ainsi s'amorce un mouvement paradoxal: si le livre numérique peut tendre à déposséder l'auteur de son texte via l'interactivité des formats, l'Internet peut contribuer, dans le même temps, à le rendre davantage acteur de la promotion de son livre en lui donnant des outils idoines d'intervention sur le web. Ses nouveaux moyens participent d'une certaine désintermédiation de la chaîne de valeur du livre, où l'Internet offre la possibilité à l'auteur d'évincer l'éditeur, notamment dans sa fonction commerciale, en accédant par exemple directement à ses lecteurs.

\section{Les rencontres avec le public}

Les rencontres directes avec le public sont des moyens pour les auteurs de parvenir à ces lecteurs si recherchés. S'agit-il pour eux d'une occasion bénéfique, apte à les sortir de leur solitude, évoquée par plusieurs d'entre eux, ou, au contraire, d'une contrainte pénible, difficile à refuser à son éditeur lorsque l'on n'est pas en position de force? Là encore les réponses des auteurs sont assez divergentes. Certains valorisent ces moments d'échanges avec le lecteur et apprécient ces occasions, ou du moins les jugent importantes et fécondes.

J'ai fait un peu de radio (France Culture, comme tout le monde...). J'ai fait des petits bouquins qui me donnaient l'occasion d'être invité ici ou là. Pour mon livre, j'ai fait France inter. J'ai fait aussi Radio Courtoisie... Le travail de soutien, de défense d'un auteur est important; ceux qui ne le font pas se tirent une balle dans le pied. II y a de petits réseaux auprès desquels on peut faire quelque chose. [Philosophe, Paris] 
Mais beaucoup se disent pourtant peu à l'aise dans cet exercice de promotion chronophage, qui peut être fastidieux et ne leur renvoie pas toujours un miroir valorisant. L'un évoque par exemple ces longues heures passées derrière une table, à " attendre le chaland ", sans savoir trop quoi lui dire, alors que le temps de l'auteur, surtout quand il exerce un métier par ailleurs, est si rare. Certains, les mêmes parfois, apprécient d'être extraits de la solitude du créateur de fond.

Les gens viennent en ayant lu ton livre, et ils viennent t'en parler. [...] On va ne pas être d'accord parfois : tant mieux! Le livre leur a parlé, c'est la preuve qu'il ne m'appartient plus. Le livre sort de ma maison, et il me présente des gens, tout comme je leur avais présenté le livre au début. Le phénomène s'inverse, et ça, c'est super, parce que rencontrer quelqu'un d'autre, c'est encore le plus beau voyage qu'on puisse faire. Plus que d'écrire des livres, d'ailleurs. C'est pour ça qu'il ne faut pas trop rester tout seul chez soi non plus, il faut sortir quelquefois, rencontrer le monde. [Jeune romancier, Paris]

Oui bien sûr je fais des salons, des séances de signatures, énormément au début avec mon premier roman mais maintenant, j'ai arrêté, je n'en pouvais plus. Ça vous bouffe un weekend, c'est long et ennuyeux. J'ai une vie très active dans ma profession, donc à côté ça fait beaucoup. Le seul intérêt des signatures, c'est l'autre auteur qui est à côté de vous, pouvoir rencontrer d'autres auteurs. Parce qu'il n'y a pas trop d'échanges avec le lecteur, c'est super rapide. En signature, finalement, on se rend compte qu'on n'écrit pas trop pour les lecteurs : le lecteur passe à côté de ce qu'on écrit. Finalement, la seule personne qui profite de son art, c'est l'auteur. Je le vois bien quand je lis moi-même, on lit en diagonale, le lecteur ne se rend pas compte de tout ce que l'auteur a mis dans le livre. [Médecin, romancier, Paris]

La promotion s'avère parfois une pratique difficile pour des écrivains, notamment les romanciers habitués à s'exprimer par écrit. Plusieurs soulignent ainsi avoir l'impression " d'avoir déjà tout dit " dans leur livre, et éprouver des difficultés à prolonger leur prise de parole lors de ces rencontres avec le public.

Pour le premier, j'ai fait tout : presse, salon littéraire, salon du livre à Paris, foire du livre, lectures en public... et j'ai tout abso- 
lument détesté, parce que, encore une fois, il y a le culte du nom, du visage, au lieu de te parler de ton livre, on te parle de toi : "mais c'est dingue que vous soyez si jeune, vous avez fait quoi avant?". Les gens te regardent avec des grands yeux, et puis en somme tu as l'impression de vendre du poisson. Le saIon du livre de Paris, c'est le grand marché. En plus, tout ce que tu as écrit, eh bien, c'est écrit, justement, tu n'as plus rien à dire! Ça ne sert à rien de parler d'un livre. [Jeune romancier, Paris]

J'en ai fait quelques [lectures publiques], oui, et pour la radio aussi. Mais je préfère quand d'autres me lisent... Ce n'est pas mon métier. [Romancier, Paris]

Les lectures publiques suscitent quant à elles des opinions très mitigées : certains apprécient leur côté "littéraire ", le fait de se concentrer sur le texte lui-même; d'autres rejettent cette situation de confrontation avec le public et son aspect promotionnel qui les met mal à l'aise.

Ouais, alors là c'est moi qui décide des passages, et là ça devient vraiment perso, il y a des passages dont je suis très très fier... C'est là qu'on voit que le bouquin ne m'appartient plus vraiment... On voit les réactions, etc. Je me souviens d'une lecture publique dans la rue pour les 150 ans de mon éditeur, devant les Augustins, on était une dizaine d'auteurs, chacun sur une estrade en train de balancer le texte à la volée dans la rue avec le bus qui passe, le mendiant qui est là... II y avait un côté saltimbanque, médiéval, qui me plaît bien... [Roman historique, Sud-Ouest]

On notera que le réseau des librairies est particulièrement valorisé par les auteurs, que ce soit à travers les évènements que ceux-ci organisent ou la mise en avant de leur ouvrage (coup de cœur de la FNAC, les choix du libraire...) etc.). Les auteurs apprécient à la fois d'être reconnus par des professionnels du livre et de bénéficier d'une promotion en librairie (notamment les plus grandes) qui semble efficace en termes de retombées médias et d'impact sur les ventes.

Les libraires, maintenant, contrairement au début, c'est eux qui appellent [mon éditeur] pour me demander de venir. Ça c'est chouette, parce que la reconnaissance du libraire c'est quelque chose de très chouette; le libraire, lui, sélectionne en fonction de son job. S'il me demande de venir, c'est qu'il sait que ça 
plaît aux gens, donc quand le libraire vient vers moi, ça veut dire que là, il sait que ça va plaire. [Roman historique, SudOuest]

Enfin, les réseaux locaux semblent jouer un rôle réel dans la notoriété d'un auteur: la presse, les libraires et les salons font souvent le choix de mettre à l'honneur un auteur de leur région. De sorte que plusieurs auteurs, parmi les débutants ou peu connus, soulignent avoir bénéficié d'une exposition via ces canaux, qui constituent ainsi une alternative aux grands médias nationaux, plus difficiles à conquérir.

La seule presse qui a soutenu mon truc c'est ma presse natale de Picardie. Les libraires picards ont vachement appuyé. Le Courrier Picard a fait 2-3 articles, j'ai eu plusieurs signatures dans la région. Ça m'a fait plaisir parce que sur la quatrième de couverture j'ai tenu à ce qu'on mette 'originaire de Picardie', parce que j'aime beaucoup ma région et elle me l'a rendu. Après c'est le bouche à oreille. [Romancier, Picardie]

En Corse, on m'appelle beaucoup. Pour les festivals... Maintenant, je suis un peu connue ici. On m'a appelé pour Bordeaux, mais le salon s'est cassé la figure. Sinon, pas plus que ça. [Littérature enfantine, Ajaccio]

Soit je connais les libraires de ma ville, soit c'est l'éditeur qui est en contact avec les libraires et qui va leur demander... [BD, Bretagne]

À côté de ces formes traditionnelles de rencontre entre auteurs et lecteurs, sous la forme de face à face plus ou moins bien organisé, plus ou moins apprécié, se développent des événements où l'intervention de l'auteur est davantage préparée. II n'est pas là (que) pour vendre son livre, mais pour le faire vivre autrement, en discuter, le prolonger. C'est tout particulièrement le cas de la littérature enfantine, des ouvrages illustrés dont les auteurs travaillent par exemple avec des musées, des médiathèques, ou encore des espaces pédagogiques.

II y a 10 jours j'étais à la Tate Modern, dans le hall et j'ai fait dessiner 150 personnes en même temps. (...) Je fais des grands évènements comme ça, je fais dessiner des familles... Tout ça ce n'est pas de la promotion pour moi, c'est un développement. Par hasard à la rentrée je vais faire un évènement, on me prête une salle, moi ça me plaît vraiment, j'aime bien ce 
rapport des grosses audiences avec mes petits livres, c'est excitant. (...) Je fais concevoir, penser, réfléchir. C'est des trucs qui dégagent des envies de travailler. Là j'ai passé la journée à Roubaix dans la médiathèque, 50 personnes de la médiathèque avaient imaginé des ateliers autour de mes livres. [Littérature enfantine, Paris]

Qu'ils apprécient ces occasions ou s'y soumettent nolens volens, les auteurs sont donc conduits à accepter d'être les représentants "physiques" de leur texte, d'en rendre compte aux yeux d'un public. Cela amène deux remarques en conclusion.

D'une part, cette exigence de transmission directe n'est pas réservée aux seuls littérateurs dont la personne serait investie d'une aura particulière; elle touche aussi bien les auteurs de livres pratiques, les bédéistes, les essayistes, etc. : beaucoup sont conduits à parler de leurs livres, à les incarner, à les faire vivre. C'est bien la présence directe de l'auteur qui est jugée par les organisateurs de ces rencontres comme apte à valoriser le livre, à le rendre présent, à le faire exister. Et cette exigence de personnalisation doit certainement être déclinée d'une manière spécifique sur le web ; elle l'est d'ailleurs déjà lorsque les éditeurs poussent leurs auteurs à ouvrir une page personnelle ou le font pour eux; mais cela reste, souvent nous semble-t-il, la version minimale de l'expression individuelle.

D'autre part, la valeur de ces diverses occasions de rencontre en terme de retombées et de diffusion est jugée inégale selon les auteurs (et probablement selon leurs attentes et leurs tirages) mais elles semblent néanmoins des moyens non négligeables sinon de convaincre de nouveaux lecteurs du moins de se faire connaître des intermédiaires du livre, libraires, bibliothécaires, enseignants, amateurs, animateurs culturels, etc. Les intermédiaires là-aussi jouent un rôle central puisqu'ils permettent d'organiser le face-àface et qu'ils sont d'ailleurs souvent les premiers destinataires de telles rencontres. 


\subsection{La diffusion en ligne : outils et modalités}

Internet offre aux auteurs une possibilité de mettre en ligne à peu de frais des textes, interventions, animations, critiques de leurs ouvrages, commentaires, échanges avec les lecteurs, avec d'autres auteurs... et autres multiples possibilités ouvertes par le web. Les producteurs de livre ont des attitudes très contrastées par rapport à cette possibilité, certains en faisant un prolongement de leur activité, voire une activité spécifique d'auteur, d'autres étant plus que réticents.

II semble que la manière d'appréhender et utiliser Internet varie beaucoup en fonction de ces différents facteurs: les compétences numériques de l'auteur, sa plus ou moins grande maîtrise de la parole / communication sur Internet et notamment des réseaux sociaux, fréquemment cités; le type d'ouvrage qu'il écrit (roman, BD, livre pratique, œuvres expérimentales collaboratives); et dans une moindre mesure, son sentiment de légitimité ou non dans la prise de parole. Cette légitimité s'établit notamment par rapport à sa perception du rôle de l'auteur ou par son statut d'écrivain, son degré de célébrité, de présence dans l'espace public.

\section{De l'obligation d'y être et de son coût}

La prise de parole de l'auteur en ligne ne s'avère clairement pas une évidence pour tous, et notamment pour les romanciers, ceux que nous avons rencontrés se montrant plus réticents à s'exprimer sur le web. Plusieurs registres d'explication ont été avancés par nos auteurs. Pour certains, ce type d'ouvrage trouve moins facilement ou de façon moins évidente, un prolongement en ligne par rapport aux ouvrages pratiques et à la BD. Le roman leur semble, plus que les autres types d'ouvrages, correspondre à une œuvre finie, dont la lecture est plus adaptée au format papier et les extensions numériques moins naturelles. Tandis qu'il leur semble plus facile pour l'ouvrage pratique de se décliner en site Internet prodiguant des conseils ou ajoutant de nouvelles thématiques à ce qui a déjà été publié. Certains romanciers conçoivent le travail de l'auteur comme une 
activité laborieuse et solitaire, qui réclame une certaine distance vis-à-vis du public. Á cela s'ajoute la crainte de ne pas " trouver son public " en ligne, d'être peu suivi.

En même temps je ne suis pas sûre que ça intéresserait tant de monde que ça mon blog. Mes livres se vendent très très bien en Poche, mais je n'ai aucune célébrité, je ne suis jamais passé à la télé, personne ne sait qui je suis [...] alors je ne crois pas non plus qu'il y ait une demande qui justifie ma présence. [Romancière, Paris]

Cependant, la multiplication des sites et de blogs d'auteurs littéraires atteste bien que certains auteurs de ce genre peuvent y trouver une autre manière d'être à l'écriture et de s'adresser à un public, avec des formats différents, comme prolongement de leurs œuvres, textes courts ou noms calibrés, échanges avec d'autres auteurs... Dans le cas de la $\mathrm{BD}$, c'est aussi la mécanique commerciale du secteur qui incite à proposer des "échantillons" de BD, quelques planches, pour mieux vendre ensuite l'album à l'éditeur, tel notre auteur du Sud qui a mis en ligne plus de deux cents pages de BD. Certains auteurs d'ouvrages pratiques et de BD utilisent Internet comme outil de promotion ou comme un complément qui valorise leur livre papier. Le lien entre le papier et le numérique paraît alors plus évident, Internet devient une sorte de "mise en bouche " ou un prolongement, un complément de l'œuvre, tel ce vulgarisateur parisien qui souhaite faire un site de culture générale pour que les lecteurs y retrouvent des compléments de ses ouvrages.

Tous les moyens "d'être au public " sont démultipliés sur Internet ; outre les difficultés évoquées, s'y ajoutent pour les auteurs les questions de maîtrise incertaine de leur présence sur le web. Le niveau d'implication par rapport à la présence sur la Toile est très variable, selon leurs compétences techniques en la matière et leur volonté / possibilité d'y consacrer ou non du temps. Leur participation va de l'absence de prise de parole en ligne à la forte implication de l'auteur qui réalise sa propre communication sur Internet, comme en témoignent ces deux positions extrêmes et opposées : 
J'ai fait un site propre à mon livre. [Le web] c'est mon métier de base donc c'est quelque chose qui a été naturel pour moi. Et puis j'ai fait une page Facebook qui a 3000 fans. Je me suis amusé, j'ai fait un petit tirage au sort pour faire gagner deux livres. (...) Les gens venaient, je me suis filmé et j'ai fait le tirage au sort en direct, j'en ai fait une vidéo, postée sur Facebook. Les gens me donnent leur avis, j'ai reçu beaucoup de mails aussi, parce que j'ai fait le Salon du Livre et d'autres salons. [Romancier, Picardie]

Donc, ils [mes éditeurs] m'ont fait une page Facebook, qui ne bouge pas beaucoup, je leur donne parfois des informations qu'ils mettent en ligne. Je ne m'en occupe pas directement. Sur mon site, ça fonctionne de la même façon. Je transmets les infos à propos de mes textes et elles sont mises en lignes. Le site a l'air assez fixe, mais c'est normal, je mets deux ans à écrire un roman, alors le site ne peut pas être très vivant. [Romancier, Paris]

Pour la majorité des auteurs, y compris ceux qui le maîtrisent le mieux, Internet s'affirme donc comme un outil de communication difficilement évitable, permettant de faire connaître leurs œuvres auprès de leur lectorat, mais aussi d'élargir leur public, ou, a minima, de satisfaire aux attentes de leur éditeur. Le web sert de "chambre d'écho" de la promotion plus classique. Une présence en ligne permet aux auteurs de mieux valoriser leur présence physique (off line) à travers leurs ouvrages papier ou leur participation à des évènements (salons, etc.).

La présence sur le web semble donc être devenue une quasi-obligation pour la plupart des auteurs dès lors qu'ils sont entrés dans le processus d'édition. Le fait d'avoir une page à soi peut procéder d'une incitation forte des éditeurs (qu'ils assument ou pas la charge de la page). Elle peut aussi découler d'un choix de l'auteur qui y voit, sans nécessairement trop y croire, un moyen d'amener le public à ses livres ou du moins de tenter de le faire à faible coût (temporel surtout) :

J'ai tout mis en ligne, tous les textes que j'ai écrits, en dehors de contrats de recherche particuliers, sont mis en ligne, sur mon site. Ou sur des sites. J'ai écrit dix, quinze trucs, en plus de mes séances de séminaire que j'ai toutes mises en ligne sous forme de texte ou sous forme audio. Je n'ai aucune con- 
naissance de mes lecteurs. Mon espoir c'est que, prenant connaissance de ce que je fais en ligne, ils vont voir ce que je fais dans mes livres. Mais je ne suis pas du tout sûr que ça se passe comme ça. [Philosophe, Paris]

Demeure pourtant central le problème de l'animation d'un site qu'il convient d'actualiser et de faire vivre, tous les auteurs ne le souhaitent pas, ne savent pas le faire ou n'en ont simplement pas le temps. C'est d'ailleurs toute l'ambiguïté du jeu d'écriture évoqué dans le premier chapitre: la question du style et de la facilité d'écriture dépend aussi du format. Ainsi la rédaction d'un livre ne signifie pas forcément une aisance dans la rédaction d'un blog ou de billets réguliers sur Internet. De la même façon, un auteur attentif à la question de la langue, de l'expression peut craindre de ne pas être à la hauteur de son public qui reconnaîtrait en lui un écrivain, mais risquerait de lui ôter ce statut si ses publications sur Internet s'avéraient décevantes ou simplement triviales (ou même de l'image que lui-même se fait de son style). Le statut d'écrivain confronté à l'Internet est ainsi mis à l'épreuve.

L'investissement de l'auteur peut être beaucoup plus important et le site joue plus qu'un rôle de seule vitrine, presqu'un canal de diffusion complémentaire à l'édition papier, voire, dans certains cas, une nouvelle version à part entière de l'œuvre. L'objectif affiché des auteurs reste néanmoins de mieux servir la diffusion de leur œuvre papier. En effet, selon deux de nos interviewés, une production en ligne de l'auteur non édité semble, par exemple, être pratiquement devenue un critère pour être publié par des éditeurs dans le secteur de la BD, donnant à ces derniers l'assurance que l'auteur possède déjà une audience constituée avant même de publier l'album papier. Dans ces conditions, l'éditeur achète en même temps une œuvre et son public et diminue le risque associé à la publication d'un album. Certains sites sont devenus ainsi des références dans le domaine de la BD.

On part de la BD et on fait un blog commun avec des auteurs qui ont déjà publié en papier, des auteurs un peu connus, avec un partenariat avec les éditeurs pour pouvoir mettre en ligne gratuitement des pages de BD. C'est une plateforme qui attire l'attention. II y a aussi des auteurs [...] qui n'ont pas d'éditeur, 
eux, mettent des pages en ligne quand ils ont le temps. Dans ces cas-là, le risque c'est l'irrégularité des mises en ligne et la prise de risque-en temps et en argent - pour l'auteur si la BD ne plaît pas et qu'il ne trouve pas d'éditeur au final. Pour les auteurs connus, on a voulu travailler en partenariat avec nos éditeurs, ne serait-ce que pour être payé pour ce travail. Le deal c'est une édition normale et en plus l'autorisation de mettre en ligne gratuitement sur [la plateforme]. [BD, Paris]

Pour le web je me sers aussi de notre site, il a un très bon écho: en France il est très consulté pour la BD et pour les comics. Je crée mes propres contenus viraux. Mais tout ça, ça reste du bricolage. [BD, Sud]

On en revient toujours au lourd investissement que représente l'animation continue d'une présence sur le web.

\section{Des divers dispositifs mobilisés}

Cette fonction d'outil de communication s'effectue selon des modalités différentes, selon le type de vecteur Internet utilisé. La plupart des auteurs possèdent, a minima, on l'a dit, une page de type "portrait d'auteur " qui leur est consacrée sur le site de l'éditeur. II s'agit en général d'une page statique, qui comprend une biographie de l'auteur et une brève présentation de ses différents ouvrages (et non d'un livre en particulier). C'est avant tout une vitrine pour l'auteur et l'éditeur. Elle ne permet souvent aucun échange avec les lecteurs ou propose simplement une fonction " contact " (qui n'arrive que rarement dans la boîte email de l'auteur luimême).

Le type d'outils en ligne utilisés varie beaucoup en fonction du genre de livre publié et du niveau de compétences de l'auteur en matière de technologies d'information. Certains auteurs, par exemple, font appel à des personnes de leur entourage, voire des professionnels pour pallier leur manque de connaissances web et de temps, pour se créer une page en ligne.

J'avais créé ce site justement pour faire connaitre mes bouquins, pour faire un blog et créer un lien avec les patients etc. Là il faut le remanier, je n'ai pas trop le temps, mais à la rentrée, je compte remanier mon site et engager quelqu'un pour 
faire vivre le site, parce qu'il faut le ré-alimenter régulièrement. [Nutrition, Paris]

Certains mobilisent tous les outils permettant d'établir des liens, réseaux sociaux, twitts, blogs...

[Ma présence sur le web] est multiple. Je suis sur Facebook, où ce que je mets est très maîtrisé car je connais la puissance de ces réseaux. J'aime beaucoup Twitter. Le blog m'amuse moins, car se faire lire par ses collègues, sa famille... ça enlève pas mal de liberté. Et puis avant j'avais besoin de m'y exprimer pour entrer en contact avec des gens, pour partager. II n'y a plus la notion de plaisir du début, aller à la rencontre des gens. Aujourd'hui je suis en contact permanent avec les gens! Donc aujourd'hui pour moi le numérique est bien plus une présence, une forme d'occupation de l'espace que de création de contenus comme ça l'était au début. Même si le blog me permet encore de poser quelques pensées. [Auteur-blogueur, Paris]

Quelques auteurs de notre échantillon possèdent un site blog dédié à leurs ouvrages (ou à un livre en particulier), plus ou moins développé : simple vitrine de quelques pages web pour certains; site complet interactif ou proposant de larges extraits voire des ouvrages complets pour d'autres, notamment dans le secteur de la littérature jeunesse et de la $\mathrm{BD}$, où l'on trouve les auteurs les plus chevronnés en matière de nouvelles technologies. II s'agit suivant les cas d'un site financé par l'éditeur (plutôt pour les romans) ou crée et financé par un ou plusieurs auteurs (plutôt en littérature jeunesse, BD). Les auteurs un peu plus portés sur les nouvelles technologies disposent d'une page sur les réseaux sociaux comme Facebook sur laquelle eux-mêmes ou leur entourage mentionnent les dates des salons et des séances de signature auxquels ils participent et, pour certains, entrent en interaction avec leurs lecteurs. Les interviewés qui ont le plus de compétences web déclarent assurer euxmêmes la promotion de leur livre sur Internet dans la mesure où leurs éditeurs s'avèrent selon eux peu compétents ou peu volontaires en la matière.

L'éditeur ne fait rien sur ces outils-là, il est complètement largué. Je fais la promo sur le web. Avec l'éditeur, la promo numérique n'est même pas un sujet. (...) Pour le dernier livre d'ailleurs, chez l'éditeur, on m'a fait complètement confiance la 
dessus, pour faire ma promo numérique, en se disant "avec ses 5000 Followers et ses 2000 fans Facebook, ça va se vendre tout seul". C'est faux car l'auteur est la pire personne pour vendre son livre, votre fils est forcément plus beau que le fils du voisin. Par rapport au lecteur potentiel, je n'y crois pas mais les gens qui me connaissent déjà et qui veulent me trouver... [Auteur blogueur, Paris]

La promotion n'est d'ailleurs pas toujours directe, mais peut aussi consister à connaître les bons relais d'opinion, aptes à faire parler du livre, les liens faibles étant toujours, comme l'a montré Mark Granovetter (2000), plus puissants que les relations directes:

J'ai envoyé [mon livre] à deux-trois blogueurs, pas des gens que je connaissais. Je les ai contactés par email et après je l'ai envoyé à deux-trois gros blogs. [Romancier, Picardie]

À cet égard, le blog (décliné en différentes versions: blog seul ou associé à un site, un wiki etc.) s'avère être un outil de promotion et communication particulièrement riche et multi-facette : à la fois vitrine pour l'auteur et ses écrits, et fenêtre privilégiée pour un dialogue personnel avec les lecteurs de par son côté quotidien et pour certains interactif. Le dispositif blog permet ainsi à l'auteur de tester ses écrits auprès des internautes avant publication, mais aussi d'initier des sujets de réflexion ou de faire évoluer des œuvres existantes, en faisant participer d'autres acteurs, tels des lecteurs ou des experts.

Devant la multiplicité des lieux d'expression possibles sur le web, le site ou le blog devient une sorte de compromis entre les différentes modalités de prise de parole, en les associant toutes. II impliquerait un design de la visibilité passant par un équilibre entre le texte et l'image, le support et sa dynamique: ainsi le blog propulserait l'auteur dans un nécessaire travail en amont et en aval de sa publication, faisant du blog lui-même une instance intermédiaire entre l'objet livre et la vie propre du texte.

Je suis très actif sur le web et je pense que c'est essentiel pour mes activités personnelles de chercheur, d'auteur et de journaliste. Je considère mon portail comme mon site phare sur Internet, une vitrine et un lieu de discussion pour presque toutes mes activités sur le web et en dehors. Les deux livres 
que j'ai écrits ont des sites web et [l'un d'eux] est à la fois un blog et une ressource sous forme de wiki. [Sciences humaines, États-Unis]

Dans notre échantillon, peu d'auteurs disposent d'un blog: cela demande une implication personnelle soutenue et requiert une véritable "culture web", avec une certaine notoriété. Comme l'ont montré les travaux de Dominique Cardon et Hélène Delaunay-Téterel (2006) sur les blogs, ces supports du web supposent, pour susciter de l'intérêt, provoquer des commentaires, attirer des lecteurs, des citations, des liens, un fort investissement de leurs auteurs, non seulement dans leur blog mais aussi sur les autres sites d'amateurs, participant aux mêmes réseaux, espaces qu'ils doivent fréquenter et commenter assidûment s'ils veulent la réciproque et donc une certaine visibilité sur le net.

\section{Internet comme moyen d'échanger avec les lecteurs}

Le caractère plus ou moins interactif de l'outil web utilisé amène à un autre type d'utilisation d'Internet: comme moyen de communication, d'échange avec le public ou avec un public particulier. On peut alors s'interroger sur cette évolution du rôle de l'auteur qui assure, au moins partiellement, lui-même sa promotion et définit sa stratégie de communication sur le web. Avec les canaux traditionnels tels les salons, séances de signature, interviews, lectures publiques etc., l'auteur prenait certes déjà la parole, se mettait en scène, mais c'était souvent l'aboutissement d'un travail de relations presse en amont par la maison d'édition. Le contact avec le lecteur semble alors relativement encadré et codifié. Dans le cas du web, la possibilité et les occasions pour l'auteur de prendre l'initiative de communiquer auprès du public sont renforcées, à condition pour lui de maîtriser ces outils et ces modes de communication particuliers. Cela conduit l'auteur à se doter de nouvelles compétences, autres que purement littéraires ou cognitives, artistiques.

Cette activité de promotion est totalement étrangère à certains, notamment les romanciers qui ont plus souvent une vision traditionnelle du travail de l'auteur, soit par manque 
de compétences en la matière, soit parce qu'elle sort complètement du rôle qu'ils estiment être celui de l'auteur.

Je n'ai pas de blog et n'en aurai jamais. Je ne veux pas en avoir. D'abord, parce que je n'aurais pas le temps d'écrire. Ensuite, parce que j'en ai lu certains et que j'ai trouvé ça véritablement inintéressant. [...] Je me cantonne à un truc, c'est un roman qui fait 200, 400 ou 100 pages, c'est déjà suffisamment difficile à écrire pour ensuite aller faire un blog. Certaines personnes sont peut-être très fortes pour le faire, moi je ne sais pas. [Littérateur, Paris]

J'ai une petite page Facebook, mais si je commence avec ça, je n'écris plus, c'est un autre métier. II faudrait un agent pour Internet. [Médecin, roman policier, Paris]

Cette possibilité d'échanger avec les lecteurs est en revanche largement valorisée, par exemple par certains auteurs d'ouvrages pratiques, de BD et quelques romanciers désireux de se faire connaître par l'intermédiaire du web, ou formés aux technologies numériques, familiers de ses outils et désirant en explorer les capacités.

Vous pouvez mettre aussi les commentaires de gens qui interviennent, il y a un échange que vous n'avez pas sur un livre, donc c'est bien. C'est important quand les gens ont quelque chose à apporter, c'est vraiment un apport supplémentaire, on ne peut pas tout connaître, tout savoir, avoir tout compris, donc c'est intéressant des gens qui apportent des choses nouvelles, des références, des points de vue nouveaux, ce sont des continents à découvrir, ça enrichit. (...) Sur le Net, c'est cet échange qui est intéressant, avec des gens qui peut-être n'oseraient pas discuter avec vous quand ils vous rencontrent, et ça je trouve ça très bien. C'est le côté sans frontières du Net qui est très appréciable, ce biais-là est très bien. [Vulgarisation, Paris]

Ainsi, Internet permet principalement aux auteurs les plus " experts " des nouvelles technologies d'être mieux informés, davantage en prise avec leur lectorat et plus autonomes visà-vis de leur éditeur. Le web s'apparente alors aux formes plus traditionnelles d'interactions avec le public, mais sous des modalités différentes, la liberté due à la distance, liberté de s'exprimer, liberté de répondre ou d'ignorer, liberté de ton, liberté liée à l'a-synchronie des échanges, voire à leur (relatif) anonymat. On notera que la taille du public touché 
est bien sûr très variable : le public potentiel peut être plus large si le site arrive à susciter un certain trafic et que les possibilités et les modalités d'expression sont variées et multiples, notamment à travers les réseaux sociaux tels Facebook, l'utilisation de Twitter, la participation à des sites et les blogs d'auteurs.

Pour ceux qui maîtrisent le mieux le web, Internet devient l'occasion d'un rapport direct avec le lecteur qui ne paraît pas tout à fait anodin, et dont le blog pourrait être vu comme l'instrument privilégié. Alors qu'il existe classiquement une certaine distance de l'auteur par rapport au lecteur, sur Internet, le lecteur se trouve en position de s'adresser directement à l'auteur, y compris pour lui demander d'expliquer son œuvre. On peut ainsi se demander dans quelle mesure ce nouveau rapport fait évoluer les notions d'auteur et d'œuvre: Internet contribue-t-il à rendre plus prégnante, mais aussi plus proche du lecteur, la personnalité de l'auteur? Dans quelle mesure Internet établit-il un lien plus étroit entre l'auteur et son œuvre, cette dernière étant considérée auparavant en elle-même et indépendamment de son interprétation par l'auteur?

[A propos du jeu organisé sur son blog] Mon éditeur se demande si ça ne risque pas de désacraliser l'auteur. C'est une vraie question de fond: est-ce que l'auteur doit être aussi proche que ça du lecteur, doit-il garder une distance ou pas? Je n'ai pas la réponse. Moi, j'ai beaucoup aimé, pour moi c'est le même contact qu'on peut avoir au Salon du Livre par exemple. Après c'est beaucoup d'énergie. II faut animer la page, si on l'ouvre il faut être capable de la suivre, de répondre, sinon ça reste narcissique, péremptoire. II faut être capable de créer les conditions de dialogue, ça prend du temps. [Romancier, Picardie]

On remarquera que se dessine une nouvelle économie de la subjectivité de l'auteur, c'est-à-dire de nouvelles formes d'identités, fondée sur son accessibilité numérique, qui se construit par la diffusion d'informations ciblées, l'objectif étant de créer une cohérence avec la publication du livre et de donner une explication à la logique de publication. Ainsi, l'auteur peut influer sur le choix du lecteur, l'orienter vers ses ouvrages. La question du référencement par les moteurs 
de recherche devient centrale dans la visibilité de l'auteur sur la Toile.

À cela s'ajoute la réciproque: l'influence du lecteur sur l'auteur. On l'a vu, certains auteurs de l'échantillon qui animent eux-mêmes leur blog s'en servent comme une sorte de " soupape de sécurité ", pour bénéficier d'un retour rapide et vérifier l'intérêt de leurs écrits auprès des lecteurs à une petite échelle. On parle des "comités de lecture " constitués par des maisons d'édition, parfois composés de lecteurs non professionnels, afin de s'assurer de l'intérêt d'un manuscrit pour le lecteur, comme cela est aussi fait pour l'écriture de séries télévisées. On semble là s'inscrire dans une logique similaire, un processus où le lecteur peut intervenir pour orienter la conception de l'œuvre.

Je ne permets pas l'affichage des commentaires sur mes posts. Je les reçois, je les lis et bien souvent j'en tiens compte dans mon travail quand ils sont pertinents. C'est souvent le cas d'ailleurs avec les jeunes lecteurs de ma série XX, ils posent des questions, relèvent des incohérences... Ça me permet de revoir les intrigues, de faire évoluer les personnages ou leur présence... [BD, Paris]

Cette influence du lecteur peut d'ailleurs être indirecte, l'auteur attendant moins des commentaires directs sur son œuvre que des opinions, des anecdotes, des débats. Cela rejoint ce que nous avons vu plus haut: Internet comme "baromètre de l'époque ", Internet devenant un moyen pour l'auteur de tester la validité de ses approches ou de ses observations, Internet s'ajoutant aux multiples moyens traditionnels par lesquels les auteurs s'informent.

\section{Conclusion intermédiaire : portraits de l'auteur comme internaute}

Si l'on s'intéresse aux auteurs qui ont une activité numérique au moins minimale, on peut donc les classer selon deux axes:

- en abscisse, on distingue les écrivains qui organisent leur activité d'internautes comme un prolongement de leur activité d'auteurs de livres publiés, versus ceux 
pour qui l'activité d'internaute n'est pas directement articulée avec la publication d'ouvrages. L'axe mesure donc l'articulation entre les écrits électroniques de l'auteur et ses écrits papier.

- en ordonnée, on distingue les auteurs qui utilisent Internet comme un espace d'expression (éventuellement parmi d'autres) versus les auteurs qui en sont davantage les témoins que les acteurs; ces derniers délèguent alors à d'autres (amis, lecteurs, éditeurs...) l'essentiel de l'animation d'un site ou l'assurent a minima. Cet axe mesure donc le degré d'implication directe de l'auteur sur le net.

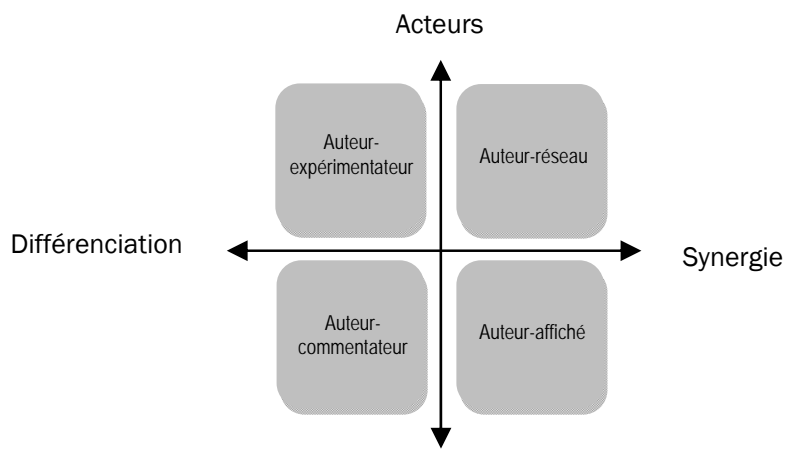

Témoins

Ce qui dessine quatre idéaux-types de l'auteur-internaute qui correspondent à ce que l'on pourrait désigner comme quatre niveaux de la "conscience numérique ", c'est-à-dire l'ensemble des pratiques qui vont de la simple acclimatation à Internet à une appropriation intense.

- L'auteur-expérimentateur est un internaute zélé, activiste de l'écriture en ligne, mais il met de la distance entre ses activités d'auteurs de livres publiés et celles d'auteur du web. C'est le cas de notre jeune écrivain, par exemple, qui anime un site consacré aux nouveaux auteurs, sans mettre sa production au centre de son activité d'internaute et en tenant soigneusement à dis- 
tance ses propres productions littéraires. L'auteurexpérimentateur peut se servir de ses pratiques sur le web pour mener à bien des expériences créatrices, pour poursuivre des activités liées à ses pratiques professionnelles. Mais il ne l'utilisera pas directement pour montrer son travail, le faire vivre. Si son activité d'internaute a un impact sur la diffusion de ses ouvrages, cela sera indirectement (ce qui ne veut pas dire d'ailleurs que ce sera sans efficacité). Internet est d'abord un espace disjoint de l'œuvre papier.

- L'auteur-réseau a une forte "conscience numérique". Typiquement, il s'agit de l'auteur en sciences-humaines de notre échantillon qui envisage ses travaux comme des livres ouverts, évolutifs et collaboratifs, dont les mises à jour peuvent donner lieu à une nouvelle édition. Ici, les internautes participent directement à l'élaboration du livre proposé en ligne par leurs commentaires et éventuellement leurs interventions directes dans le texte. L'auteur envisage donc Internet et le numérique dans ses différentes composantes : un vecteur de continuité de son activité créatrice, intimement lié à sa pratique d'écriture, et au-delà, un outil de production, diffusion, échange, qui fonctionne en synergie. En somme, un "hypermédia " qui fait du numérique, une partie intégrante de l'œuvre, un espace réticulaire de génération de l'œuvre. Dans certains cas, l'œuvre numérique précède l'œuvre papier: l'activité en ligne peut même aboutir à la publication d'un livre papier et la notoriété de son auteur précéder la publication dudit ouvrage.

- L'auteur-commentateur a une conscience numérique émergente ; il anime un site, il commente ses activités, signalant ses rencontres, ses lectures; bien souvent cela lui a été présenté, telle notre auteure corse, comme une nécessité pour la diffusion de ses œuvres dans laquelle elle doit directement s'impliquer ; ses activités en ligne montrent ses compétences d'auteur, tout en ne se limitant pas au " service après-vente " des travaux publiés; il montre sa capacité à élaborer un discours hors des livres. Support à la création, Internet 
est ici vécu comme un espace informationnel élargi qui vient alimenter la réflexion de l'auteur.

- L'auteur-affiché, affiche une conscience numérique faible qui procède parfois d'une posture revendiquée: à savoir le modèle d'un auteur insulaire, dégagé d'un numérique jugé contingent. C'est particulièrement vrai de certains romanciers. C'est souvent un éditeur qui impulse sa présence en ligne et qui vient lui en suggérer la pertinence. En effet cet auteur n'est pas familier des espaces numériques et considère que son rôle d'écrivain ne relève pas d'une activité sur le Net. II ne contribue qu'indirectement ou faiblement aux parutions du site de l'éditeur. Le site ainsi envisagé est vécu comme un simple support de ses activités d'auteur de livres publiés. Internet est ici vécu comme un espace élémentaire de diffusion et de promotion.

Ces idéaux-types, construits à partir de notre échantillon d'auteurs interviewés et d'une exploration des sites d'auteurs, mériteraient d'être confrontés à un panel plus large, mais ils dessinent d'ores et déjà un tableau des "auteurs en Internet" que nous avons souhaité incarner par des portraits d'auteurs, inspirés par quelques-uns de nos entretiens.

e) Brefs portraits d'auteurs dans le monde numérique

\section{L'auteur expérimentateur}

Notre auteur expérimentateur est un graphomane : il écrit partout, tout le temps, sur tous les supports et par tous les temps. De la littérature souvent, des travaux académiques parfois, des mails, posts, twitts de manière continue. Tellement d'ailleurs qu'il désire même transmettre sa passion et faire écrire les autres. II se dit aussi le "frère jumeau " de l'Internet: l'écriture est avant tout pour lui une affaire numérique. II ne jure que par les réseaux sociaux, s'est créé son propre site Internet, est particulièrement bien référencé et adore intervenir dans les forums de discussion littéraires. II possède toutes les compétences requises pour assurer la promotion de ses ouvrages en ligne et n'hésite pas à s'en servir. II présente des extraits de ses ouvrages sur son blog, tient à jour les dates de ses prochaines séances de signature sur sa page Facebook et 
annonce la sortie de son prochain livre à ses Followers sur Twitter. II en sait plus que son éditeur sur la promotion en ligne. Internet représente pour lui une alternative aux médias traditionnels et lui permet de toucher directement les lecteurs pour accroître sa notoriété. Loin de la conception insulaire de l'auteur affiché, il ne craint pas d'entrer en contact avec ses lecteurs, de se montrer à l'écoute de leurs avis en ligne, quitte à désacraliser l'image de l'écrivain. Pour autant, il tient toujours à la publication papier et la promotion sur Internet lui sert à mieux assurer la diffusion de son œuvre dans le monde physique. Si son livre est distribué en version numérique, il s'agira d'un livre homothétique, ne faisant que dupliquer la version papier et non destinée à évoluer avec les commentaires des internautes. Le livre papier reste l'expérience finale, l'aboutissement accompli de son travail d'auteur.

\section{L'auteur commentateur}

Notre auteur commentateur n'est pas a-numérique, on pourrait plutôt dire qu'il est analogique: ses pratiques d'écriture en ligne sont en continuité avec ses activités d'auteur; les livres enrichis, l'écriture collaborative et évolutive, les wikis ou les twitts ne font pas partie de son univers. II se sert d'Internet au quotidien pour communiquer par email, pour chercher des informations, vérifier ses sources, voire trouver des thèmes qui l'inspireront et nourriront sa réflexion. Parfois, il y cherchera un éditeur ou un illustrateur. Internet est avant tout pour lui un nouveau "média " qui lui ouvre une fenêtre de plus sur la société: il flâne sur les forums comme on écoute une conversation au bistrot, lit les blogs ou les sites de presse comme il feuillète les journaux, bref il y hume l'air du temps. Internet l'aide à compléter son travail d'information mais n'intervient guère dans ses pratiques d'écriture. II sait bien pourtant que la notoriété et la visibilité d'un auteur se construisent désormais sur la toile : il a donc accepté la proposition de son éditeur qui l'a incité à ouvrir un blog ; comme son maniement est intuitif, qu'il n'est pas encore assez connu pour en confier les clefs à son éditeur ou à son agent, et qu'il aime bien, finalement, raconter des histoires, faire partager son enthousiasme pour telle lecture, rendre compte de telle séance de signature inattendue ou d'une rencontre plaisante, il se résout à le nourrir, se prenant parfois même au jeu du journal. II sent bien que son investissement est un peu trop limité et qu'il faudrait qu'il y consacre plus de temps pour avoir une 
véritable présence sur la toile, mais là n'est pas sa préoccupation dominante et il refuse que le numérique envahisse par trop son univers d'auteur.

\section{L'auteur réseau}

Internet est pour cet auteur là son univers de prédilection ; il le maîtrise et même mieux, il le laboure, il le cultive, il le transforme. La toile lui a ouvert un nouveau champ de possibilités en permettant une écriture ouverte, collective, évolutive, continue. II explore tout ce qui permet de bâtir de l'écrit numérique, la dimension collaborative, l'hyper textualité, le caractère multimédia, la multiplicité des formes expressives... Les commentaires, modifications et ajouts des internautes participent pour lui entièrement de l'œuvre, écrite à plusieurs mains et sous licence Creative Commons. Son travail bouleverse la conception classique de l'œuvre finie, aux frontières clairement délimitées, pour ouvrir de nouveaux horizons avec une œuvre numérique encore à définir. L'œuvre papier devient un produit parmi d'autres de sa production, un moment d'arrêt dans un travail, mais pas nécessairement le support le plus important ; s'il peut, il récusera toujours l'exclusivité du papier lui préférant la multiplicité des formats. II pense que le réseau lui rendra d'une manière ou d'une autre ce qu'il lui a donné, en notoriété, en relations mais peut-être même aussi en ressources, directes ou indirectes. II pourra privilégier des plates-formes d'autopublication, comme Lulu.com, dans lesquelles l'éditeur n'est plus au centre de la chaîne de valeur. Mais il ne s'interdira pas non plus les éditeurs traditionnels, dès lors toutefois qu'ils acceptent la mise en ligne gratuite d'une version numérique de son travail, et les conditions de diffusion et de communication qu'il détermine.

\section{L'auteur affiché}

L'auteur affiché sépare l'écriture de sa technologie, le livre de sa diffusion ; à chacun ses outils et à chacun son rôle ; le sien est d'écrire dans la solitude et l'épreuve de l'écrivain. II revendiquerait aisément un certain "artisanat " de l'écriture tant il est attaché à ses "outils": le papier, la plume, le cahier, et même l'ordinateur, dès lors qu'il n'est pas connecté, qu'il est un instrument d'écriture personnalisé, qu'il permet le face-àface entre le texte et son auteur. Bien sûr, il a un mail ; comment communiquer sinon rapidement avec son éditeur, mais 
c'est juste un instrument commode, pas un mode d'expression en soi. Bien sûr, son éditeur l'a convaincu qu'il ne pouvait plus faire l'économie d'une présence sur le web, que sa réputation et sa notoriété se construiront aussi là. C'est un ami qui l'a donc débarrassé de ce souci, lui créant une page qu'il fréquente rarement et à laquelle il participe encore plus rarement. Le livre numérique peut l'amuser: voici, selon lui, un support ludique et s'il n'a pas la noblesse du livre traditionnel, il peut aussi ouvrir des pistes créatives, mais sûrement plus pour les autres que pour lui. Le livre enrichi ? II ne connaît pas. En aucun cas d'ailleurs, il n'accepterait des intrusions écrites autres que les siennes dans son texte. Son œuvre singulière passe par l'écrit, tout comme la pensée.

\subsection{Un nouveau rôle pour l'auteur?}

\section{L'émergence d'un nouveau profil d'auteur?}

Environ la moitié des auteurs de notre échantillon exerce régulièrement et par ses propres moyens une prise de parole en ligne. On peut se demander si n'émerge pas un profil d'auteur qui sera à l'avenir mieux formé aux nouvelles technologies et d'une manière générale plus informé et en prise avec la société et son lectorat. En somme, un auteur "connecté " dans tous les sens du terme.

On notera que pour certains auteurs l'expression "auteur connecté " reste antinomique, notamment comme nous l'avons déjà souligné chez ceux des romanciers qui sont très attachés à un modèle d'auteur traditionnel. Pour eux, cela interroge directement le processus de création qui peut se trouver modifié par les impératifs et vœux des uns et des autres. Le livre perdrait ainsi de sa singularité pour s'acheminer vers un livre mashup ${ }^{29}$ où la notion de dépositaire de l'œuvre est peu à peu diluée. Certains de ces auteurs se placent dans la perspective de Pierre Bourdieu et

29 Le mashup est une pratique artistique consistant à agréger des sources et des styles artistiques différents, afin de générer un nouveau contenu. C'est une pratique qui tend à se répandre en musique et en vidéo. 
de sa hiérarchie des arts, en estimant qu'on irait vers une sorte "d'art moyen ", telle selon lui la photographie. La recherche de consensus se ferait, craignent-ils, au détriment de la vision propre de l'auteur. Ces questions auxquelles les auteurs et les éditeurs sont confrontés viennent d'ores et déjà alimenter les débats sur le statut du livre numérique. Questions d'autant plus cruciales qu'elles interrogent l'ensemble de la chaîne du livre, le statut juridique de l'œuvre et le droit de propriété de l'auteur sur son écrit.

Nos auteurs, parmi ceux qui sont les plus gros "pratiquants numériques ", signalent aussi la réticence, voire la méconnaissance du web et de ses capacités de création, de diffusion et de communication de nombreux éditeurs.

[Mon] éditeur est largué. J'ai assuré la promotion sur le web. Quand on a fait le livre pour iPhone, il a pris quelqu'un en indépendant dont c'était le métier et, ça n'a rien eu à voir, on a eu cent articles dont des articles en italien et en anglais. II a vraiment beaucoup travaillé et ça a très bien marché. II a ciblé des lecteurs potentiels, des journalistes adaptés. Cette personne a été très déçue de ne pas se voir confier la promotion du livre papier mais il fallait deux mille euros que je n'avais pas et ça a été un suicide, un vrai échec. C'est un scandale de ne pas avoir bossé plus pour la promotion de ce livre. [Auteur-blogueur, Paris]

Bien peu d'éditeurs semblent, d'après nos auteurs connectés, prompts à mettre en place de cellules de community management sur le Web afin de "buzzer " autour d'un nouvel auteur ou de créer des groupes Facebook relatifs à une maison d'édition ou à une ligne éditoriale. Dans un cas pourtant, celui des livres du docteur Dukan qui ont occupé la première place dans le palmarès de vente pendant de très longs mois l'éditeur (ou son agent) est crédité d'une opération réussie de marketing, appuyée sur l'Internet, alors qu'il était ignoré des relais d'opinion traditionnels.

Oui, on est à l'heure des blogs, ce qui fait marcher les bouquins, ce sont les blogs, comme pour le bouquin de Dukan ${ }^{30}$,

30 Dans l'enquête du ministère de la culture, Économie du livre, de mars 2011 (déjà cité), on note que, en 2010, les ouvrages de Pierre Dukan occupent, en 
qui doit son succès à la blogosphère. C'est un cas de merchandising très bien pensé. Ce lancement a été remarquable, c'est presque un cas d'école. Le bouquin est sorti en 2001 et il n'avait pas de succès au départ. Le succès est venu plus tard, quand les filles blogueuses ont commencé à en parler. C'est le succès de la blogosphère. [Nutrition, Paris]

S'il est difficile de se livrer ici à un exercice de prospective, on peut toutefois souligner les paradoxes qui semblent se mettre en place et devraient peser à l'avenir sur les auteurs. Le rapport à la technologie ne va pas toujours de soi, on l'a dit. L'auteur se réclame parfois d'une position de retrait et parfois revendique la nécessité d'une exposition sur Internet. Le rapport au temps du web, dans la quasi-immédiateté et la vitesse accélérée bouscule ou interroge aussi celui de la publication papier. On observe de grandes différences entre les approches des auteurs, leur exposition sur le web moins selon le ou les genres d'écriture dont ils relèvent qu'entre leur degré de familiarité, de compétence mais surtout d'intérêt pour l'expression numérique.

\section{Internet, un vieux média tout neuf ?}

Certains auteurs qui bénéficient déjà d'une certaine notoriété ont tendance à situer Internet au même niveau que les vecteurs traditionnels de promotion que sont les salons, les séances de signatures ou les interviews et articles dans les médias classiques. II s'agit plutôt pour eux d'un outil promotionnel supplémentaire plutôt que d'une nouvelle façon de faire connaître son livre.

C'est une autre façon de faire voyager le travail, de le faire connaître. Autrefois par exemple, nous recevions beaucoup de courriers, aujourd'hui ce sont les emails. C'est donc une évolution, mais pas une révolution. Toutes ces choses existaient déjà avant le numérique. [Homme de lettres, Paris]

Pour d'autres, notamment parmi les auteurs "débutants", Internet s'affirme en revanche comme une alternative aux

nombre d'exemplaires vendus, trois des quatre premières places (la brochure de Stéphane Hessel complétant ce peloton de tête en arrivant deuxième). 
médias traditionnels, un moyen de les contourner et donc d'émerger parmi la grande quantité d'ouvrages qui paraissent chaque année. Internet a ainsi constitué un précieux canal de communication pour plusieurs auteurs de l'échantillon qui n'auraient pas eu un écho auprès des lecteurs aussi important sans lui. Le web s'avère de plus être un média relativement flexible dans la mesure où il permet de s'adresser à une population/ communauté particulière.

II y a une chose que je trouve un peu triste, c'est que la presse d'une manière générale tourne un peu autour des mêmes auteurs, la rentrée littéraire est un peu caricaturale pour ça. Qu'on prenne tel journal ou tel autre, c'est finalement les mêmes livres dont on va parler, il n'y a pas beaucoup de place pour les nouveaux. [...] Globalement, ce qui a permis à mon livre de vivre, c'est que j'ai été soutenu par des blogueurs. [Romancier, Picardie]

Une des qualités reconnues de l'Internet pour les auteurs semble être sa capacité à se distinguer du petit cercle de ceux qui parlent des livres sur la place publique et les font vivre dans les principaux médias, un cercle qui leur semble trop consanguin, trop fermé. Les blogs ou sites d'amateurs semblent jouir d'une capacité d'indépendance qui laisserait, pour eux, plus de place à des auteurs non établis. C'est d'autant plus vrai lorsque l'auteur ne s'appuie pas seulement sur les espaces consacrés aux livres mais peut aussi obtenir le soutien d'autres amateurs, comme dans le cas de ce romancier avec les joueurs de cartes:

Globalement, ce qui a permis à mon livre de vivre, c'est que j'ai été soutenu par des blogueurs. J'avais essayé de voir comment ça marchait. J'ai envoyé le livre à quelques blogueurs et ils m'ont suivi. Vous tapez sur Google [le titre de mon livre], vous verrez, il y a pas mal de blogs qui remontent. (...) Et puis j'ai eu un autre soutien sur Internet, c'est le site XX.net, qui est un site communautaire de joueurs (...) ; la communauté de joueurs sur Internet m'a vachement aidé. J'avais peur que les joueurs trouvent le roman pas assez technique, parce que ça peut être lu par tout le monde, pas que les joueurs. (...) Je l'ai envoyé à deux trois blogueurs, pas des gens que je connaissais, je les ai contactés par email et après je l'ai envoyé à deux ou trois gros blogs. Notamment le blogueur XX qui est assez connu dans le milieu (...) qui a fait un article sur mon roman, après par rico- 
chet c'est arrivé chez XX qui est le rédacteur de l'émission de Canal+ (...) et donc je suis passé sur Canal+ sur l'émission du jeudi (...) Quelques lecteurs "fans ", sans que j'en parle ont échangé, avec une blogueuse, et m'ont dit qu'elle aimerait parler avec moi, c'était quelqu'un d'un site de lecteurs, elle m'a dit je veux absolument le proposer, et donc le fil s'est tiré comme ça. J'ai eu la chance que la rumeur a été positive, ce n'est pas le buzz du siècle, mais ça a permis au livre d'exister. [Romancier, Picardie]

Ce rôle joué par les lecteurs internautes, dans la promotion et la revue critique des livres, pose la question du statut de l'information et de la critique sur Internet par rapport aux médias traditionnels. Cette réappropriation par des lecteurs plus ou moins avertis (notamment des blogueurs), d'un rôle jusque-là réservé à grande échelle (le bouche à oreille a toujours existé) aux critiques littéraires et libraires, donc à des professionnels, amène à s'interroger sur la valeur de la reconnaissance de l'auteur par les internautes. Peut-on parler d'une nouvelle forme de reconnaissance/justification du statut de l'auteur par l'aval (le lecteur) par opposition à l'amont (l'éditeur, le journaliste, le libraire), et par l'amateur par opposition au professionnel ? L'opposition entre amateurs et professionnels est remise en cause sur Internet, certains sites s'avérant, selon nos auteurs, de très bonnes références dans leur domaine de compétence; comment, dès lors, rendre compte de la qualification de ceux qui écrivent sur le web? On voit émerger un nouveau type de médiateurs des livres, à l'efficacité avérée. On pourra alors se demander si et sous quelles conditions cela transforme la chaîne du livre ; c'est en tous les cas une interrogation des auteurs que nous avons rencontrés.

Dans la reconfiguration des intermédiaires, se pose aussi la question du rôle de l'éditeur: si la plupart des auteurs n'envisagent pas de s'auto-publier et restent très attachés à l'éditeur qui apparaît à la fois comme un signe de reconnaissance du statut d'auteur et comme la clef d'entrée dans la filière du livre, dans certains cas plus marginaux, c'est le lecteur qui a "fait " l'auteur. Cela a été le cas, on l'a dit, pour le blogueur auteur de l'échantillon, dont le succès en ligne à travers son blog, a conduit des éditeurs à s'intéresser à lui. II 
avoue cependant avoir eu des difficultés au départ à se voir comme un "auteur".

J'ai commencé mon blog [...] il y a 7 ans, j'avais 31 ans à l'époque. L'idée était d'exprimer mon expérience, mon vécu du métier, j'étais fatigué. Le blog était pour moi une bonne manière de raconter ce que je vivais, tout en fictionnalisant un peu pour ne pas que les gens puissent se reconnaître, mais globalement tout était vrai et j'avais besoin de la raconter, il n'y avait pas de groupe de parole. Ce blog a rapidement très bien marché et j'ai été démarché par plusieurs éditeurs au bout d'un mois et demi - deux mois. Au début j'ai refusé, je ne voyais pas ce que j'allais faire là-dedans, et puis au troisième ou quatrième, j'ai fini par accepter, j'étais amusé, je me sentais comme un imposteur. Je l'ai fait aussi car beaucoup de gens le demandaient sur le blog. [Auteur blogueur, Paris]

La notion "d'expérience ", de "vécu " est ici mise en avant par l'auteur blogueur comme le fondement de sa démarche. Celle-ci est vécue sur le mode du récit et mêle une part d'autofiction à l'élaboration de son blog et plus tard de son livre. Le blog est en quelque sorte vécu comme un vivier, un support de mémoire qui permettra par la suite de donner naissance au livre. De fin en soi, l'auteur conçoit son blog progressivement comme un moyen d'alimenter un nouveau processus de création : on voit là un intéressant lien entre auteur-blogueur et auteur au sens de livre papier. Le processus créatif évolutif qui associe différents formats fait aussi la part belle aux lecteurs internautes qui permettent de créer un intérêt chez d'autres lecteurs mais aussi chez les éditeurs.

\section{Les œuvres collaboratives et évolutives}

Certains auteurs vont jusqu'au bout de la logique faisant participer le lecteur au processus de création, en mettant en place un processus évolutif et/ ou collaboratif de production de contenus. L'ouvrage est ainsi produit "à plusieurs mains ", sur le modèle de l'encyclopédie en ligne, dans un processus itératif, et qui ne porte pas de date de fin.

Oui j'anime et je coordonne le développement de ce livre. Les auteurs m'envoient leur matière brute, textes et images, et c'est moi qui m'occupe d'harmoniser et mettre en forme. (...) II 
n'y a pas de date limite, le livre collectif se constitue et se constituera à mesure des apports des auteurs. Le livre est donc à la fois collaboratif et collectif, car il ne pourrait exister sans l'activité des experts bénévoles qui écrivent le chapitre, et évolutif car on ne prévoit pas de date limite du projet a priori et l'ouvrage est entre temps disponible à la lecture en ligne, pour tous, pendant ses phases intermédiaires. Je crois d'ailleurs que beaucoup des nouveaux auteurs ont été plus intéressés à collaborer parce qu'ils pouvaient déjà suivre les travaux euxmêmes, plutôt que de se limiter à consulter quelques lignes guide et la foire aux questions. [Auteur de collectif, Paris]

L'œuvre est réévaluée en permanence, n'étant ainsi jamais réellement achevée. L'auteur devient une sorte d'agrégateur de contenus et donc son nom joue davantage comme une marque qui garantit la qualité du résultat final.

Lulu est une entreprise qui comprend les changements de la communication. C'est un service d'auto-publication, une opération qui permet de faire de mon travail un livre qui peut être vendu par moi et par toute autre personne qui souhaite le distribuer. A l'automne dernier, quand j'ai commencé à travailler sérieusement sur le livre lié au projet, j'étais sous contrat avec l'éditeur qui a publié mon livre précédent... il y a quelques années. On a décidé de mettre fin à notre collaboration en janvier et mon agent littéraire a commencé à chercher un nouvel éditeur. Mon ancien éditeur n'avait pas de problème avec les Creative Commons, nous avons d'ailleurs publié le premier livre sous cette licence. Mais comme me l'a dit David, mon agent, lorsqu'on commençait notre recherche d'un nouvel éditeur, les possibilités risquaient d'être limitées parce que j'avais un unique critère non négociable: que le livre soit publié sous licence Creative Commons. [Sciences-humaines, États-Unis]

On peut se demander si, avec ce type d'ouvrage dont l'objectif est clairement d'évoluer en permanence, on est encore face à une œuvre, celle-ci semblant impliquer l'idée d'un produit fini. Se dessinerait davantage un produit "nomade " et non plus " sédentaire ", une œuvre alimentée par une mobilisation continuelle des contributeurs et une posture de qui-vive de l'auteur. Dès lors, la notion de réédition de l'œuvre est particulièrement importante pour l'auteur. Dans le cas des livres pratiques par exemple, la plus-value de la réédition procèdera de la synthèse pertinente des apports des lecteurs, de leurs réflexions. La plasticité du 
format numérique permettra d'autant plus aisément de procéder à des modifications: néanmoins, comment l'auteur voit-il ces ajouts? S'ils peuvent certes apparaître comme des enrichissements, ils peuvent aussi contribuer à dissoudre la notion d'auteur unique, dépositaire de son œuvre et orienter une évolution vers des collectifs d'auteurs.

\subsection{Le piratage, entre notoriété et rémunération}

L'attitude qu'ont les auteurs vis-à-vis du piratage est directement liée à la conception qu'ils se font du droit d'auteur, à ce qu'ils en attendent et à la manière dont ils évaluent la chaîne de valeur du livre.

\section{Genres, notoriété et droit d'auteur}

Le droit d'auteur n'est en fait pas perçu tout à fait de la même manière suivant le type d'ouvrage produit. On distinguera ici, par exemple, les romanciers d'un côté et les auteurs d'ouvrages pratiques et BDistes de l'autre. Mais surtout la carrière des auteurs et la manière de construire leur notoriété diffèrent.

Pour les romanciers, outre l'aspect financier qui demeure important, le droit d'auteur semble bénéficier d'une dimension symbolique. Beaucoup d'entre eux ont ainsi souligné qu'ils n'écrivaient pas pour les revenus générés (d'ailleurs minimes pour le plus grand nombre) mais d'abord pour être lus, pour le statut, la reconnaissance que cela procure. II semble en effet que c'est à partir du moment où l'on est publié et que l'on touche des droits d'auteur, que l'on accède au statut d'auteur, c'est-à-dire de "professionnel de l'écriture ", non comme activité permettant d'en vivre, mais comme différenciation avec ceux qui écrivent pour eux et qui n'accèdent pas à l'autorité de l'espace public ${ }^{31}$; ces derniers

${ }^{31}$ Ainsi que l'exprime fort bien un de nos interviewés : "Depuis que j'ai réussi à écrire un livre de la première à la dernière ligne en le maîtrisant, ça veut dire 
étant fort nombreux mais sans doute de moins en moins à mesure que l'internet ouvre ses possibilités, sinon de lecture, du moins d'écriture publique. On voit ainsi émerger une tension entre l'auteur professionnel et la profusion d'amateurs. Pour des auteurs, souvent acclimatés à la sociologie de Pierre Bourdieu, en effet, le statut social et la légitimité de l'auteur en tant qu'acteur du champ artistique, sont des enjeux symboliques forts. La " conquête " de ce statut est aussi vue comme une volonté de distinction qui permettra à l'auteur d'être lu, entendu et écouté. Le droit d'auteur est l'élément tangible de cette reconnaissance ; l'idéal semblant être, pour certains la possibilité de "vivre de son œuvre", quand d'autres tiennent à leur statut double: écrivain ET médecin, écrivain ET policier, universitaire ET auteur, etc.

Derrière ce statut symbolique de l'auteur se cachent donc les concepts de notoriété et de reconnaissance, concepts complexes à identifier et plus encore à mesurer. Dans son analyse économique de l'exploitation et de la production de la notoriété médiatique, Françoise Benhamou, parlant de L'Economie du star-système (2002), pose la question de la notoriété artistique et/ou médiatique des stars. Pour elle, deux sortes de notoriété se font face. D'un côté, la notoriété artistique qui représente le talent artistique reconnu à l'artiste par la société ; elle peut être estimée, par exemple, grâce aux récompenses obtenues. De l'autre, la notoriété médiatique qui, elle, est définie par le fait d'être connu de manière large et généralisée, presque indépendamment du talent de l'artiste ; cette notoriété est estimée, par exemple, par le nombre d'articles parus dans les médias (presse, télévision, radio, etc.). Dès lors, si certains, comme Rosen (1981) et MacDonald (1988), considèrent la capacité d'attraction de l'artiste comme fonction de son niveau relatif de notoriété artistique, pour d'autres en revanche, comme Adler (1985), la capacité d'attraction de l'artiste dépend de son niveau relatif de notoriété médiatique. Françoise Benhamou souligne, que compte tenu de la grande versatili-

que je sais écrire un livre. Alors je peux me dire, je suis un auteur." [Auteur blogueur] 
té des consommateurs, les producteurs sont confrontés à une forte incertitude sur leur succès ou leur insuccès financier et que l'une des stratégies possibles pour maximiser leur profit, ainsi que le font les producteurs de films par exemple, est d'exploiter la capacité d'attraction détenue par les stars, autrement dit, le star-system.

La situation du livre, au moins pour la plus grande partie des auteurs, est partiellement différente. Pour le sociologue Raymond Boudon (1981), trois marchés s'offrent aux intellectuels pour être reconnus : celui de la certification professionnelle et des audiences spécialisées ; celui du plus large public via des travaux de vulgarisation qui lui sont destinés ; et enfin, celui des médias. On verra plus loin, en étudiant la diffusion, que les médias généralistes n'offrent qu'une porte étroite aux livres, la réservant à quelques rares auteurs; mais que les supports électroniques ont démultiplié les espaces de reconnaissance des auteurs, dans une segmentation qui permet sans doute de retrouver les trois sphères décrites par le sociologue : le public spécialisé (défini par son degré de compétence et son insertion dans les sphères professionnelles), le public concerné (défini par l'intérêt qu'il attache à la thématique), le public médiatique (défini par le support par lequel on le touche).

Par ailleurs, ajoutons que les auteurs peinent à définir ce que serait selon eux une "juste rémunération". La grande majorité, en particulier des auteurs littéraires, s'accorde sur la nécessité d'une rémunération, tout en estimant que le prix des livres est trop élevé et doit être réduit, dût-ce leur rémunération en souffrir.

Il n'y a pas de raison que la musique et les livres soient une sorte de self-service même si on veut que le plus grand nombre nous connaisse. [...] Je pense qu'on a tous à gagner à baisser les prix des livres, à rendre la culture accessible. Pas gratuite parce que sinon on ne vit pas, mais baisser les prix, oui, clairement. [Romancière, Communication, Paris]

On observe dans une moindre mesure chez les auteurs d'ouvrages pratiques et les BDistes que nous avons interviewés le même souci de reconnaissance, ce souci d'exister en tant qu'auteur. En effet, ces auteurs écrivent souvent sur 
des sujets liés à leur métier (ouvrages de nutrition pour le médecin nutritionniste, ouvrages de culture générale pour le professeur de classe préparatoire). Le médecin nutritionniste ne se considère d'ailleurs pas à proprement parler comme un "auteur", mais il associe complètement ses ouvrages à son activité professionnelle.

Mais je garde ma juste place, je ne suis pas écrivain, je suis un vulgarisateur; ça veut dire que les propos qui sont dans mes bouquins ne m'appartiennent pas forcément. Je fais un melting pot de propos que j'ai récoltés et validés scientifiquement. Donc ça me prend du temps certes, mais je n'ai jamais voulu aller au-delà. Je ferais un film ou une pièce de théâtre ce serait différent, mais là ce n'est pas de la littérature, il faut arrêter ! [Nutrition, Paris]

La bande dessinée, elle, semble s'apparenter davantage à une profession (qui nécessite des compétences particulières: par exemple la maîtrise du graphisme ou de la publication assistée par ordinateur), difficilement conciliable avec d'autres activités; et de fait, les auteurs de BD de l'échantillon vivent de cette activité. Si l'envie de reconnaissance et de prestige reste importante chez eux, la dimension statutaire et symbolique de l'auteur l'est moins. Dès lors, la notion de droit d'auteur est souvent immédiatement associée à des considérations très concrètes: la maîtrise du devenir de l'œuvre et le désir d'une rémunération, double position en définitive relativement rare chez les auteurs.

\section{Ambivalence vis-à-vis du piratage}

Les auteurs se considèrent en général comme peu au fait de la diffusion de leurs livres et de leurs différents formats, et le numérique en est un bon exemple: ils ne savent pas la plupart du temps si leur textes ont fait l'objet d'une édition numérique, ou s'ils le savent, estiment être mal informés. A la question de savoir s'il existait une édition pirate de leurs œuvres, la plupart n'ont pas su répondre, s'en souciant peu ou n'imaginant pas que tel pourrait être le cas.

Les attitudes à l'égard du piratage sont caractérisées par l'ambivalence des auteurs : ils souhaitent la diffusion la plus 
large possible de leurs œuvres mais en même temps craignent une perte de contrôle ; ils veulent être lus mais aussi que leur œuvre soit protégée contre un certain nombre d'atteintes; ils acceptent en général la diffusion d'extraits mais sont partagés sur la diffusion globale. Cette ambivalence est d'abord liée à une forme de méconnaissance de l'évolution du numérique, méconnaissance dont on pourrait se demander si elle ne trahit pas avant tout une certaine indifférence à la question du piratage ou du moins une mobilisation faible. L'exemple de la musique, beaucoup plus débattu sur la place publique, apparaît comme une sorte de contre-exemple, surtout parce que le piratage des œuvres conduit à une perte de valeur des contenus :

Le gratuit, non, surtout ne pas tomber dans le syndrome de la musique. La gratuité de la musique par le piratage a eu un effet catastrophique de mon point de vue sur la valeur perçue. Quand j'avais 15 ans j'attendais avec impatience la sortie du prochain album de Dire Straits. Le fait qu'on attendait avec impatience la sortie du CD ou du vinyle faisait qu'il y avait une valeur émotionnelle dans toutes les sorties dont les majors ont certainement abusé d'ailleurs. Mais je trouve que l'effet pervers de la gratuité et aussi de la dématérialisation de la musique fait que c'est devenu, non pas une non valeur, mais une valeur perçue comme hyper faible, ce qui est catastrophique pour les créateurs. [...] Du coup je trouve qu'il faut éviter ça sur le livre, cette dévalorisation. [Romancier, Picardie]

Tout le monde fait ça, même mes enfants, ils font du téléchargement sauvage. Ils n'ont pas conscience de voler quelque chose, c'est du domaine de l'évanescent. [Médecin, Roman policier, Paris]

Cette méconnaissance fait assimiler le piratage à du vol, et non à de la contrefaçon, ce que voudrait la qualification juridique; elle nourrit la revendication, assez banale, d'une taxe levée sur les opérateurs de services ou les fournisseurs d'accès, accusés de favoriser les pratiques pirates ("il faut taxer Google, les FAl, etc. "), tout en se conjuguant en général avec le rejet des lois visant à lutter contre le piratage des contenus. 


\section{f) Plagiat et Contrefaçon}

En France, nous l'avons vu, le droit d'auteur porte sur des œuvres protégées, œuvre au sens de création de forme avec la nécessité qu'elle soit originale. Dès lors, le droit d'auteur ne protège pas intrinsèquement les idées, qui sont dites "de libre parcours ", mais les seules idées qui ont donné lieu à " matérialisation" (un plan ou une maquette par exemple). La jurisprudence a d'ailleurs récemment réaffirmé que "La propriété littéraire et artistique ne protège pas les idées ou les concepts, mais seulement la forme originale sous laquelle ils sont exprimés ${ }^{32}$ ".

Si l'appropriation de l'idée d'un tiers n'est pas sanctionnée par le droit d'auteur, le délit civil et pénal de contrefaçon sanctionne $^{33}$ les atteintes aux droits patrimoniaux, voire au droit moral (sanction civile dans ce dernier cas) de l'auteur sur les $œ u v r e s$ originales mises en forme. Ainsi, pour exemple, les romans qui ont pour sujet l'histoire d'une femme qui est aimée d'un homme mais en préfère un autre sont légion mais ne sont pas pour autant des contrefaçons. Les juges pourront en revanche retenir la contrefaçon lorsque les ressemblances entre deux romans (composition, expression, dialogues, etc.) sont telles que le second roman apparaît effectivement comme une reproduction ou une adaptation de la première œuvre ${ }^{34}$. C'est suivant ces critères qu'il a pu être jugé que "malgré un cadre familial et un thème de départ similaires et au demeurant banal ", le roman La Bicyclette bleue de Régine Deforges ne constitue pas une contrefaçon, même partielle, du roman de Margaret Mitchell Autant en emporte le vent.

Un certain nombre, et cela nourrit sans doute leur faible implication sur le sujet, estime que la bataille est presque perdue d'avance et qu'il est très difficile d'empêcher le piratage.

Sur le principe oui [j'estime qu'une régulation des usages en ligne est nécessaire], et en même temps j'ai le sentiment, surtout en parlant avec des jeunes, que la réponse de l'Hadopi est

32 Cass. 1 ère civ., 17 juin $2003, n^{\circ}$ 04-12.72.

33 Le délit pénal de contrefaçon est puni d'une peine de trois ans d'emprisonnement et 300000 euros d'amende.

34 Cass. 1ère civ., 25 mai 1992, n 90-21.630. 
mal perçue ; et pourtant elle répond à de justes questions ; la difficulté étant d'arriver à ne pas donner l'impression qu'on n'accentue pas le contrôle auquel les jeunes générations sont particulièrement sensibles: les restrictions de l'espace, de la liberté ou de l'anonymat. Et puis, il faut trouver un mode de régulation de la circulation des œuvres immatérielles qui puisse se penser autrement que comme une répression. Pour l'instant, je trouve que les réponses apportées par la loi ne sont pas bonnes; en revanche, il est vrai qu'elle pose de vraies questions ; je ne suis pas du tout pour le laisser-faire non plus. [Homme de lettres, Paris]

En fait, les auteurs sont attachés à la fois à une large diffusion de leurs œuvres et à la reconnaissance du droit d'auteur; quand on leur pose la question du piratage, ils doivent se situer entre ses deux pôles, accordant pour certains plus au droit d'auteur et pour les autres davantage à la diffusion.

Cette tension (ou injonction paradoxale) est bien exprimée par cet auteur qui vit le piratage comme une sorte de dépossession, tout en étant déchiré à l'idée de renoncer aux lecteurs du numérique:

Disons que je sais l'importance que représente le travail d'un auteur. Un livre, c'est un an de boulot! Donc je suis pour le droit d'auteur ! [...]. Pour moi la question de savoir si je passe au numérique ou pas est une torture: aller vers le numérique avec tous les risques que cela comporte ou rester sur le papier et du coup se couper de tous les gens qui me liraient sur iPad, iPhone ou sur Kindle? Mais je suis terrorisé à l'idée de me faire pirater. Est-ce que je vais avoir le choix de toute façon ? C'est très compliqué, mais la douleur de se faire pirater est très simple. Nous, les auteurs de livres, si on n'a pas les revenus des livres, on n'a pas de revenus du tout. On ne peut pas faire de concerts à côté ! On n'a pas de plan B. En plus, c'est terrible de se faire voler quelque chose que l'on a créé soi-même. [Auteur- blogueur]

Les tenants du droit d'auteur soutiennent que " tout travail mérite salaire ", mais tendent surtout à percevoir la rémunération davantage comme une reconnaissance du travail fourni que comme le résultat d'un mécanisme de marché.

Je crois que le droit d'auteur c'est une reconnaissance. C'est la reconnaissance du travail de création. Au-delà de l'aspect 
financier, le fait qu'on parle de droit d'auteur, ça veut dire qu'on reconnaît à l'auteur un droit sur son œuvre. Celui qui crée a besoin de cette reconnaissance. Après qu'il vende cinq mille livres ou deux cents, cela n'a pas d'intérêt en soi, mais il y a quand même cette logique de se dire : j'ai fait passer un concept intellectuel à quelque chose de concret. Donc, c'est important de le défendre. [Premier roman, Paris]

Les motivations des tenants de larges mises en ligne, fut-ce au détriment de la protection de leurs œuvres, sont multiples: diffuser la culture, être lu, favoriser le dialogue... Ils peuvent aussi défendre l'idée d'une éducation publique générale et gratuite :

Le jour où vraiment le monde de l'édition va être piraté à un très très grand nombre, à la limite je me dirais 'chouette', les gens s'intéressent à ce qui est intéressant! [...] Ça voudrait dire que les gens ont envie de connaître. À un moment donné, et là l'éditeur m'a incendiée, je voulais mettre en ligne en consultation gratuite mes ouvrages parce que je considérais que les gens qui avaient envie de s'instruire devaient le faire sans payer, c'est mon côté prof. Mais bon l'éditeur n'était pas d'accord! [Vulgarisation, Paris]

Pour un de nos auteurs qui s'est engagé très largement dans cette démarche d'ouverture par la publication de ses textes sous une licence Creative Commons (qui autorise la diffusion non-commerciale du texte tout en reconnaissant la paternité de l'œuvre), il s'agit aussi de remettre l'auteur à sa place : derrière l'œuvre et non pas devant comme il pense que c'est actuellement trop souvent le cas.

Sur le droit d'auteur, je trouve qu'il y a une mauvaise posture des artistes, des éditeurs, on est vraiment dans un monde de commerciaux qui défendent leurs petits intérêts, pas dans un monde d'amoureux de la musique ou de la littérature. C'est uniquement une affaire de sous. Le statut d'auteur m'énerve beaucoup, j'aurais préféré qu'il n'y ait pas mon nom ou ma photo sur mon livre, on accorde trop d'importance à l'auteur. (...) Je ne sais plus quand Godard avait parlé de ça, il avait dit qu'il fallait arrêter de parler du droit d'auteur mais qu'il fallait parler plutôt des devoirs des auteurs et je suis vraiment d'accord avec lui. [Premier roman, Paris] 
Ce jeune romancier remet en cause non le principe des droits d'auteur mais leur caractère systématique, leur durée et leur transmission :

Je préfère que [mon livre] soit lu surtout, cela ne m'aurait pas spécialement dérangé qu'il soit téléchargé comme ça. Après, s'il se vendait suffisamment pour que je puisse en vivre, peutêtre que cela pourrait me déranger, mais il ne faut pas oublier que c'est seulement quelques dizaines de personnes qui vivent de leurs romans en France ; donc, c'est très très marginal. (...) Souvent quand on entend 'droit d'auteur' on parle de défendre son œuvre, alors que pour moi une fois que l'œuvre est publique, elle n'appartient plus à personne. Quand on entend les héritiers de Picasso qui touchent encore de l'argent, je trouve ça scandaleux, que les gens vivent comme ça de choses qu'ils n'ont pas créées. [Premier roman, Paris]

Cet auteur estime donc que puisqu'il ne vit pas de sa plume, son objectif se résume à être lu, quitte à ce que cela le soit via des moyens illégaux. II souligne par ailleurs le déséquilibre du rapport de force avec l'éditeur qui se manifeste à travers le faible pourcentage perçu par l'auteur sur les ventes de livres comparé à celui reçu par l'éditeur. Ce pourcentage qu'il considère, comme autres auteurs, comme beaucoup plus important, voire excessif. Ce romancier s'inscrit ainsi dans une conception de l'œuvre comme bien public, au sens de bien non rival et qui doit être accessible par tous, et qui in fine n'appartient donc plus à l'auteur.

Á mi-chemin des deux positions, une auteure imagine un système éditorial qui tiendrait à la fois du calendrier du cinéma et d'une réduction massive des prix du livre, proposant la sortie numérique pour une somme symbolique avec un certain différé par rapport à la sortie du livre-papier. Cette auteure propose finalement de transposer le modèle du financement de la production audiovisuelle suivant le principe appelé "chronologie des médias " à la production éditoriale, où l'on séquence en fenêtres de diffusion chaque exposition du film sur des supports distincts : depuis sa sortie en salle, jusqu'à la diffusion gratuite à la télévision, en passant en ordre pré-défini par la VOD (vidéo à la demande) et la télévision payante. Chaque modalité de diffusion permet ainsi d'engranger des nouveaux revenus, et participe au 
financement global de la production cinématographique. Ce nouveau modèle économique ne pourrait-il effectivement pas être envisagé ? II reviendrait à différer dans le temps la sortie d'un ouvrage en proposant par exemple, un ou deux chapitres mis à disposition gratuitement ou pour une somme modique auprès des internautes (sorte de produit d'appel) et ensuite, envisager une sortie en librairie (ou sur Amazon) à un prix différent. Ce système pourrait avoir le mérite d'un nouveau partage de la valeur, qui serait ressenti par les auteurs comme plus équitable, voire plus à leur avantage.

Moi je pense qu'il faut laisser en ligne le livre, je serais tout à fait d'accord pour le faire mais seulement après quelques années, peut-être deux ans; il faut laisser le livre exister quand même. II me semble que symboliquement, il faut qu'il y ait un minimum. De la même manière qu'on a fixé des prix de téléchargement même symboliques, même $1 €$, mais que les gens aient la démarche, parce que la culture est payante à tous points de vue. [Romancière, Communication, Paris]

La diffusion encore faible des liseuses et autres supports de lecture numérique au moment de notre enquête, ajoutée à une faible connaissance des espaces de piratage, explique sans doute pour partie la relative indifférence des auteurs de notre échantillon. L'équipement que l'on annonce rapide en liseuses devrait modifier quelque peu les termes du débat.

\section{Conclusion intermédiaire : le partage de la valeur}

L'attachement à la défense des droits d'auteurs n'est pas directement ou pas seulement lié à leur importance et à leur place dans les revenus de l'auteur. Certains auteurs d'ouvrages pratiques ou BDistes, qui peuvent tirer des revenus significatifs de leurs livres, s'inquiètent, on l'a dit, dans une moindre mesure du piratage. Quelques uns parce qu'ils ressentent un sentiment de paternité moins fort à l'égard de leur œuvre (" moi, je ne fais que compiler et rassembler des connaissances accessibles à tous "). D'autres parce qu'ils n'en vivent pas et donc considèrent qu'ils ne sont pas direc- 
tement affectés par la question. D'autres enfin sont sensibles à l'impact économique qu'ils estiment positif de la diffusion en ligne, prenant position dans un débat très controversé. C'est le cas par exemple de ce bédéiste pour qui il faut faire connaître son travail au grand public (et l'outil Internet remplit très bien cette fonction), de façon à valoriser l'œuvre au maximum ; la mise en ligne d'albums remplit une fonction de communication et de promotion qui met en valeur l'œuvre papier.

Je suis convaincu que les lecteurs veulent propager des trucs qu'ils aiment, ça leur permet de les faire découvrir, comme dans une bibliothèque finalement. Et puis la gratuité elle est partie intégrante d'Internet... Mais cela permet aussi de découvrir des auteurs plus radicaux, gratuitement, comme on feuilletterait dans une bibliothèque. Enfin pour le moment, ce que l'on constate avec mon éditeur, c'est qu'il n'y a pas d'impact négatif sur les ventes avec la mise en ligne gratuite des BD ! Moi j'en tire un enseignement : l'avenir du numérique est dans les formes de Freemium, avec toujours un versant papier qui reste essentiel - en tout cas aujourd'hui - car il n'y a pas de marché numérique. [BD, Paris]

Cet auteur, seul de notre échantillon, se situe dans une perspective semblable à celle des créateurs qui offrent en ligne leurs œuvres pour toucher un plus grand nombre de lecteurs (ou d'auditeurs) et obtenir une rémunération sous la forme de notoriété, faisant l'hypothèse que cette forme de diffusion, loin de nuire à la déclinaison payante de leur travail, la sert au contraire et la conforte. Cette conception peut être qualifiée d'externalités de consommation, au sens où l'utilité du service artistique est fonction croissante de sa qualité intrinsèque, mais également du nombre d'individus qui le consomment, générant des discussions ou encore le sentiment d'appartenance à un groupe. On parlera alors "d'influence normative ".

En plus mineur, se pose également pour les auteurs la question de la rémunération et du partage de la valeur avec la diffusion de leurs livres sur Internet. La question est controversée et divise beaucoup les auteurs: le plus grand nombre se pose des questions sur le différentiel de rémunération entre livre en ligne, livre numérique, livre papier... 
Certains revendiquent des droits d'auteur plus importants, puisque les coûts de fabrication diminuent.

Je n'écris pas pour vivre mais par passion. Donc je trouve qu'il faut défendre la rémunération des auteurs, mais elle ne sera jamais à la hauteur du travail qu'on fait, c'est un travail de titan, mais je ne saurais pas tellement répondre à cette question. Je crois qu'il y a eu des expériences de gens qui s'éditent en ligne, donc qui "squeeze " la distribution et qui du coup touchent plus d'argent. Je trouve que c'est une expérience intéressante, mais je ne me suis jamais personnellement inscrit dans cette trajectoire. Je trouve que c'est normal d'être rémunéré, mais il y a toute une chaîne qui doit être respectée, le diffuseur, le libraire ont un rôle et je n'ai pas l'impression que ces gens-là roulent sur l'or. Je ne crois pas qu'ils gagnent énormément d'argent. Peut-être que le numérique va permettre une redistribution différente, je ne sais pas. [Romancier, Picardie]

II y a un phénomène qui joue considérablement, entre le physique et le numérique, c'est bien évidemment toute la chaîne de distribution qui saute. Donc à partir de là, on peut faire une marge plus grande pour l'auteur et pour l'éditeur. En résumé un livre que l'on vend 15 euros aujourd'hui, sur lequel l'auteur gagne 1,5 euro, en gros, eh bien on pourrait très bien le vendre 10 euros en publication numérique et donner 4 euros à l'auteur... Donc diminuer le prix de vente tout en augmentant les droits perçus par l'auteur. Ce serait plus intelligent, et le schéma idéal. Mais ça ne se fera jamais. L'auteur peut toujours aller faire ses carreaux chez lui, c'est lui qui bosse... Mais il ne gagne rien. Je n'y crois pas une seule seconde. [Romancière, Communication, Paris]

Notre romancier, comme plusieurs autres, trouve ici la martingale qui donnerait à l'auteur la clef pour sortir de son ambivalence, un système qui à la fois le rémunèrerait mieux et augmenterait le nombre de ses lecteurs en abaissant les prix de vente de ses ouvrages. Mais comme on le sait, les martingales augmentent plus l'espérance du joueur que ses gains! La numérisation des ouvrages n'aboutit pas à une désintermédiation, mais à une réintermédiation qui voit naître de nouveaux intermédiaires et de nouvelles pra- 
106 | Internet dans la diffusion, la diffusion hors Internet

tiques ${ }^{35}$. C'est que nous allons maintenant étudier en nous penchant sur le premier intermédiaire de l'auteur, l'éditeur.

35 Voir la brochure de l'Hadopi, Au fil des Labs \# 1, décembre 2011. 


\section{Les relations Auteurs - Éditeurs à I'ère du numérique}

Comment les auteurs voient-ils leurs relations avec les éditeurs ? II s'agira en particulier d'évaluer dans quelle mesure le numérique fait évoluer ces liens et le rôle de l'éditeur dans la filière du livre. Nous verrons que l'ambivalence est présente à plusieurs niveaux dans le discours de l'auteur, et qu'elle tient principalement au double rôle joué par la maison d'édition: à la fois première reconnaissance du travail de l'auteur, soutien et conseil dans la création de son $œ u v r e$, éventuellement source de prestige sur le plan symbolique, mais aussi clef d'entrée pour l'exploitation et la commercialisation du livre. A ce titre, l'éditeur est simultanément pour l'auteur un "objet de désir " et une source de craintes, un facilitateur et un décideur économique. C'est lui qui permet à l'auteur d'actualiser son projet d'écriture et de lui donner une forme aboutie, mais c'est aussi lui qui possède la maitrise de la diffusion, le lien avec l'attaché de presse, le format du livre et sa maquette, son prix, parfois son contenu. Dans ce contexte, le numérique pourrait offrir de nouvelles possibilités en ouvrant des capacités éditoriales originales; cependant, comme on le verra, il s'agit encore d'alternatives relativement marginales, non seulement parce qu'elles apparaissent, à tort ou à raison, comme faiblement répandues et assez exclusives, mais aussi parce qu'elles sont peu valorisées par les auteurs. 


\section{g) Quelques éléments juridiques clés du Droit d'auteur}

Le droit d'auteur est l'ensemble des prérogatives exclusives dont dispose un auteur (ou un groupe de co-auteurs) sur ses (ou leurs) œuvres de l'esprit. Le droit d'auteur comprend des prérogatives d'ordre patrimonial et d'ordre extrapatrimonial.

Les droits patrimoniaux confèrent un monopole d'exploitation économique sur l'œuvre, pour une durée variable, selon les pays ou les situations, au terme de laquelle, l'œuvre entre dans le domaine public. En France, ce terme est de soixante-dix ans après le 1er janvier suivant le décès de l'auteur. S'ajoute au monopole d'exploitation, dans le domaine des arts graphiques et plastiques, un "droit de suite ", qui permet à l'auteur et à ses héritiers de percevoir un pourcentage sur les ventes publiques successives des objets d'art.

Le droit moral est une spécificité de la conception française du droit d'auteur aujourd'hui consacrée à l'échelon international. C'est un droit extrapatrimonial, perpétuel, imprescriptible et inaliénable, attaché à la personne de l'auteur, et qui tend à conserver et à défendre l'esprit de l'œuvre. Le droit moral peut être exercé par l'auteur lui-même ou par ses ayants droit, sans limitation de durée. Le droit moral comprend quatre attributs :

$1^{\circ}$ le droit de divulgation ${ }^{36}$, droit de communiquer l'œuvre au public est décidé par l'auteur seul, dans des conditions qu'il aura choisies ;

$2^{\circ}$ le droit de paternité 37 ou droit au respect du nom, l'auteur a droit au respect du lien de filiation entre lui et son œuvre ;

$3^{\circ}$ le droit au respect ${ }^{38}$ permet à l'auteur de faire respecter l'intégrité de son œuvre, tant matérielle que spirituelle ;

$4^{\circ}$ le droit de retrait et de repentir ${ }^{39}$, permet à l'auteur, sous réserve du respect de certaines conditions, de retirer son œuvre du circuit commercial, même après sa divulgation.

Il convient de noter que la protection d'une œuvre de l'esprit par le droit d'auteur n'est pas conditionnée par un dépôt préa-

\footnotetext{
${ }^{36}$ Article L.121-2 du code de la propriété intellectuelle (CPI)

${ }^{37}$ Article L.121-1 du CPI

38 Article L.121-1 du CPI

39 Article L.121-4 du CPI
} 
lable. Les œuvres sont en effet protégées du seul fait de leur matérialisation, même inachevée, dès lors qu'elles revêtent un caractère original. Ainsi, "L'auteur d'une œuvre de l'esprit jouit sur cette œuvre, du seul fait de sa création, d'un droit de propriété incorporelle exclusif et opposable à tous. " ${ }^{40}$. En outre, "l'œuvre est réputée créée, indépendamment de toute divulgation publique, du seul fait de la réalisation, même inachevée, de la conception de l'auteur. ${ }^{41}$

Toutefois, des auteurs recourent à l'envoi recommandé à euxmêmes ou au dépôt via les sociétés de gestion collective et autres associations ou syndicats représentant les auteurs, qui proposent souvent ce service à leurs membres. Si le dépôt est présenté comme un des moyens de dater une œuvre ou de prouver sa qualité d'auteur, il est toutefois d'une faible valeur probante - voire irrecevable en justice.

\subsection{La recherche par l'auteur d'un "partenaire"}

Les auteurs que nous avons interviewés jugent de manière assez variable le travail de leur éditeur. Notons que l'enthousiasme est assez rare et les critiques virulentes ne sont pas exceptionnelles. L'enquête de la SCAM, "Relations auteurs / éditeurs " 42 , je t'aime moi non plus ", ne disait pas autre chose, faisant état d'un taux global de satisfaction de $60 \%$ et de $9 \%$ de situations conflictuelles alors même que les auteurs interrogés faisaient partie de la frange professionnalisée ${ }^{43}$.

Toutefois, la grande majorité d'entre eux n'envisage pas de se séparer de leur éditeur étant donné son rôle déterminant dans l'accès à la filière du livre. Enfin, et nous le verrons par la suite, si l'éditeur permet l'accès aux canaux de diffusion et de distribution du livre, il est d'abord et avant tout un

\footnotetext{
40 Article L.111-1 du CPI

${ }^{41}$ Article L.112-2 du CPI

42 www.scam.fr/fr/Actualit\%C3\%A9s/lesdossiers/relationsauteursediteurs/Jetai memoinonplus/tabid/363519/Default.aspx

43 Recevant par exemple pour les deux tiers d'entre eux des à-valoir.
} 
gatekeepers ${ }^{44}$, parce qu'il confère pratiquement et symboliquement le statut d'auteur en acceptant de publier un manuscrit. C'est véritablement à partir du moment où il est publié que l'auteur assoit son statut, qu'il devient à proprement parler un auteur.

Et les auteurs savent bien que le rapport à l'éditeur est, pour la grande majorité d'entre eux, asymétrique :

Franchement, je me suis senti bien chez [cette éditrice], j'ai regardé le contrat en diagonale, je l'ai signé et voilà. En toute honnêteté, il y a trois mille manuscrits envoyés par des auteurs pour un seul qui sera édité ; je n'avais pas envie de faire ma diva, j'étais déjà extrêmement content d'être édité. [Romancier, Picardie]

En triant, en sélectionnant parmi les très nombreux manuscrits qu'il reçoit, ceux qui méritent d'être publiés ou non, l'éditeur joue un rôle de label, de marque ; il apporte une garantie de la qualité du livre auprès des lecteurs et incarne la fonction de médiation entre l'auteur et le grand public. Ce rôle inclut de ne pas forcément "coller " complètement aux attentes du public et d'avoir la capacité de prendre des risques, pour proposer des œuvres nouvelles et originales.

C'est à ça que sert l'éditeur : à faire reconnaître, à estampiller... et à ça que sert le prix du livre... L'éditeur, je l'ai bien vu, parce que moi j'étais le premier à publier un roman chez lui, alors ils ont pris tous les risque avec moi... c'est un travail de détection, de prise de risque, et d'accompagnement du livre... Internet ne pourra pas supprimer les éditeurs... [Jeune auteur, Paris]

Sans doute faudrait-il nuancer le prestige et la reconnaissance que la maison d'édition apporte à l'auteur ; à côté des grandes maisons, existent une très large multiplicité d'éditeurs de moindre notoriété, qui constituent souvent pour les auteurs une alternative intéressante dès lors qu'ils sont novices, peu connus ou faiblement soutenus :

Oui, [on cible les éditeurs auxquels on envoie notre manuscrit] selon ce qu'ils publient, ce que les auteurs peuvent dire d'eux,

${ }^{44} \mathrm{Ce}$ terme désigne un acteur clef permettant l'accès à un marché spécifique, en l'occurrence, ici, celui du livre. 
donner ou pas envie d'être édité par ces personnes-là. Mais bon après, d'abord on envoie aux plus gros, aux plus prestigieux. Comme tout le monde, on est d'abord refusé par Gallimard, par Grasset... [Premier roman, Paris]

L'étape de démarchage des éditeurs en vue d'une publication relève parfois d'un véritable " passage à l'acte ", source d'appréhension, notamment pour les auteurs débutants. Dans le même temps ce moment est initiatique : il les propulse dans un autre champ, celui de l'édition, qui ne concerne plus la création proprement dite, mais la diffusion, la commercialisation et le devenir de leur œuvre. La publication peut d'ailleurs constituer l'aboutissement d'un parcours fastidieux de démarchage. Si certains manuscrits rencontrent très rapidement un éditeur, d'autres ne sont publiés que plusieurs années après avoir été écrits, parfois à la suite de nombreux remaniements.

J'en ai écrit sept en tout, et j'en ai envoyé deux seulement, un qui a été accepté et l'autre qui a été refusé. Les autres je ne les avais pas envoyés, je ne me sentais pas prêt. [Romancier, Picardie]

En fait je l'avais envoyé à trois éditeurs, et j'ai reçu deux refus après, mais comme j'ai reçu les refus après le feu vert je n'ai pas eu le temps de déprimer ou de m'angoisser, c'est allé très vite parce qu'ils ont reçu le manuscrit fin janvier et le livre est sorti début mai ! [Romancière, Communication, Paris]

Dans les faits, l'envoi du manuscrit aux maisons d'édition obéit à une démarche plus ou moins raisonnée. Les auteurs débutants tendent à multiplier les envois ou, ce qui s'avère selon eux souvent plus efficace, à faire jouer leur réseau relationnel. Le hasard des rencontres et des connaissances ayant un lien avec le milieu de l'édition joue souvent un rôle important, notamment, pour le premier ouvrage : cela peut constituer l'élément déclencheur pour la publication.

J'avais envoyé à une dizaine de maisons et j'ai eu deux pistes sérieuses. [Romancier, Picardie]

Je cherchais très mollement un éditeur, ce n'était pas un projet au départ. Je voulais avant tout construire un roman qui tienne debout. Au deux tiers du trajet, j'ai rencontré $X$ en personne, par le biais professionnel [à son cabinet médical]. II y a beaucoup de gens de type artistes dans le [quartier]. Je lui en ai 
parlé et il a accepté d'étudier le manuscrit, c'était amical. [Médecin, Roman policier, Paris]

Comment j'ai commencé ? [...] J'ai eu une discussion avec un copain qui m'a dit pourquoi n'écrirais-tu pas en décrivant la police telle que tu aimerais la faire connaître ?' [...] J'ai écrit une nouvelle d'une quarantaine de pages [...] Cette nouvelle je la fais lire à mon camarade et il la fait lire à un producteur [...] Quelques jours plus tard, ce producteur demande à me rencontrer. On se retrouve assis dans son bureau. Et il me dit "tu as écrit ça? On va en faire un film." [Policier, Roman policier, Paris]

Beaucoup se font introduire par l'intermédiaire de relations auprès d'un éditeur :

En fait, il fallait que je trouve la porte d'entrée auprès des maisons d'édition. J'ai un jeune frère qui à l'époque travaillait au musée de $\mathrm{X}$ et dont la femme était dans la communication; c'est une journaliste freelance qui a beaucoup de talent pour écrire des bouquins dans un style un peu enlevé. Elle m'a dit qu'elle allait chercher des éditeurs. Moi je lui ai apporté le projet, je voulais faire un bouquin pour les adolescents sous forme de questions réponses. Elle a donc cherché des éditeurs et abordé $Y$, qui était intéressé et qui nous a trouvé une illustratrice que, en plus, j'ai trouvé très pertinente. [Nutrition, Paris]

Les auteurs les plus expérimentés adoptent quant à eux plus fréquemment une stratégie de ciblage des maisons : ils s'adressent avant tout aux éditeurs dont le positionnement éditorial correspond le mieux à leur œuvre, type d'ouvrage, style d'écriture, collection notamment. Une telle stratégie augmente à la fois les chances de publication et favorise une meilleure promotion par un éditeur connaissant bien les leviers sur lesquels agir, pour un genre de livre qu'il a I'habitude de publier.

Par exemple, pour ce genre d'ouvrages de vulgarisation, il n'y avait pas mieux que cet éditeur, on ne pouvait pas le présenter à Vuibert ou Masson. II faut voir ce que fait l'éditeur, et il faut s'adapter à la collection qui est choisie, au style et autre. Parce qu'ensuite, vous êtes sûr d'avoir des lecteurs qui vont plutôt se diriger vers un type de travail ou vers un autre. [Vulgarisation, Paris] 
Cette recherche d'un partenaire adapté peut conduire l'auteur à multiplier ses éditeurs en fonction des différents types d'ouvrages qu'il veut publier; et tous les auteurs interviewés ont expliqué n'avoir connu aucun problème de droit de suite, avec leur éditeur, quel qu'il soit, dès lors qu'il s'agissait d'un genre différent :

[Mes éditeurs spécialistes de littérature enfantine] m'ont laissé partir pour les romans, ça s'est très bien passé bien qu'ils aient eu un droit de préférence... D'elle-même la directrice m'avait dit, 'on n'est pas capable de le défendre', parce que comme ils n'en faisaient quasiment pas, c'est difficile d'avoir du pouvoir, du poids pour cette littérature, de la crédibilité face aux libraires qui sont quand même le maillon incontournable et fort de la chaîne. Quand un éditeur sort trois romans par an, il n'a pas de poids. [Romancière, Communication, Paris]

Comme les éditeurs, les auteurs spécialisent leur partenariat et l'adaptent au type d'ouvrage qu'ils cherchent à publier. Si nombre de nos auteurs ont évoqué des conflits avec leurs éditeurs, cela n'a jamais été lié à un ouvrage qu'ils auraient refusé de lui confier ; et lorsque c'est l'éditeur qui l'a refusé, ils le mettent sur le compte d'une relation dégradée plus qu'ils ne l'attribuent à un changement de genre ou de style.

\section{Comment établir la confiance}

Au-delà des stratégies exposées, le choix de la maison d'édition dépend des premières rencontres entre éditeur et auteur, de la relation qui s'établit avec le responsable dès l'abord, mais aussi dans la durée. Tous les auteurs soulignent à quel point l'écriture d'un livre requiert un travail exigeant et un investissement personnel majeur. Dès lors, leurs attentes à l'égard de l'éditeur (ou le directeur de collection pour les maisons d'édition de tailles plus importantes), son implication, son enthousiasme, la qualité de son jugement et la confiance qu'il leur inspire, sont elles aussi très élevées. Beaucoup des auteurs interviewés recherchent dans l'éditeur à la fois un soutien, une personne qui apprécie leur travail et un véritable "partenaire " qui saura les accompagner et les guider dans toutes les étapes 
du processus, depuis "l'accouchement " de l'œuvre jusqu'à sa commercialisation. Les auteurs, et notamment les romanciers dont l'implication et le sentiment de paternité visà-vis de leur œuvre sont sans doute les plus forts (tandis que les auteurs d'ouvrages pratiques et les BDistes ont souvent un rapport plus distancé à l'égard de leurs ouvrages) décrivent ce qu'ils attendent des rapports avec leur éditeur en des termes à la fois très forts et teintés de beaucoup d'affect. Le ressenti intuitu personae apparaît comme un critère d'appréciation essentiel de l'éditeur.

Alors, qu'est-ce que j'en attends... Déjà, qu'il me dise "j'aime " et que ce soit sincère, alors on peut parler. (...) Les deux fois que j'ai quitté un éditeur, c'était à cause d'une mésentente. Soit l'éditeur t'accompagne dans ton univers, soit on passe à autre chose, chez un autre éditeur. [Littérateur, Paris]

Maintenant j'ai un vrai rapport de fidélité avec cet éditeur, tout se passe bien, donc s'il continue de prendre mes textes, a priori... [Romancier, Paris]

Et après une rencontre avec cette personne, j'ai eu un 'coup de foudre' et me suis senti très bien avec cette maison. [Romancier, Picardie]

C'est une relation humaine et de confiance avec son éditeur, d'empathie, on apprend à se voir, à s'écouter comme avec les patients. J'aime bien savoir à qui j'ai à faire, savoir si on s'entend bien, s'il va défendre mon bouquin, etc... Et ça ce n'est pas Internet qui va me l'apprendre, ce n'est pas Internet qui va me l'apporter. [Nutrition, Paris]

Pour d'autres auteurs plus expérimentés, le caractère nonintrusif de l'éditeur est apprécié. Sont alors d'abord mis en avant la qualité de l'accompagnement, le soutien et non pas l'intervention de l'éditeur dans le manuscrit. Cette primauté de l'affect peut être facteur de déceptions, de conflits et de frustrations. Les auteurs de livres pratiques sont davantage sur un mode d'échange: ils ne recherchent pas une forme de paternalisme, mais plutôt un avis expert dans la chaîne de production et de diffusion du livre.

Nous verrons par la suite que si le niveau d'exigence des auteurs est très élevé, à la hauteur du travail qu'eux-mêmes fournissent, les rapports réels avec l'éditeur peuvent être 
source de déception, voire de désillusion. En l'occurrence les jeunes auteurs qui ont eu peu de latitude dans le choix de leur maison d'édition sont particulièrement sujets à ce genre de frustrations. De fait, le rôle de l'éditeur dans le travail de création est diversement apprécié, voire attendu, par les auteurs.

\section{h) Le droit de suite}

De nombreux auteurs évoquent un "droit de suite " contractuel qui les obligerait à présenter prioritairement à leur éditeur un certain nombre d'œuvres pendant une période donnée.

Le droit d'auteur n'utilise pas cette terminologie:

Le droit de suite, tel que le dénomme la loi ${ }^{45}$, permet aux auteurs d'œuvres graphiques ou plastiques (tableau, sculpture, etc.) d'être intéressés aux ventes publiques successives des objets d'art dont ils sont les auteurs.

Le droit de suite " évoqué par les auteurs, mais différent de l'acception légale, est un pacte de préférence aux termes du code de la propriété intellectuelle 46, qui est une promesse faite par l'auteur à son éditeur, par laquelle il s'engage contractuellement à proposer cinq ouvrages, d'un genre défini et pour une durée de cinq années.

L'article qui régit ce pacte de préférence est le suivant : "Est licite la stipulation par laquelle l'auteur s'engage à accorder un droit de préférence à un éditeur pour l'édition de ses œuvres futures de genres nettement déterminés. Ce droit est limité pour chaque genre à cinq ouvrages nouveaux à compter du jour de la signature du contrat d'édition conclu pour la première œuvre ou à la production de l'auteur réalisée dans un délai de cinq années à compter du même jour. L'éditeur doit exercer le droit qui lui est reconnu en faisant connaître par écrit sa décision à l'auteur, dans le délai de trois mois à dater du jour de la remise par celui-ci de chaque manuscrit définitif. Lorsque l'éditeur bénéficiant du droit de préférence aura refusé successivement deux ouvrages nouveaux présentés par l'auteur dans le genre déterminé au contrat, l'auteur pourra 
reprendre immédiatement et de plein droit sa liberté quant aux $œ u v r e s$ futures qu'il produira dans ce genre. II devra toutefois, au cas où il aurait reçu ses œuvres futures des avances du premier éditeur, effectuer préalablement le remboursement de celles-ci."

\section{Un soutien plus ou moins investi dans le travail de création}

L'éditeur représente d'abord une personne de confiance, un soutien dans le travail de l'auteur. Ses remarques sur le manuscrit et son avis sont souvent déterminants dans certains choix importants : c'est le cas pour le choix du titre par exemple. Le degré d'intervention de l'éditeur sur le manuscrit est cependant très variable, selon les auteurs, leur maturité, leurs relations.

Sur le principe, la plupart des auteurs considèrent que l'éditeur (ou le directeur de collection) a un véritable rôle à jouer dans le processus de création. Ils attendent de ce dernier un regard critique et distancié sur leur œuvre, à la fois une vision d'ensemble, et une attention au détail. L'éditeur doit apparaître comme un véritable "accoucheur" de l'auteur, pour reprendre les propres termes de l'un des interviewés, et l'aider à se dépasser.

Aux États-Unis, ils distinguent l'editing du publishing. Ce sont en effet des métiers propres. Mon éditeur il doit savoir être un bon publisher: savoir imprimer et bien diffuser, s'assurer des stocks, d'une bonne couverture... Mais il doit aussi faire un bon editing, c'est-à-dire aider à l'accouchement de l'œuvre. Je crois que Maurice Benhamou parlait de maïeutique... Ils ont pourtant de moins en moins le temps de le faire bien. On attend de l'aide en tant qu'auteur, surtout au début pour éviter les erreurs. Mais ça semble se perdre. [BD, Paris]

L'auteur apporte une pierre mais c'est l'éditeur qui va finir la statue. Elle peut être déjà très bonne mais c'est là que vient l'habileté de l'éditeur à la rendre parfaite et terminée. C'est un métier, de voir à quoi l'auteur visait dans son travail, mais puisque l'auteur a du mal à s'écarter et voir l'ensemble de son travail, là est le moment où l'éditeur donne la valeur ajoutée. [Jeune auteur, Paris] 
L'éditeur peut conseiller l'auteur sur la forme: améliorer la formulation des phrases, trouver un terme qui exprime mieux la pensée de l'auteur, raccourcir certaines parties; mais aussi sur le fond : l'intrigue, le contexte, les dialogues... Plusieurs insistent cependant sur le refus que leur œuvre ne soit dénaturée par des modifications trop importantes. Ils préfèrent accentuer leurs efforts afin de proposer à l'éditeur un manuscrit le plus achevé possible, qui demandera peu de re-travail.

Enlever des choses sur lesquelles je me suis un peu trop étendu, des choses comme ça. Changer les phrases ça arrive dans certains cas, mais je travaille beaucoup mon manuscrit avant de le rendre. [Jeune auteur, Paris]

Par exemple il y a un livre où j'avais commencé en expliquant le contexte de la famille [...] et au début j'avais commencé en expliquant qui est qui au plus-que-parfait. Et elle m'avait dit “c'est dommage d'utiliser le plus que parfait pour commencer le livre, c'est une comédie, ce serait mieux d'utiliser du présent... [Romancière, Communication, Paris]

$\mathrm{X}$ me donnait pas mal de conseils de narration, mettre telle planche à tel endroit, c'était des petites histoires indépendantes en une page, donc on avait vu l'ordre, on avait vu la manière de travailler sur la bichromie. On travaillait les couleurs, l'agencement du livre. Après, quand je bossais avec $\mathrm{X}$, c'était autre chose, c'était plus, par exemple: "attention, là dans votre récit il y a un peu trop de rêve ", c'était un peu plus général. Et, pour le reste, il nous laissait faire. [BD, Bretagne]

Le choix du titre du livre et celui de sa couverture sont généralement du ressort de l'éditeur, même si l'opinion de l'auteur reste importante. Malgré quelques désaccords, les auteurs que nous avons rencontrés considèrent pour la plupart en effet que titre et couverture font partie de la promotion des livres et que l'avis de l'éditeur est souvent plus éclairé que le leur. Des recommandations pertinentes en la matière de la part de la maison d'édition participent donc de sa valeur ajoutée.

Un titre, une quatrième de couverture, une couverture, j'estime que cela ne m'appartient plus, ça appartient au domaine de la réception et donc j'estime que je suis trop dedans. [Littérature enfantine, Paris] 
Dans les faits toutefois, le degré d'intervention de l'éditeur sur le manuscrit est très variable. II tend à être plus marqué auprès des auteurs débutants, qui semblent également davantage en attente de réassurance et de conseils.

Pour moi, avec les éditions $X$, ça se passe très bien parce qu'il y a une très forte proximité, l'auteur est vraiment accompagné, on n'est pas laissé tout seul même si pour l'instant mon expérience est courte, je n'ai qu'un seul roman. Mais une fois remis le manuscrit, on travaille avec l'éditeur sur le texte, pour éventuellement réorienter certains petits passages. [Romancier, Picardie]

Observons toutefois, même à partir d'un échantillon limité d'auteurs, que la participation de l'éditeur varie beaucoup selon la maison d'édition et les ressources en personnel et en temps qu'elle a la possibilité de dédier à l'auteur. Plusieurs estiment d'ailleurs que ces maisons consacrent de moins en moins de temps au re-travail du manuscrit et regrettent ce manque d'implication de l'éditeur, alors qu'ils jugent sa fonction essentielle.

[J'attends] qu'il m'aide à rédiger des phrases, me donne des idées, que ce soit collégial. Mais ce n'est pas le cas : en général ils sont super à la bourre. [Médecin, Roman policier, Paris]

En général, l'éditeur, moins il peut travailler, mieux c'est. Donc c'est vrai qu'il aime quand le texte est propre, mais c'est dommage, parce que c'est un vrai travail, l'éditeur, c'est un vrai métier, c'est à la fois une question d'apprentissage et d'expérience, un jeune éditeur est moins bon qu'un vieux à faire ce travail qui est tout à fait complémentaire de celui de l'auteur. [Jeune auteur, Paris]

\section{L'éditeur comme médiateur}

Pour tous les auteurs, en dehors du soutien dans le travail d'écriture, l'autre mission essentielle de la maison d'édition est la promotion des livres auprès des réseaux de distribution et des différents canaux de communication, que nous avons déjà partiellement évoquée dans le deuxième chapitre. Cette promotion par la maison d'édition reste un motif d'insatisfaction majeur chez bon nombre d'auteurs. Ils regrettent le manque d'efforts consacrés à la promotion de 
leur livre, particulièrement délaissée par les éditeurs sur Internet.

En filigrane, se dessine dans leurs propos, le mode de fonctionnement du marché de l'édition avec une abondance de livres qui doivent atteindre un maximum de ventes en un temps limité. En effet, la plupart des livres restent très peu de temps sur les tables des libraires et sont vite remplacés par des nouveautés, ce qui nécessite donc une promotion rapide pour être efficace. II existe donc un décalage important entre le temps court de la promotion et le temps que l'auteur a lui-même consacré à écrire son ouvrage, et ce décalage s'avère pour nos auteurs facteur de frustrations, d'incompréhensions. Les stratégies éditoriales évolueraient non plus dans le but de sélectionner les grands auteurs de demain mais plutôt les tirages d'aujourd'hui, en d'autres termes vendre moins de titres mais d'avantage d'exemplaires (Benhamou, 2003).

Au plan commercial, j'ai l'impression ils ne font pas tout ce qu'ils peuvent pour vendre les livres. J'ai une grosse promo mais après rien. J'ai l'impression que le bouquin n'est pas visible sur les points de vente. C'est un grand mystère. II faut dire que c'est la foire d'empoigne pour mettre son livre sur la table du point de vente. [Médecin, Roman policier, Paris]

Pour mon dernier livre non, on est sorti et on n'a pas eu un seul article de presse. Rien n'a été fait. Je n'ai jamais vu ça, pourtant on a vendu presque 500000 exemplaires du précédent. La promotion s'est faite par le bouche-à-oreille comme ça, mais du service de presse, rien du tout. Pourtant c'est quand même aussi leur intérêt normalement. Je ne comprends pas. [Vulgarisation, Paris]

Les reproches se concentrent très souvent sur l'attaché de presse de la maison d'édition, la face la plus visible de la promotion pour l'auteur.

Ce que j'ai reproché et que je reproche toujours à [tel éditeur] c'est qu'ils ont une attachée de presse qui à mon avis est une catastrophe complète : je mets les pieds dans le plat parce que je suis content de tout, sauf de l'attachée de presse. Une fois elle m'a fait aller à une conférence où il y avait trois péquins; donc j'ai perdu une journée entière et elle ne s'est même pas excusée. [Nutrition, Paris] 
D'autres tissent des liens étroits avec leur attaché de presse.

L'attachée de presse, c'est un peu ma maman. Elle est là pour faire l'interface avec un univers que je ne connais pas du tout, qui est celui des journalistes. C'est elle qui va faire en sorte qu'il y ait des papiers, qu'il se passe quelque chose autour du livre, etc. [Littérateur, Paris]

Jugements négatifs comme positifs sur les responsables des relations de presse témoignent d'une même attente de prise en charge face à un univers dont les auteurs estiment ne pas avoir les clefs. Certains compensent, on l'a vu, ce qu'ils estiment être la carence de leur éditeur en organisant euxmêmes leur promotion sur Internet ou auprès de leurs propres contacts dans les médias.

Il avait un attaché de presse qui a rempli son rôle malgré tout, mais il a d'ailleurs reconnu que les retombées étaient plus de mon côté, par mes 'réseaux'. [Nutrition, Paris]

Comme on le lit dans ces propos, les attentes des auteurs envers leur(s) éditeur(s) restent donc extrêmement élevées. Une enquête plus large nous permettrait d'établir une sorte de typologie des éditeurs vus par les auteurs à partir de la manière dont ils répondent précisément à ces attentes: soutien à la création, soutien à la mise au point du manuscrit, soutien à sa diffusion, production d'un ouvrage de qualité... II serait tout particulièrement intéressant de comparer la manière dont sont perçus les " petits " éditeurs, par exemple les éditeurs régionaux, les éditeurs spécialisés, les éditeurs à petit catalogue: nos auteurs semblent signaler que cette taille réduite permet des échanges plus étroits, un suivi plus proche, une appropriation plus forte de l'ouvrage par l'éditeur au prix toutefois d'investissements moindres dans la diffusion du livre, sa mise en place, le suivi personnalisé de l'auteur...

\subsection{Des relations contractuelles}

La relation entre l'éditeur et l'auteur est principalement formalisée par un contrat ; disons tout de suite que cet aspect importe assez peu à la grande majorité de nos auteurs. 
Certes, tous ont reçu un tel document mais ils s'y intéressent d'une manière pour le moins distraite, n'en connaissent que très grossièrement les clauses, ont retenu quelques rares éléments, comme par exemple, le montant des à-valoir, s'il y en a, le pourcentage sur les ventes - avec une certaine imprécision - ou encore, éventuellement, le nombre d'exemplaires remis. Cela ne les empêche pas pour autant comme nous le verrons, d'exprimer un avis sur ce contrat qui les lie à leur éditeur.

\section{i) Étude de la SCAM sur la rémunération des auteurs par les éditeurs ${ }^{47}$}

-Dans $80 \%$ des cas (84\% en 2009) les droits d'auteur sont calculés sur le prix de vente public HT conformément au Code de la propriété intellectuelle (article L131-4 du CPI). $13 \%$ sont rémunérés au forfait (11\% en 2009) et $4 \%$ sont rémunérés sur le prix de vente en gros, en totale infraction avec le Code de la propriété intellectuelle.

$-75 \%$ des auteurs perçoivent entre $5 \%$ et $12 \%$ du prix de vente, ils étaient $70 \%$ en 2009 ; mais cette augmentation est due à une baisse du nombre d'auteurs percevant plus de $12 \%$ du prix de vente (ils sont $7 \%$ en 2010 contre $10 \%$ en 2009).

-Si $68 \%$ des contrats comportent un à-valoir (proportion en légère augmentation), l'écart entre les montants se creuse. Ainsi, les à-valoir supérieurs à 3000 euros représentent $37 \%$ des cas (32\% en 2009), et les à-valoir inférieurs à 1500 euros représentent désormais $30 \%$ des cas (25\% en 2009).

-Un auteur sur deux ne négocie pas le taux proposé par l'éditeur (proportion stable).

\section{Les droits directs}

Même lorsqu'ils constituent une part importante de leurs revenus, les auteurs déclarent n'avoir qu'une idée très vague du montant de leurs droits, y compris pour ceux touchant des à-valoir ; le sentiment dominant étant que l'auteur

47 Baromètre 2010 des relations Auteurs / Éditeurs. 
est le bout de la chaîne, faiblement rémunéré par rapport au travail fourni.

- Vous connaissez votre pourcentage?

- J'ai oublié, mais ce n'est pas énorme. Ce qui m'a empêché de m'y intéresser, c'est que grosso modo, le distributeur touche, le diffuseur, l'éditeur puis enfin l'auteur; donc l'auteur touche $3 \%, 4 \%, 5 \%$, ce n'est pas énorme. [Romancier, Picardie]

La réponse la plus fréquente à une question sur les droits directs prend la forme d'un pourcentage, souvent dans une large fourchette, qui peut s'étendre de 3 à $5 \%$ [Romancier, Picardie] à un taux fort élevé au regard des chiffres fournis par la SCAM qui oscille entre 8 et $14 \%$ [Romancière, Communication, Paris]. Seuls, les auteurs faisant l'objet de tirages importants évoquent l'existence d'une échelle qui lie le pourcentage des droits au nombre d'exemplaires vendus.

Les droits d'auteur ont ainsi une signification avant tout symbolique. Pour la quasi-totalité des auteurs, ils représentent une forme de reconnaissance du travail fourni (" tout travail mérite salaire "). Si le discours de l'écriture-vocation est présent...

L'intérêt, c'est de faire le livre, le plaisir qu'on a pris à le faire, ce qui suit après c'est secondaire [Vulgarisation, Paris]

... il s'efface devant l'idée que la reconnaissance mesurée à l'aune des droits d'auteur est bien trop maigre au regard du temps et du travail que représente un livre.

Un auteur n'estime jamais que sa rémunération soit suffisante ! [...] On vous apporte une rémunération pour un travail que vous avez fait... tout travail mérite salaire ! [Vulgarisation, Paris]

L'expression "droits d'auteur " entraîne, il faut le noter, une réponse sur le seul plan des droits patrimoniaux. Les droits moraux ne sont à peu près jamais abordés, sauf par un auteur de BD qui confond dans une même expression les premiers (limités dans le temps) et les seconds (imprescriptibles) $)^{48}$ :

48 Voir l'encadré juridique sur les droits d'auteur 
L'interviewer: Si je vous dis droit d'auteur, quelle sont les premières choses auxquelles vous pensez?

L'auteur : Je vous réponds entre $6 \%$ et $10 \%$ !

L'interviewer : et le droit d'auteur?

L'auteur: Ben le pognon! Le droit d'être payé pour son travail! Les royalties! Plus sérieusement, pour moi, il s'agit d'un droit imprescriptible sur mon œuvre, d'un droit à avoir une œuvre respectée, de choisir ce que j'en fais. Dans la pratique par exemple, là tel éditeur ne me rend pas mes droits sur une BD dont le tirage est épuisé. Donc elle ne peut plus se vendre, mais je ne peux pas en faire autre chose pour le moment. [BD, Sud]

Tout en estimant en général leurs droits trop faibles, les auteurs s'en sont pourtant rarement préoccupés. Quelquesuns ont eu une relation conflictuelle avec un éditeur pour lequel ils n'ont pas de mots assez virulents. Ils sont pourtant rares à exprimer des réserves sur le contrat d'édition et sur le montant de leur rémunération :

Ils ont multiplié les clauses pour diminuer à chaque fois la part de l'auteur. Peut-être qu'ils ont diminué la part d'autres acteurs du livre par ailleurs mais en tous cas ils diminuent la part de l'auteur. II y avait même une clause à la fin où ils disent que s'il en reste beaucoup on a le droit de les solder et si on les solde à moins de $30 \%$ ou $35 \%$, ils ne me donnent rien du tout. J'étais étonné de ça, ça m'étonne que ce soit légal. [Premier roman, Paris]

Certains soulignent la multiplicité d'acteurs qui interviennent dans la diffusion et la distribution du livre, acteurs qui doivent également être rétribués et dont ils n'estiment pas qu'ils perçoivent une rémunération démesurée.

L'éditeur n'est pas le plus souvent désigné comme le responsable de la situation, pas plus que ne le sont les libraires, ces derniers souvent très bien considérés. Un auteur incrimine les maillons intermédiaires de la chaîne du livre,

Je dirais que c'est quand même honteux qu'un auteur sur le prix d'un livre touche seulement $10 \%$. Mais ce n'est pas la faute des éditeurs ou des libraires. Je ne sais pas, je pense plu- 
tôt que ce sont les diffuseurs et les distributeurs qui s'en mettent plein les fouilles. [Romancier, Paris]

Aucun auteur n'avance d'explication en termes d'offre et de demande, alors que plusieurs d'entre eux, du fait de leur position d'éditeur, évoquent l'ampleur de l'offre de manuscrits, la très (trop) grande quantité de titres publiés et la faible rentabilité du secteur. Selon Jean-Pierre Ohl, auteur et libraire, 600 nouveaux romans faisaient la rentrée littéraire de 2004, ils sont plus de 730 en $2008^{49}$. Les auteurs sont conscients du pouvoir de l'éditeur, qui est en mesure de refuser la plupart des modifications au contrat que l'auteur pourrait demander.

Quelques-uns pourtant adoptent des attitudes très critiques, et particulièrement, dans notre échantillon, les auteurs de $\mathrm{BD}$ ou ceux qui se consacrent uniquement à cette activité et essayent d'en vivre.

Quand on lit le contrat, je les trouve très mesquins, c'est chercher tous les prétextes pour baisser le pourcentage de l'auteur sur chaque truc. C'est mon impression. J'ai vu des contrats d'il y a 10 ans, un contrat d'aujourd'hui c'est 10-12 pages, il y a un peu plus de 10 ans c'était 2-3 pages et pourtant ce n'était pas des anges non plus, ils étaient là pour gagner de l'argent. Ils ont multiplié les clauses pour diminuer à chaque fois la part de l'auteur. " [Premier roman, Paris]

Pour conclure, on peut donc souligner que, pour la plupart de nos auteurs, la faible information sur le montant des droits d'auteur va de pair avec un intérêt relativement limité pour le nombre d'exemplaires effectivement vendu, comme si les chiffres étaient peu significatifs. Les auteurs les mieux informés citent des fourchettes assez larges, le succès se mesurant davantage en termes de réception critique. II s'agit toutefois pour certains d'un point sensible dans leur relations avec les éditeurs, accusés par certains et pour certains, quand il s'agit de rendre des comptes sur le

49 Jean-Pierre Ohl, “L'édition face à la dictature des tuyaux", Le Journal de l'école de Paris du management, 2008/4, $\mathrm{n}^{\circ} 72$, p.15-22. Selon la revue Challenges, en date du 26 août, l'année 2011 afficherait la plus faible programmation depuis 2001 (575 ouvrages). 
nombre d'ouvrages vendus, de retard, d'omission, voire de mensonge qui justifierait les pratiques que certains auteurs qualifient de "flicage " et par lesquelles ils cherchent à vérifier les informations fournies par leurs éditeurs.

\section{Un rapport de force asymétrique}

L'ambiguïté de la relation auteurs - éditeurs provient du fait que s'ajoute à ce "partenariat " dans la création, une dimension commerciale. L'éditeur est la personne avec laquelle l'auteur signe le contrat d'édition et celui qui lui reverse les droits d'auteur, le détenteur principal des chiffres de diffusion, voire l'unique pour la plupart des auteurs. Certains d'entre eux expriment bien la difficulté qu'ils éprouvent à gérer cette dualité du rapport à l'éditeur, et ce d'autant plus que, à l'image du milieu français ${ }^{50}$, peu de ceux que nous avons interrogés ont un agent ou un avocat. Certains auteurs assument explicitement le rôle d'" agent de soi-même " :

Disons que j'ai une casquette 'auteur' quasi amicale et puis une casquette négo de contrat', là je mets le moins d'affect possible car je m'en méfie beaucoup. C'est comme si je me mettais en mode 'agent' quand on parle argent. [BD, Paris]

L'agent littéraire, une norme dans le monde de l'édition en langue anglaise, reste rare en France, et réservé à ceux qui ont une importante activité de publication. C'est le cas par exemple de cet auteur qui a publié plus de soixante-dix livres, dont certains à gros tirages (plus de cent mille exemplaires): il insiste pourtant sur la qualité de la relation amicale avec son agent plus que sur les ressources que celui-ci lui procure 51 , alors même qu'il souligne le fait que son agent-avocat-ami lui a apporté de "meilleurs contrats", des occasions de diffusion, une présence sur le web :

50 Dont les pratiques évoluent pourtant ; cf l'étude du MOTiF (En avoir ou pas. L'agent littéraire en France, réalités et perspectives, juin 2010) qui en repère entre 250 et 300.

51 Un discours défensif attesté également dans une des seules enquêtes sur les agents littéraires en France: Juliette Jost, L'agent littéraire en France, Réalités et perspectives, Étude MOTif, 2010 ; 117 p. 
Maintenant j'ai un avocat, un agent, qui est un ami, donc je suis dans un truc assez gros. II y a toujours eu beaucoup de gens qui se sont intéressés à mon travail, mais là ça prend des proportions importantes, ça devient compliqué donc j'ai déchargé toute la partie commerciale, voire stratégique à mon agent qui s'occupe de ça, et donc je ne parle plus de ça (rires), je ne parle plus d'argent. (...) Ce n'est pas un agent, c'est un ami. [Littérature enfantine, Paris]

Quelques-uns de nos interviewés ont fait appel, s'ils estimaient nécessaire d'avoir un regard extérieur, à un avocat ou à un juriste, plutôt qu'à un agent pour la négociation de leur contrat. II s'agissait pour eux de ne pas être lésés dans l'établissement et la négociation de celui-ci.

La marche de négociation est réduite pour un auteur. Pour mon premier roman, je suis allé voir un avocat, il m'a dit de faire modifier telle et telle clause. L'éditeur ne voulait plus signer. Maintenant j'ai peut-être un peu plus de poids vu mes ventes mais je ne sais pas si c'est vraiment vrai. [Médecin, roman policier, Paris]

Les auteurs, surtout les plus expérimentés, développent donc différentes tactiques pour retrouver un pouvoir de négociation. Ils tentent de pallier leur manque d'expertise en termes de négociation en faisant appel à un avocat, à un syndicat d'auteurs ou encore (plus rarement pour ceux que nous avons interviewés) en se renseignant auprès d'autres auteurs sur les forums en ligne. La sollicitation d'un avocat permet à certains d'évacuer de leur relation avec l'éditeur la problématique financière, pour ne conserver que la relation de travail.

Si j'ai une question sur des droits, sur une clause ou n'importe quoi, je pose une question sur Internet et j'en ai plein qui viennent me donner leur avis. Je vais regarder sur un forum et si je n'ai pas la réponse à ma question, je demande directement à mes contacts auteurs s'ils n'ont pas rencontré ce cas de figure. [BD, Bretagne]

J'ai pris un avocat, dès le début, la signature du premier contrat. II vérifie, me conseille, négocie mes contrats. Ça me permet d'être tranquille de ce côté-là, et puis ça dépassionne les relations entre l'auteur et l'éditeur. L'avocat permet de rationnaliser la partie business de la relation auteur - éditeur. [Auteur-blogueur, Paris] 
Certains regroupements d'auteurs par spécialité offrent, comme c'est le cas pour la charte des auteurs et illustrateurs jeunesse ou encore le groupement des auteurs de bande dessinée du syndicat national des auteurs et des compositeurs (SNAC), une expertise et un soutien aux auteurs :

Oui, en fait je me suis inscrite au SNAC. Quand j'ai une question ou une clause que je trouve bizarre ou un truc comme ça, je l'envoie, ils ont un juriste et on en discute. Ça permet de savoir un peu ce que l'on signe. [BD, Bretagne]

Les auteurs, les débutants en particulier, soulignent leur impression d'un rapport asymétrique dans la négociation financière avec l'éditeur, d'abord en raison de la difficulté pour un auteur de se faire publier, mais aussi souvent par manque d'informations sur les pratiques habituelles en la matière.

Mon pourcentage c'est entre 10 et $12 \%$. J'ai 20 exemplaires gratuits et tout le reste c'est un tiers du prix si j'en veux plus. Concernant le tirage entre 8000 et 10000 en fonction des ventes je pense, on n'en discute pas vraiment avec $X$, je pense [que mon éditeur] me ment, ce n'est pas un sujet de discussion, tout ce qui est commercial est opaque pour l'auteur. Aucun moyen de savoir les retours de stock, combien elle vend, etc... On n'a pas d'informations neutres, exogènes à l'éditeur. On est obligé de leur faire confiance, il n'y a pas de vérifications réelles. C'est comme une bouteille à la mer, on donne son bébé et on ne sait pas comment il va être géré, ni le discours interne etc. On n'a aucun accès à ces infos. [Médecin, Roman policier, Paris]

Cette insatisfaction est d'ailleurs relayée par certaines associations en charge des auteurs, comme en témoigne le baromètre des relations entre auteurs et éditeurs établi par la société civile des auteurs multimédias.

\section{j) Baromètre 2010 des relations Auteurs / Éditeurs établi par la SCAM}

- La reddition des comptes semble se dégrader. $46 \%$ des éditeurs respectent l'obligation légale de la reddition des comptes (49 \% en 2009), $18 \%$ ne la respectent jamais (16\% en 2009). 
- Lorsque la reddition des comptes est effectuée, elle n'est accompagnée d'un règlement des droits que dans $47 \%$ des cas (55 \% en 2009).

- Le délai d'envoi des relevés de comptes s'allonge. Dans $43 \%$ des cas, cet envoi a lieu entre trois et cinq mois après l'arrêté des comptes, alors qu'il ne représentait que 38 \% en 2009.

- La moitié des auteurs doit toujours écrire pour réclamer les comptes alors que la loi impose à l'éditeur de les adresser tous les ans.

- Pour la moitié des auteurs, les relevés de comptes transmis par les éditeurs ne sont ni clairs, ni complets.

Cette asymétrie est renforcée par l'opacité qui entoure, pour les auteurs, leurs chiffres de vente et le montant des droits à l'étranger, gérés par l'éditeur. Ces chiffres sont très souvent peu ou mal communiqués à l'auteur, de sorte que l'auteur ne peut faire valoir le succès de ses ventes pour mieux négocier son contrat sur ses livres suivants. Cette remarque doit bien sûr être nuancée dans la mesure où les auteurs ayant déjà connu un succès ont un poids plus important dans la négociation, et peuvent, éventuellement, avoir quelques exigences sur le montant de leur à-valoir et le pourcentage qu'ils touchent sur les ventes. Leur capacité à changer d'éditeur est également supérieure, ce qui peut permettre de rééquilibrer le rapport de force.

Face à cette différence de poids entre éditeur et auteurs, ces derniers opposent des attitudes variées. Les auteurs débutants se révèlent souvent moins exigeants sur la rémunération qu'ils perçoivent en tant qu'auteur, d'autant plus qu'ils occupent généralement, à côté de leur activité d'écrivain, une autre fonction professionnelle qui leur assure des revenus. Leur objectif est avant tout d'être publié, la rémunération apparaissant comme une question annexe.

Lorsqu'on publie un premier roman, on n'a à peu près aucun pouvoir de négociation, on est déjà content d'être publié. [Premier roman, Paris] 


\section{Les droits secondaires}

À côté de la perception de revenus directs, issus de la vente des livres (ou dans certains cas de l'adaptation audiovisuelle des œuvres), les œuvres écrites sont également susceptibles de bénéficier de droits " indirects " ou seconds, droits de représentation, de traduction, droits numériques, mais aussi rémunération aux titres de la copie, ou du droit de prêt en bibliothèque... Les premiers relèvent du contrat entre l'éditeur et l'auteur, les seconds sont le fruit d'une gestion collective et passent par des organismes de gestion des droits; les revenus concernés sont modestes (provenant essentiellement des droits liés au prêt et à la copie, mais reposent sur des filières de collecte et de redistribution d'une particulière complexité.

\section{k) La gestion collective des œuvres écrites}

Trois sociétés en avaient la charge au début des années 1930 la gestion collective des œuvres ; elles sont aujourd'hui 27 dont dix concernent l'écrit :

- Société des auteurs et compositeurs dramatiques (SACD), créée en 1777 ;

- Société des auteurs dans les arts graphiques et plastiques (ADAGP), née en 1953 ;

- Société de la propriété artistique et des dessins et modèles (SPADEM), créée en 1954 et mise en liquidation en 1996 ;

- Société civile des éditeurs de langue française (SCELF) qui date de 1960 ;

- Société civile des auteurs multimédia, (SCAM) née en 1981

- Centre français d'exploitation du droit de copie (CFC), créé en 1996, représente l'ensemble des ayants droit du livre et de la presse, pour le texte et l'image.

-Société française des auteurs de l'écrit (SOFIA) 1999, droit de prêt en bibliothèque, créée à l'initiative de la SGDL puis du SNE

-Société des auteurs de jeux (SAJE) née en 1997

-Société des éditeurs et auteurs de musique (SEAM, créée en 1998, au croisement des domaines de la musique et de l'écrit (droit de reprographie des partitions) 
-Société des auteurs de l'image fixe (SAIF), créée en 1999

Peuvent également concerner les auteurs de livre: la Société civile des auteurs, réalisateurs et producteurs (ARP), créée en 1987 ; la Société des arts visuels associés (AVA), formée en 2001 par l'ADAGP, la SAIF et la SCAM, vise la gestion d'œuvres constituées en tout ou partie d'œuvres des arts visuels; la SEAM pour les droits de reproduction de la musique imprimée, créée en 1988 qui rémunère les éditeurs ; la SAIF, Société des auteurs de l'image fixe, créée en 2005 ; la Société civile des éditeurs de langue française (SCELF) créée en 1959 qui joue un rôle d'intermédiaire entre des éditeurs d'œuvres littéraires et les sociétés d'auteurs, notamment la SACD, la SCAM et la SACEM.

La Commission permanente de contrôle des sociétés de perception et de répartition des droits de la Cour des comptes a la charge d'analyser et d'expertiser les activités des sociétés de gestion collective ${ }^{52}$.

Du coup et dans la mesure où ils représentent des sommes peu importantes, ces droits ne constituent pas un sujet majeur pour les auteurs, qui en ignorent souvent l'existence et pratiquement toujours le montant. Comme l'exprime notre romancier picard à une question sur les droits secondaires: "je n'en sais rien et vous ne devez pas avoir beaucoup d'auteurs qui savent ça!" [Romancier, Picardie]

Les droits d'adaptation occupent en revanche une place nettement plus importante aux yeux des auteurs interrogés. A minima, les auteurs savent qu'ils font l'objet d'une clause spécifique dans le contrat, qui stipule le partage des revenus (50/50, voir 70/30 pour certains). Dans un cas spécifique [Policier, Roman policier, Paris], un travail antérieur dans le secteur du cinéma a permis à l'auteur d'avoir

\footnotetext{
52 La complexité, l'opacité plutôt des relations juridiques et économiques entre ces organismes en charge de la gestion des droits, sont telles que la Cour des comptes a dû produire nombre d'organigrammes extrêmement détaillés pour en rendre compte. Comme le dit avec ironie le rapport 2010: "II n'est guère démontré que ces intermédiations multiples et de pure forme aient contribué à la transparence des relations économiques entre les sociétés partenaires." Voir en ligne son huitième rapport annuel de mai 2011.

www.ccomptes.fr/fr/CPCSPRD/Accueil.html
} 
l'idée de conserver ses droits: ainsi a-t-il pu refuser les adaptations qui ne lui convenaient pas et obtenir un traitement plus favorable pour celles qu'il a acceptées.

Les droits numériques constituent alors un entre-deux. Certains auteurs les considèrent comme un droit secondaire, traité par une clause spécifique (dont ils ne se souviennent en général pas) du contrat papier. Cette absence de conscience de l'enjeu du numérique, pourtant jugé comme essentiel par d'autres, est alimenté par l'absence d'information sur les adaptations en numérique, qui ne sont en général pas notifiées aux auteurs.

Certaines tensions sont maintenant liées à la façon d'envisager ce droit. Est-ce qu'on le lie au droit principal, au droit secondaire, est-ce qu'on l'inclut dans le contrat? Qu'en est-il des possibilités d'abonnement et de flux qui donneraient accès à un certain nombre d'œuvres? [Homme de lettres, Paris]

De manière assez claire, les droits numériques, comme pour certains les droits de traduction, même s'ils sont considérés comme importants, sont le plus souvent méconnus, à la fois parce que l'auteur admet n'y avoir prêté qu'une attention distraite, mais aussi faute d'informations en provenance de leur éditeur. Carence qui est soulignée par plusieurs de nos auteurs :

[Les droits pour le numérique sont] inscrits dans le contrat que je signe, mais après je ne sais même pas quel titre est vendu en numérique ou pas, ça ne m'appartient plus. Dans les contrats, il y a des alinéas et des alinéas, en droit d'auteur, votre image ne vous appartient plus ; après l'éditeur en fait ce qu'il en veut. Je ne sais même pas si je suis traduit à l'étranger. J'ai demandé plusieurs fois mais on ne m'a pas répondu. Je trouve ça dommage. [Nutrition, Paris]

II faut ajouter qu'en général pour nos auteurs les revenus provenant des droits secondaires sont faibles, voire nuls, ce qui contribue à leur indifférence. Comme le dit justement notre homme de lettres parisien "C'est un sujet sur lequel il y a beaucoup de discours et peu de revenus. ". Ou de manière plus virulente un bédéiste :

Pour moi, le numérique, c'est de la poudre aux yeux, c'est un non-sujet. Les éditeurs s'en mettent plein des poches et les au- 
teurs n'ont que les miettes de leur propre travail. (...) En fait, on veut nous occuper avec ce sujet et pas sur le droit au robinet! [BD, Sud]

Les auteurs ayant signé des contrats plus anciens sont parfois mieux au fait de ces questions, ayant dû signer un avenant spécifique pour le numérique. En tout état de cause, les auteurs informés semblent se sentir mieux fondés à négocier leur pourcentage sur les adaptations numériques, s'appuyant d'une part, sur des arguments de coûts inférieurs...

En revanche, je me suis accrochée avec mon éditeur sur I'histoire du numérique justement, parce que lors de mon dernier contrat, ou avant-dernier qui est le premier qui stipulait les histoires de vente numérique, à l'époque mon avocate m'a dit qu'elle avait des auteurs à $50 \%$, alors que moi ils me proposaient $8 \%$. Alors ça ne leur coûte rien, c'est une mise en ligne, il n' y a pas de papier, pas d'imprimeur, pas de déplacement, pas de libraire il y a rien, et mon avocate m'a dit "c'est absurde". Et mon éditeur m'a dit "c'est absolument hors de question" ; j'ai une amie dans une autre maison qui m'a dit “il faut comprendre, on est à l'aveuglette, les éditeurs dépensent des millions pour aménager leur plateforme" etc., et finalement la négociation s'est terminée à $9 \%$, ce que je trouve dérisoire, et je ne comprends toujours pas ce chiffre. [Romancière, Communication, Paris]

...et d'autre part, sur un prix plus faible des titres numériques, la hausse du pourcentage permettant d'assurer une rémunération constante par vente ${ }^{53}$.

Sur le papier j'ai le classique 10-12-14\% progressif et sur le numérique je m'attendais à avoir plus et ce n'est même pas progressif, même si je sais très bien que je n'atteindrais pas le nombre en papier pour être progressif, sur le principe ça m'étonne. Je pense que ça devrait être $20-25$ voire $30 \%$. Ils se plaignent en disant que c'est embryonnaire. Mais il y a beaucoup moins de frais même s'ils prétendent qu'il y a des frais de plateforme etc.; il y a beaucoup moins de frais d'impression et surtout de transport des livres - les invendus qui reviennent. Et

${ }^{53}$ Dans le cas du livre numérique, la notion d'exemplaire n'a pas vraiment de sens, aussi nous préférons ici traiter la question en termes de ventes. 
surtout c'est moi qui l'ai écrit quand même, j'ai fait tout le travail, ils n'ont rien fait, ils prennent déjà beaucoup par rapport au travail. S'ils l'avaient bien défendu, s'ils avaient fait quelque chose, à la limite je ne me plaindrais pas autant. [Premier roman, Paris]

À un extrême toutefois, un des auteurs interrogés est parvenu à obtenir une cession très courte ( 3 ans) des droits numériques, sur l'argumentaire d'un paysage très changeant et de la mise en place alors en discussion d'un prix unique du livre numérique, maintenant acté.

On a signé un contrat pour l'édition numérique, c'est un contrat de trois ans, parce que la loi sur le prix unique du livre va repasser dans cinq ans, donc j'ai dit à mon éditeur qu'on reverra après. [Vulgarisation, Paris]

On notera que l'émergence relativement récente de la question des droits numériques de l'auteur dans les contrats a renouvelé et intensifié le mécontentement des auteurs à propos de leur rémunération, qui devrait selon eux pouvoir être augmentée. En effet, ils estiment que le numérique permet des coûts de publication réduits pour l'éditeur. Le fait que les éditeurs leur proposent le même pourcentage pour leurs droits sur les livres papier et sur les livres numériques est interprété par certains comme le signe d'une injustice plus générale dans la rémunération des auteurs et une tentative de l'éditeur pour préserver ses marges, voire les accroître. Certains revendiquent que soit opéré un rééquilibrage du partage de la valeur sur le livre numérique, avec une diminution du prix de vente pour le lecteur et une augmentation du pourcentage perçu par l'auteur.

On fait sauter entre 60 et $70 \%$ du prix du livre en passant au numérique, la diffusion et la distribution disparaissent. Donc encore une fois tant mieux, mais il faut véritablement que ça se répercute sur les prix parce que dans ces cas-là nous on touchera plus que 8 ou 10 ou $12 \%$ et moi je préfère toucher $20 \%$ sur $5 €$ que $8 \%$ sur 15 , et encore une fois avoir plus de lecteurs ", [Romancière, Communication, Paris]

Au niveau des droits numériques, les éditeurs sont en train d'essayer de faire un sorte de nous octroyer le même pourcentage que pour les éditions livre sauf qu'il y a quand même l'imprimeur, le diffuseur et compagnie qui sautent, donc il n'y a 
pas vraiment de raisons pour que nos droits ne soient pas plus hauts. Ça c'est un peu le sujet d'achoppement ces temps-ci avec les éditeurs. [BD, Bretagne]

Aujourd'hui le problème est cette clause numérique. C'est un 'pain dans la gueule' ! On nous donne de moins en moins et nous demande de plus en plus. Et de fait le déséquilibre se renforce. L'éditeur est Majeur, l'auteur est Mineur. L'éditeur tient les rênes, comme dans une relation parent-enfant et pas du tout dans une prétendue relation de coopération! Or, la relation devrait être d'égal à égal; a fortiori parce que les lecteurs achètent des auteurs et non des éditeurs ! Ils font de l'argent sur notre dos. II faut rééquilibrer les relations, car dans l'état ça n'a rien d'un partenariat. [BD, Paris]

Le numérique a ainsi rendu pour eux palpable le manque de visibilité sur la répartition de la valeur au sein de la filière et concentré leur ressentiment. Certains rattachent toutefois la faiblesse des rémunérations proposées au moindre développement, à l'heure actuelle, du marché du livre numérique.

\section{Le prix du livre comme marqueur}

Pourtant, d'autres auteurs, partant de l'idée que les droits d'auteur constituent une reconnaissance du travail fourni, craignent que la baisse du prix liée au livre numérique ne dévalorise leur travail. L'un d'entre eux exprime clairement l'existence, à son sens, d'une hiérarchie de la valeur culturelle entre livres pratiques et romans. Les premiers, seraient plus faciles à écrire, souvent vendus au même prix que les seconds qui demanderaient davantage d'efforts.

Si évoluer ça veut dire mettre tous les livres au même tarif, alors non, et c'est ce qui se projette! Si vous mettez tous les livres à $5 €$, quand certains mettent des années à écrire un livre et d'autres un mois [...], ce n'est pas égalitaire. C'est bien de hiérarchiser les choses même au niveau des prix. [Vulgarisation, Paris]

Le prix est alors vu comme une marque de la valeur culturelle des ouvrages, un signe de qualité et une réduction du prix fait craindre une marchandisation de la littérature, à l'image de ce qui s'est passé dans le domaine de la musique. 
Il y a un effet "chiffre d'affaires " mais le pire effet est que si on analyse le comportement des consommateurs de musique, on s'aperçoit que la valeur de la musique a baissé. [...] Je ne serais pas étonné demain que la valeur d'un album soit de 3,50€. Du coup, je trouve qu'il faut éviter cela sur le livre, éviter cette dévalorisation. [Romancier Picardie]

De manière intéressante, un auteur oppose implicitement le prix du livre papier, en version poche, à celui du numérique, très proche, désignant le premier comme plus authentique que le second.

C'est disponible sur le site de l'éditeur et sur d'autres sites aussi, spécialisés un peu là-dedans, et ça coûte six euros, c'est un peu moins cher que le vrai prix. [Jeune auteur, Paris]

Les auteurs traitant de ce sujet s'accordent en effet à penser que le livre numérique a vocation à être moins cher que sa contrepartie physique, critiquant à cette occasion les politiques tarifaires des éditeurs, qui pratiquent des prix trop élevés par rapport à une cible évaluée, par l'un d'entre eux, aux alentours de $5 €$.

II y a plusieurs choses que je ne supporte pas [...]. [Les livres numériques] sont verrouillés par des DRMs. En plus ce n'est pas comme s'il y avait une contrepartie positive en termes de prix: la plupart coûtent aussi cher que l'édition papier existante. [Science-fiction, Angleterre]

Pour moi, le livre numérique doit coûter moins cher qu'un Poche, il doit couter $5 €$. Parce qu'à l'arrivée, on ne possède rien, on ne va pas le faire lire, enfin j'imagine que les gens peuvent le passer mais on sait qu'un livre fait au moins trois lecteurs, donc il y a une justification du prix ; là, une fois que c'est lu, c'est terminé on n'a pas le plaisir de l'objet [Romancière, Communication, Paris]

Toutefois, et comme dans l'univers physique, les décisions de prix, tout autant que de diffusion au format numérique, échappent très largement au contrôle de la plupart des auteurs, sauf pour les rares qui ont pris ex ante des positions affirmées sur ce point. 


\section{Le statut de l'auteur en question}

On le voit, les questions du contrat et de la rémunération de l'auteur, comme celle à un autre niveau du piratage, interrogent le statut de l'auteur: un professionnel qui se consacre à l'écriture à plein-temps ou un amateur qui tire ses revenus d'autres ressources ? Cela détermine en effet sa capacité à assumer le coût nécessaire pour proposer de nouveaux projets d'ouvrages, y compris les plus ambitieux et chronophages. L'auteur est-il un acteur économique parmi d'autres ou doit-il lui être accordé un statut privilégié du fait de l'investissement et du temps nécessaire à la production d'un ouvrage ? Au sein-même de notre échantillon, les positions divergent, notamment avec les différents statuts des auteurs. Ce sont surtout les auteurs qui vivent de leur plume qui se montrent les plus inquiets sur leur capacité à continuer à en vivre.

J'arrive à en vivre, ça irait mieux si mon éditeur ne coulait pas avec les droits d'auteur qu'il me doit. A part ça, ça va. Mais en édition, en ce moment, c'est vraiment la crise. Les éditeurs ne publient plus de nouveaux trucs, parce qu'apparemment les BD ne se vendent pas ces temps-ci. Là j'en suis réduite à faire de l'illustration ou des boulots de commande, des trucs comme ça. Heureusement que j'ai ça. Là, je ne fais qu'illustrer une méthode qui existe déjà en anglais. Je bosse un peu pour des magazines, pour un site Internet aussi, je fais des petites pages de BD... donc voilà, à droite à gauche. " [BD, Bretagne]

Beaucoup témoignent d'interrogations face aux mutations de la filière du livre (et plus largement de la culture) avec le développement du numérique; ces transformations qu'ils voient comme fondamentales interrogent le devenir du métier d'auteur, comme l'explique ce littérateur, un exemple parmi beaucoup d'autres :

On peut aussi se demander si on n'est pas en train d'assister à une mutation, et de changer de rapport de force dans le milieu de la culture et entre médias, et on peut se demander par-delà la question du livre si certains n'essaient pas de remettre en cause un certain nombre de principes du droit d'auteur qui permettaient jusque-là à certains auteurs d'être professionnels ou semi-professionnels. Là j'ai l'impression qu'on assiste à une mutation très profonde, qui va bien au-delà du droit numérique. Est-ce-que le papier parviendra à se maintenir? Quels seront 
les secteurs de la création qui seront absorbés par le numérique ? Quelle sera la place de la gratuité ? Comment protéger des auteurs sans tomber dans la répression ? Comment garder dans la création la part de générosité qui lui est inhérente? II s'agit pour les auteurs d'un travail. Ce sont des débats de fonds qui appelleraient des réflexions poussées que je ne trouve pas beaucoup pour le moment, ni du côté des défenseurs du droit d'auteur, ni du côté du copyleft et du partage, qui sont parfois un peu naïves et déconsidèrent la réalité du monde des auteurs qui se paupérise et auquel, si on n'y prend pas garde, des pans entiers de la création seront menacés. Ce sont des questions très complexes auxquelles on ne peut pas répondre en trois minutes, mais ce sont des débats autrement plus profonds que de savoir si on doit changer les contrats simplement des auteurs ou non pour le volet numérique dans l'édition. [Homme de lettres, Paris]

Un auteur de notre échantillon, débutant et ne vivant pas de sa plume, estime au contraire que le travail de l'auteur s'inscrit déjà dans une logique économique et qu'il doit trouver les moyens d'assurer sa subsistance et de mener en parallèle son activité d'auteur.

Pour moi le discours des maisons de disques est un discours très corporatiste, je ne sais pas si vous avez lu le rapport du Club des 13 de Pascale Ferran. C'était il y a quelques années, elle avait fait un discours larmoyant aux César et après elle avait écrit ce rapport pour dire ce qu'il fallait faire pour sauver la création cinématographique et tout ce qui s'en dégageait c'était qu'il fallait des subventions pour le cinéma pour que les gens du cinéma puissent vivre correctement... Moi j'admire plus Céline qui était médecin le jour, qui écrivait la nuit et qui a écrit des livres qui plaisent encore aujourd'hui. II y a un autre discours qui m'énerve aussi dans ces discours là c'est quand on parle de 'juste rémunération'. Avant le numérique il n'y avait pas de juste rémunération, après il n'y en aura pas non plus donc ce n'est pas ça la question. II y a rien de juste là-dedans, c'est juste les rapports de force de l'économie. [Premier roman, Paris]

L'asymétrie ressentie par les auteurs est forte, même semble-t-il lorsque la reconnaissance d'un écrivain, ses chiffres de vente, sa notoriété médiatique lui ouvrent les portes d'un grand nombre d'éditeurs. Comme si la transformation du livre en objet marchand mettait facilement 
l'auteur dans une position d'incertitude. Incertitude aggravée par ce qui est présentée par nos auteurs comme des pratiques très opaques des éditeurs pour tout ce qui relève de l'exploitation marchande des ouvrages. Ces pratiques ne sont pas plus claires lorsque l'on aborde la question de la numérisation des ouvrages.

\subsection{L'éditeur et le numérique}

II ressort des entretiens, en particulier de ceux avec les auteurs les plus impliqués dans les développements numériques, que les maisons d'édition se montrent encore assez frileuses en matière d'investissement dans le livre numérique. En effet, plusieurs auteurs, notamment ceux du secteur de la $\mathrm{BD}$, estiment que le marché du livre numérique reste encore marginal et surtout non rentable. II existerait ainsi une sorte de disproportion entre l'écho important consacré au livre numérique dans les médias et dans les divers débats sur le sujet, et la réalité du marché dans laquelle le livre papier demeure indispensable, étant donné les modèles économiques actuels.

Tiens par exemple, sur le site Comptoir de la BD, (géré par Le Monde),, on trouve toutes les semaines au moins 4 à 5 sujets qui traitent de la BD numérique, alors que tout le monde s'en fout car ça ne représente pas d'argent. Le seul sujet qui mérite d'être traité c'est le partage des revenus et les avances. Et les blogs, qui sont la chambre d'écho du net ne fonctionnent pas sur ces sujets. Le moindre péquin qui a un compte Twitter peut écrire et twitter n'importe quoi sur la BD, mais pour les vrais auteurs les droits numériques, c'est la petite caillasse, la petite monnaie. [BD, Paris]

Ce qui me frappe le plus dans le numérique, c'est que les ebooks, les liseuses, etc... forment une réalité qui occupe énormément de conversations mais qui reste économiquement dérisoire pour tous les auteurs et les éditeurs ; il y a donc une opposition entre les conflits que le numérique peut engendrer et leur réalité actuelle. Le numérique fait fantasmer pour l'avenir, et on sait aussi qu'il y a une place pour cela, on le voit déjà dans les marchés anglo-saxons mais pour l'instant c'est un sujet sur lequel il y a beaucoup de discours et très peu de revenus. [Homme de lettres, Paris] 
Les auteurs eux-mêmes se montrent peu impliqués et informés lorsqu'on évoque avec eux les possibilités du livre numérique, notamment quelques-uns des romanciers que nous avons rencontrés, attachés au papier et à la linéarité de la lecture, en raison d'une certaine conception de l'écriture et de la littérature.

S'il y a un endroit où la numérisation menace plutôt qu'ajouter quelque chose, c'est bien dans la lecture comme moment où on se coupe du monde et on suit le raisonnement. C'est très menacé ça par la génération zapping aux supports multiples et simultanés. [Jeune auteur, Paris]

D'autres, plus proches de la culture numérique, évoquent les problèmes de compatibilités des formats qui peuvent rendre rapidement obsolescent un ebook. Par ailleurs, le savoir-faire des maisons d'édition en matière de développement de livres numériques ou d'applications en ligne dérivées de l'ouvrage papier semble encore limité. Certains ont eu un ouvrage publié par leur éditeur en format numérique homothétique, disponible sur les principales plateformes de vente en ligne. Les initiatives les plus innovantes de type application dédiée sont toutefois plutôt le fait de l'auteur ou de professionnels rencontrés par ailleurs.

J'ai rencontré un mec comme celui qui m'a fait le site, c'est-àdire un mec jeune, ambitieux dans la création, qui me comprenait, on avait les mêmes mots. Et donc, je lui ai fait confiance. C'était un an de travail pour cette application, petit travail pour moi, pas un gros. [Littérature enfantine, Paris]

Pour un de nos auteurs, certains intervenants du marché cherchent à coller artificiellement des dispositifs pour immobiliser et figer les propriétés du marché du livre actuel. De son point de vue, cette dynamique est difficilement compréhensible d'autant plus que cela ne se reflète pas en des avantages économiques qu'on retrouverait ailleurs dans la chaine de valeur.

Les auteurs interviewés n'ont finalement qu'un intérêt relativement limité pour le livre numérique, jugé encore peu mûr ; son caractère émergent, les changements constants, les polémiques contribuent à nourrir les interrogations et les réserves des auteurs. Les questions de pluralité des formats 
disponibles, de leur incompatibilité, des supports, etc. sont peu abordées. On relève pourtant, en particulier parmi les auteurs issus du monde anglo-saxon, une opposition massive à l'usage des DRMs.

Selon moi, le système des DRMs est immoral parce qu'il représente une tentative de démolir notre héritage culturel commun pour une question de coût d'accès et il impose des limites aux usages qui vont bien au-delà de celles exigées par la loi sur le copyright. [...] En gros, les DRMs n'ont rien à voir avec la protection des contenus, il s'agit de protéger l'argent des majors ; pas du tout de vous protéger vous utilisateur, lecteur ou spectateur. Non ils se protègent les uns des autres. Et en plus, cela ne fonctionne même pas. Toutes les solutions en termes de DRMs ont à ce jour échoué : il n'y a pas une seule chanson, film ou logiciel infecté de ce type de logiciel qui ne se soit retrouvé en ligne d'une manière ou d'une autre dans la semaine qui a suivi sa sortie officielle, et même généralement bien avant sa sortie officielle. Donc d'un point de vue purement économique, en termes de protection des contenus, les DRMs sont un échec cuisant, et le resteront. C'est également nuisible pour l'utilisateur : il n'y a aucun système de DRMs qui bénéficie au consommateur. Au contraire, cela a un impact négatif pour lui : cela coûte plus cher à développer, il y a des coûts liés à la licence, cela rend le matériel informatique plus cher et complexe, et ça restreint les usages pourtant légitimes du consommateur. Donc, ça rend le contenu moins attractif, en limite les usages, et s'avère douteux d'un point de vue légal; alors, pourquoi l'industrie est-elle déterminée à empoisonner de DRM tout ce qui vous appartient? C'est simple, ils veulent une plus grosse part du gâteau, et ces systèmes de protection sont un moyen de l'obtenir en s'assurant que les usagers auront du mal à mettre en ligne les fichiers des grosses entreprises du secteur. [Science-fiction, Angleterre]

Nos auteurs ont en général rapidement évoqué cette question des DRMs, sans doute pour la plupart faute d'y avoir été confrontés directement, mais, comme le montrent les forums de discussion des auteurs et sites variés, l'opposition est assez bien partagée, en France comme aux États-Unis. Ils sont avant tout considérés comme inefficaces car n'offrant qu'une résistance faible aux pirates tandis que la plupart les conçoivent comme une contrainte onéreuse et injuste pour le lecteur-acheteur dans la mesure où cela ne 
permet pas le prêt à l'entourage, ce qui aboutit donc à dévaluer l'expérience de la lecture numérique.

\section{Une désintermédiation largement récusée}

Malgré les facilités offertes par le numérique en matière de publication en ligne, la très grande majorité des auteurs interrogés n'envisage pas de s'auto-publier sur Internet, y compris ceux qui possèdent les compétences informatiques et l'expérience nécessaire (les auteurs de BD notamment). On l'a vu, l'éditeur joue pour eux un rôle incontournable, à la fois comme soutien dans le travail d'écriture, label pour le lecteur (ce qui bénéficie en particulier aux auteurs les moins connus), et comme clef d'accès aux canaux de promotion (médias) et de distribution classiques (libraires) qui restent à l'heure actuelle ceux qui, selon nombre d'auteurs, ont le plus de poids sur le marché. Sa valeur ajoutée n'est donc pas réellement remise en question même si les auteurs considèrent qu'il ne remplit pas toujours le rôle qui devrait être le sien. L'éditeur reste pour l'heure un métier à part entière si la relation qu'il établit avec l'auteur est marquée du sceau de l'ambivalence.

Non [je ne m'auto-éditerais pas], j'aime l'idée du travail qu'on fait avec l'éditeur. [...] Aujourd'hui c'est l'écriture qui m'intéresse, et je trouve que le regard de l'éditeur est intéressant, il a un regard expérimenté ; nous au bout d'un moment on ne voit plus les choses, on est trop dans le texte. Je comprends qu'on s'auto-édite mais je préfère rester dans une logique de partenariat avec l'éditeur. [Romancier, Picardie]

Pour moi les éditeurs ont un avantage par rapport aux maisons de disques, c'est qu'ils reçoivent des milliers de manuscrits chaque année et ils doivent les sélectionner; donc ils font un tri pour le lecteur et ça prend beaucoup de temps. Pour la musique, les chanteurs peuvent se faire connaître sur MySpace alors qu'on ne va pas lire un livre en PDF d'un inconnu. C'est cette implication-là qui justifie les éditeurs de livres. [Premier roman, Paris]

C'est sûr qu'avec le numérique l'autoédition sera de plus en plus facile. Je veux dire que l'on sera nombreux à pouvoir 'mal s'autoéditer' aussi bien qu'on peut être mal édité par un professionnel aujourd'hui. (rires) [BD, Paris] 
Même un de nos auteurs qui a une véritable expérience en matière d'autoproduction, avec plusieurs livres en ligne à son actif établit une hiérarchie entre livre publié on line et off line, justement à cause de l'expertise qu'apporte l'éditeur :

Oui [je me suis déjà auto-produit]! Pendant les trois premières années. [...] Ça a couté beaucoup d'argent... à l'époque quelque chose comme 15000 francs, et ça ne rapporte pas. Par contre, c'est un très bon facteur de visibilité et d'apprentissage. On fait les salons.... Et dès 1998, j'ai fait ma promotion sur Internet, j'avais donc une grosse avance sur les autres. Mais ce n'est pas possible de s'autoproduire trop longtemps, il faut savoir s'arrêter et passer chez des éditeurs, sinon on s'enferme dans l'amateurisme qui s'auto-congratule, et a fortiori avec le numérique qui facilite la production par la baisse des coûts. [BD, Sud]

Quelques auteurs de l'échantillon ont contourné les maisons d'édition en utilisant le site Lulu.com pour publier euxmêmes leur ouvrage, ce site dont un éditeur, Léo Scheer dit qu'il joue le rôle de "agent pour la Star Ac de l'écriture "54. Leur démarche procède pourtant d'approches différentes. Dans un cas, l'auteur a décidé d'utiliser cette plateforme car il n'avait pas réussi à faire publier son livre par un éditeur, et n'a pas poursuivi avec ce système pour ses ouvrages suivants, ayant trouvé une maison d'édition. II s'agit donc d'un cas relativement classique d'auto-publication par défaut, après refus de l'éditeur. L'auteur met en avant la facilité d'utilisation et la liberté offerte par la plateforme Lulu.com, mais souligne également la difficulté à assurer par soimême la diffusion et la distribution du livre lorsqu'on est peu connu, ce qui limite de fait les perspectives de ventes.

Mon premier livre a été publié en 2001 chez X. C'était un roman. Ensuite, j'ai publié un roman sur le net parce qu'il ne trouvait pas d'éditeur. Je suis passée par un serveur, ce n'est pas qu'un serveur en fait. Ce n'est pas de l'autoédition. Tu ne donnes pas d'argent. C'est Lulu.com. Tu mets ton livre en ligne. C'est de l'édition sur Internet pour les gens qui n'arrivent pas à

54 In Juliette Jost, L'agent littéraire en France, Réalités et perspectives, Étude MOTif, 2010. 
se publier. Ou qui ne cherchent pas d'éditeur et qui veulent le faire pour la famille... Tu définis un prix et le livre n'est fabriqué que si les gens l'achètent. Cela ne génère aucun frais pour toi. J'en avais acheté un certain nombre. [...] Lulu.com m'a bien plu. C'est très facile d'utilisation, très simple. C'est évident à faire. Tu choisis ta police, tu choisis ta couverture. Tu choisis ton prix, avec un prix de base : ce qu'il coûte à fabriquer (environ $7-8$ euros... je ne sais plus trop) plus on rajoute librement les droits d'auteur (je gagnais 5 euros par bouquin, c'est énorme). [...] J'ai même eu un article. Dans le Corsica. Du coup, ça a généré des ventes et des signatures. J'ai eu quelques bénéfices. [...] Financièrement, c'est un bon plan dans le sens où tu gagnes plus d'argent... mais il y a le gros problème de la distribution. Pas de diffusion, pas de distribution. C'est toi qui dois faire tout par toi-même. Cela peut très bien fonctionner pour quelqu'un de très connu. Ça a marché pour Nabe parce qu'il est très connu. Moi, ce n'est pas concluant pour la distribution et la diffusion. Je n'ai pas vendu à des gens à Nice, Marseille ou Paris. Seulement en Corse. J'ai fini par le retirer de lulu.com [Littérature enfantine, Ajaccio]

Dans les autres situations, les publications via Lulu.com correspondaient à un choix volontaire. Elles s'inscrivaient dans des démarches de livres collaboratifs, évolutifs et gratuits, auxquels participaient dans un cas des auteurs identifiés (à la manière d'un ouvrage de recherche), et dans l'autre un public large (concrètement toute personne intéressée par le projet). Dans les deux cas, les auteurs ayant collaboré à l'ouvrage n'ont pas été rétribués. On le voit, Lulu.com offre, selon ces auteurs, plus de souplesse que les modes de publication traditionnels et convient donc davantage à ce type de projet à visée non commerciale. Ce type d'auto-publication devient ainsi une alternative aux éditeurs pour des projets plus expérimentaux.

Il nous a semblé que la procédure d'écriture et de publication qui s'adaptait le plus à une telle démarche était l'expérimentation qu'on est en train de mener, dont les traits principaux sont la démarche collaborative, la mobilisation des outils numériques dans la diffusion et les remaniements progressifs du livre, et le choix d'utiliser Lulu comme plateforme de vente et comme éditeur 'papier'. [...] Le développement de ce livre se fait à budget nul. Pour cette raison, il n'est pas question ni de le faire payer en version PDF, ni de rémunérer ses auteurs. Le coût de la version papier correspond aux coûts du 
matériel et de l'utilisation de la plateforme Lulu, on n'y a rien ajouté. [Directeur d'ouvrage collectif]

Enfin, on notera que les auteurs anglophones de l'échantillon proposent une partie ou la totalité de certains de leurs ouvrages gratuitement sur leur site personnel. L'un pour répondre à sa démarche d'écriture collaborative et évolutive, et l'autre pour faire découvrir ses textes aux internautes, en accord avec son éditeur, et avec l'espoir que les lecteurs auraient par la suite envie d'acheter le livre papier.

En fait, la première édition de A... était un e.book, avec le consentement de mes éditeurs; le livre était sous Creative Commons avec certaines restrictions comme "pas de modifications" ou "pas d'usage commercial". Il y avait une note sur mon site qui rappelait que tant moi que mes éditeurs nous espérions que les lecteurs apprécieraient le e.book et que cela les conduirait à acheter une version papier du livre. C'est le seul rappel qui est fait. Je ne suis pas pour les jagscreens ${ }^{55}$. [Science-fiction, Angleterre]

\section{Vers de nouveaux intermédiaires ?}

À l'heure actuelle, la suppression de l'éditeur comme mailIon qui assure la médiation entre l'auteur et son public semble ainsi loin d'être une réalité et n'est pas vraiment envisagée par les auteurs. Même les plus réceptifs à la culture numérique s'associent à des éditeurs et ne les contournent que pour des cas particuliers, comme celui du livre collectif évoqué plus haut. En revanche, certains auteurs imaginent avec inquiétude ou espoir, comme on le verra dans les deux citations contrastées suivantes, qu'avec le développement d'Internet, les éditeurs pourraient être remplacés par d'autres médiateurs: les opérateurs de contenus web, dont le poids économique est devenu majeur et devrait continuer d'augmenter. D'où une possible obsolescence des contenus traditionnels :

55 ces “agaciels", selon le joli terme proposé par les Québécois, qui rappellent de manière régulière à l'utilisateur qu'il emploie une copie non enregistrée et qui le poussent à régler le montant de la licence pour bénéficier de celui-ci, éventuellement après la période de test gratuit autorisé. 
On a le sentiment qu'il y a certains auteurs qui luttent contre les éditeurs et ne se rendent pas compte que les éditeurs sont potentiellement leurs alliés parce qu'ils restent du côté des contenus alors qu'un opérateur de vidéo à la demande se fiche complètement des contenus, ce qu'il veut c'est assurer un flux aussi divers et continu que possible. II y a une mutation qui est déjà faite, mais là je crois que c'est peine perdue. [Homme de lettres, Paris]

Le numérique devrait permettre d'évoquer une refonte complète des rapports entre auteurs et éditeurs... Actuellement les droits pour le numérique ne dépassent pas $10 \%$. D'où vient que ce pourcentage doit être le même qu'en papier ? Alors que la structure de coût n'a rien à voir, par exemple en ce qui concerne les manipulations logistiques, physiques. II existe des auteurs qui se sont autoédites chez Apple : ils touchent 70\%! Apple peut tout à fait devenir diffuseur. [BD, Paris]

Dès lors, la publication d'œuvres littéraires s'inscrirait dans une logique économique transformée et serait confiée à des opérateurs dont ce n'est pas le métier propre (vs l'éditeur). Les conséquences seraient selon certains auteurs néfastes pour la production littéraire qui par nature s'inscrit dans le long terme. Ainsi, une œuvre n'est parfois reconnue que des années après avoir été écrite. Ce ne serait pas le cas de tous les livres pratiques dont, le plus souvent, l'obsolescence est forte et rapide, adaptable à la volatilité numérique: on se dirigerait alors pour ce type d'ouvrages vers une évolution du monde de l'édition en fonction certes des contenus mais surtout des formats qui viendraient déterminer des contenus. Autrement dit, le mode de l'édition se structurerait en fonction du champ technologique qui ouvre de nouvelles formes de temporalité pour le livre. C'est la question de l'édition comme secteur à part entière qui est ici posée.

Dans une logique purement économique qui sera celle des grands groupes qui vont maîtriser les nouvelles technologies, ce temps-là, le temps de la maturation d'une œuvre, de l'écoute d'un auteur ou de l'accompagnement je pense, ne sera plus pris. Une nouvelle temporalité donc, et l'émergence d'un marché qui veut utiliser des produits et les rentabiliser très vite ; et une contradiction entre ce qui peut être l'invention, que ce soit en termes d'idées ou en terme de forme et les nécessités d'un marché qui veut identifier des produits et les 
rentabiliser très vite. Donc cela ne veut pas dire que la création va s'arrêter mais qu'une certaine forme de professionnalité des auteurs sera mise fortement en péril. [Homme de lettres, Paris]

Ceci interroge également le modèle économique de l'édition qui devient non seulement contraint par le technologique mais à qui est imposée une dynamique forte de retour sur investissement et de politique financière de développement technologique. La prise de risque devient un élément moteur du choix éditorial car elle se décline sur un mode financier qui exige un retour sur investissement rapide. L'obsolescence des anciens modèles d'édition va en effet de pair avec de nouveaux rapports à la temporalité de l'œuvre comme nous l'avons dit. Or, cette accélération de la temporalité se traduit aussi en termes économiques.

\section{Vers un déplacement du risque lié à la publication ?}

Or, on peut penser que la présence d'un auteur sur Internet tend à minimiser la prise de risque financière pour l'éditeur. En effet, la présence des auteurs sur le web, en amont de la publication, pourrait devenir profitable à l'éditeur. Lorsque l'éditeur signe un contrat avec un auteur repéré sur le web, il acquiert non seulement l'œuvre à venir de l'auteur, mais également sa notoriété existante sur le web, soit un "produit " déjà " testé ", avec éventuellement un public minimum déjà constitué. Cette démarche permet à l'éditeur d'une part de réduire les risques liés à l'investissement qu'il réalise en contractant avec un auteur, et d'autre part de réduire ses coûts de développement: le "produit " lui parvient déjà construit, éprouvé et positionné sur le marché du livre pour un genre et un public. II devient également plus aisé pour lui et moins risqué d'investir de nouveaux segments éditoriaux.

Pour l'éditeur, l'auto-publication et l'auto-promotion des auteurs sur le web jouerait donc le rôle joué par les "pilotes" dans le secteur audiovisuel. 
Selon Menger ${ }^{56}$, l'artiste ou l'auteur est à la pointe du libéralisme : ce statut ne coûte rien à personne, 'l'artiste est libre' ! Mais ça en fait un maillon extrêmement faible de la chaîne. Tu vois, c'est très commode cette horde de jeunes auteurs qui ne demandent que le statut et la reconnaissance, sans vraiment voir les aspects financiers ! [...] Pour moi il y a un retournement quasi philosophique. On va nous dire "pourquoi vous faire une avance alors que l'album est déjà conçu et en ligne ? " ou encore pire "habituez-vous à avoir un deuxième métier"! Normalement ces auteurs doivent pouvoir gagner leur vie avec le boulot qu'ils font. Aujourd'hui, un jeune peut bosser sur un blog pendant un an, avec un boulot à côté, il va créer 46 pages qu'on va lui payer deux ou trois mille euros ! [BD, Paris]

Dans le cas de la bande dessinée, on voit des auteurs qui mobilisent les outils du numérique à leur disposition pour leur autoproduction (mise en ligne de leur travail, par morceau ou intégralement) grâce à des coûts de diffusion faibles et à leur autopromotion, se constituent en plateforme au sens économique du terme. En effet, ils deviennent un lieu d'échange et de transaction. Dans cette configuration, un auteur met en ligne une création qui attire un public, même minime. Ce public, si petit soit-il, représente une valeur. Une valeur sinon marchande, au moins de négociation, et de visibilité. L'auteur offre alors un double-produit : une $œ u v r e$ et un public. À la différence d'un média traditionnel, qui dans cette logique de plateforme vendrait d'une part son contenu à son public et d'autre part son public à des annonceurs, nous serions, dans le cas des auteurs, face à une forme originale de plateforme dans laquelle l'œuvre et le public sont simultanément "vendus" à un éditeur potentiel, ou à tout le moins valorisés auprès de lui. L'éditeur quant à lui acquiert par cette transaction, la création de l'auteur et de son public. Ce public ne se transformera pas automatiquement en clients de la maison d'édition, mais il constitue une base de "prospects" pour cet auteur et les autres auteurs de la maison d'édition. Ce sont autant de coûts de

56 L'auteur interviewé fait allusion au sociologue français Pierre-Michel Menger, spécialiste de l'art et des créateurs, auteur en particulier de Portrait de l'artiste en travailleur. Métamorphoses du capitalisme, Paris, La République des Idées / Seuil, 2003. 
promotion (marketing, buzz, service presse et critiques...) qui peuvent ainsi être, sinon évités, au moins réduits. Par ce biais, l'éditeur s'assure une association avec un auteur qui sous-traite déjà et sous-traitera sûrement gratuitement cette partie de la promotion des livres et de l'auteur; il assure l'animation de sa communauté de fans, gère lui-même une part des contacts presse, des participations aux salons, organise éventuellement des formes de teasing, des animations, des débats, au moment des sorties de ses livres...

Cette forme d'organisation en plateforme est particulièrement prégnante parmi les auteurs de bande dessinée. En effet, nous constatons parmi les auteurs interviewés, mais aussi ceux dont on a rencontré l'offre sur I'Internet, que ceux-ci mettent à disposition gratuitement des planches de leur BD. S'agit-il pour autant de gratuité ? II s'agirait bien plutôt d'une économie du don. Le don a lieu de la part de l'auteur: il donne une part de sa force de travail en mettant sa création à disposition des lecteurs sur l'Internet. Mais en échange, ces lecteurs, par leur seule fréquentation du site de l'auteur, sur lequel ils sont a minima comptabilisés, effectuent un contre-don en se constituant en une audience que l'auteur pourra valoriser, sous une forme monétaire (montant des avaloirs, pourcentage sur les ventes, etc.) ou sous une forme plus stratégique (liberté supplémentaire de proposition, souplesse des contrats, choix de prestataires ou d'artistes associés...) au moment de la signature d'un contrat avec un éditeur.

Ce contre-don peut visiblement prendre plusieurs formes en fonction des modalités de présence des auteurs sur Internet et des modèles choisis par eux. Ainsi l'on pourra trouver des contre-dons sous la forme de levée de fonds, de publicité, de réputation. L'auteur devient alors une plateforme traditionnelle, un média :

J'ai mis un message sur mon site web disant que j'espérais que les gens auraient envie d'acheter un exemplaire papier du livre, ou de faire un don pour m'aider à continuer ce travail. Je dis aussi explicitement que si les gens veulent être enregistrés en tant que "fan " de M... sur le site, il leur suffit de me le faire savoir. [Sciences sociales, États-Unis] 
II est intéressant de le relever dans nos interrogations sur les relations entre auteurs et éditeurs à l'ère du numérique, en ce sens qu'il y a constitution d'un public avant l'acte d'achat, contrairement à une démarche commerciale classique, y compris dans les industries culturelles. La revente ou la valorisation de ce public (ou notoriété) à un éditeur permet le passage de la création de l'auteur du web au papier. Ce faisant, il y a de manière concomitante passage du "gratuit" à un modèle payant pour le lecteur ; le papier ne pouvant être gratuit (dans ces cas de produits culturels non financés par la publicité). Ainsi pour le lecteur comme pour l'auteur le passage du modèle de don/contre-don à un modèle commercial classique faisant entrer la création dans une chaine de valeur et de redistribution monétaire, s'effectue en dehors de la plateforme initiale ; c'est-à-dire en intégrant une intermédiation nouvelle entre les deux acteurs que sont l'auteur et le lecteur, celle de l'éditeur (qui draine lui par sa position dans la chaine de valeur, les autres acteurs de la commercialisation du produit) : on semble dire ici que l'intermédiation de l'éditeur est nouvelle, or ce n'est pas le cas. C'est plutôt la relation au lecteur qui est ici innovante.

\section{Conclusion : le numérique déplace l'asymétrie, mais la rééquilibre-t-il ?}

Une des dérives potentielles qui a émergé à plusieurs reprises lors de nos rencontres avec les auteurs les plus avancés dans leur maitrise des outils du web, est la crainte que les éditeurs ne se déchargent de leur responsabilité dans le succès de l'œuvre, en externalisant de fait sur l'auteur les fonctions de promotion de sa création par les outils numériques. Ceci entraînerait certes une réduction des coûts pour l'éditeur, mais un accroissement de la tâche pour l'auteur. La crainte de la désintermédiation ne touche donc pas seulement les intermédiaires (qui sont ici les éditeurs, les libraires, les diffuseurs, etc.) mais aussi les auteurs. Cela semble relativement le cas pour les auteurs les plus présents a priori en ligne et pour qui l'utilisation de 
ces outils de visibilité et de promotion est " naturelle " voire " originelle".

Pour moi, les blogueurs sont de deux catégories : il y a les blogueurs de pures œuvres numériques, ils sont généralement déjà des auteurs et c'est un choix de faire des œuvres pour le web. Mais il y a surtout les "blogueurs aspirants auteurs papier ", qui espèrent être repérés et être édités. Eux font actuellement les frais du système numérique. Je m'explique, il y a une forme de délégation du risque éditorial de l'éditeur sur l'auteur: le blogueur prend sur lui de faire ses planches, dans son coin. Sa popularité est connue sur les sites. Et au final ils sont rachetés pour une bouchée de pain, par exemple entre mille et trois mille euros pour les droits d'un album, contre vingt mille euros pour une avance digne de ce nom (pour deux personnes: scénariste et dessinateur, pour un an de travail). Or c'est le rôle de l'éditeur de prendre ce risque. [BD, Paris]

Cependant, revers de cette crainte, on a vu par quelques exemples ici que le numérique permet aussi à l'auteur de reconquérir une part de maitrise dans la promotion de ses créations et sa notoriété, et ainsi de rééquilibrer le rapport de force existant avec les éditeurs. Notre auteur américain de sciences sociales, par exemple, a pu choisir, avec la maison Lulu, d'imposer la publication de son livre sous une licence Creative Commons :

Lulu est une entreprise qui comprend les changements de la communication. C'est un service d'auto-publication, une opération qui permet de faire de mon travail un livre qui peut être vendu par moi et par toute autre personne qui souhaite le distribuer. A l'automne dernier, quand j'ai commencé à travailler sérieusement sur le livre lié au projet, j'étais sous contrat avec l'éditeur qui a publié $W . .$. il y a quelques années. On a décidé de mettre fin à notre collaboration en janvier et mon agent littéraire a commencé à chercher un nouvel éditeur. Mon ancien éditeur n'avait pas de problème avec les Creative Commons, nous avons d'ailleurs publié le premier livre sous cette licence. Mais comme me l'a dit David, mon agent, lorsqu'on commençait notre recherche d'un nouvel éditeur, les possibilités risquaient d'être limitées parce que j'avais un unique critère non négociable: que le livre soit publié sous licence Creative Commons. [Sciences-humaines, États-Unis]

Lulu.com rend possible, comme d'autres éditeurs, dont le nôtre ici, les Éditions des archives contemporaines, la 
coexistence simultanée du livre papier et de sa version électronique, vus comme complémentaires et non concurrents. Mais, comme le montre la dernière citation, c'est au prix d'un travail d'intermédiation, ici rempli par l'agent, que l'auteur peut mettre en place une telle configuration. Pour la diffusion aussi, le face-à-face direct entre auteur et lecteur reste illusoire (ou accidentelle), la rencontre exige un lourd travail d'ajustement, qui doit être réparti entre l'auteur, l'éditeur, le distributeur... et les nouveaux intermédiaires, agents ou autres, qui, sans nul doute, en feront leur domaine d'intervention. 


\section{Conclusion générale}

En conclusion de cette enquête auprès des auteurs de livre, deux questions méritent d'être posées: d'une part, dans quelle mesure et sous quelles conditions peut-on dire que le numérique constitue un nouvel espace de création pour l'auteur de livre; d'autre part, en quoi le numérique interroge-t-il la propriété intellectuelle et comment penser leurs adaptations réciproques.

\section{Le numérique, vers un nouvel espace de création pour l'auteur?}

Le livre est un véritable vecteur d'innovation. Loin d'être un objet figé, il est au contraire d'une grande plasticité, ouvert et nomade, adaptable et flexible. Ouvert parce qu'il permet de nombreuses adaptations et des formats très divers; nomade parce qu'il peut être utilisé en tous lieux (sous la seule réserve qu'il y ait de la lumière !) ; flexible parce qu'il autorise de multiples usages, de la lecture cursive à la lecture savante, du loisir de l'enfant à la mémoire du savant... C'est ainsi qu'il a su accompagner les révolutions de la lecture, de l'édition, du savoir, comme l'ont montré les nombreuses transformations de la matérialité de son support, les évolutions de ses technologies d'impression, de son modèle économique, de ses rapports aux lecteurs, de son statut juridique, etc.

Pourtant, n'est-il pas menacé ? On nous dit que le nombre de gros lecteurs diminue inexorablement (Donnat, 2009), les gros lecteurs étant définis par les enquêtes publiques sur les pratiques culturelles en fonction du nombre de livres lus chaque année. Est-ce à dire que la forme est morte et appelée à disparaître ? Certes non : comme Roger Chartier (2001), nous pensons plutôt que continueront à coexister plusieurs formes de textualité : l'imprimé, le manuscrit et 
l'électronique; que la plasticité du livre lui permettra d'affronter le défi du numérique et que son avenir est devant lui. La définition du gros lecteur devra sans doute évoluer: le livre ne pourra plus seul prétendre qualifier le "lecteur", mais il ne sera pas non plus exclu de cette pratique. À l'issue de cette enquête, plus encore que dans ces prémisses, nous croyons en l'avenir du livre parce qu'il représente une clôture (la possibilité de donner une fin, d'inscrire le texte dans des limites), une sélection adressée (les limites d'un choix réalisé pour un lecteur, fut-il entièrement projeté), un regard personnalisé, une autonomie par rapport aux autres contenus, toutes caractéristiques que le texte numérique, dans sa prolifération et sa multiplicité, ne peut offrir.

Cependant, le numérique d'ores et déjà agit sur la production des auteurs. On a vu dans notre enquête la grande place qu'il occupe dans l'activité du plus grand nombre d'auteurs, dans la manière de construire les savoirs, de rédiger les textes, de produire du sens, de le comprendre et de se l'approprier. Les producteurs de connaissance ne sont pas les seuls touchés, mais aussi, comme on le voit à travers les entretiens, tous les auteurs, romanciers, dramaturges, auteurs de bandes dessinées... bref tous ceux, ou presque, qui ont pour ambition de produire des livres.

Les technologies numériques interviennent à toutes les étapes du travail de l'auteur : comme l'écriture, suivant Jack Goody (1979), le numérique sera un outil de transformation des connaissances et des sociétés humaines, ne serait-ce que parce que la mise en forme des données et les instruments de présentation des connaissances structurent la pensée. Le recours presque généralisé aux logiciels de traitement de texte (il faudrait même pour la plupart des auteurs dire Word tant l'influence de l'outil de Microsoft est massif), le stockage d'informations permis par l'ordinateur et ses capacités démultipliées, voire délégué à des supports externes, la mémoire des versions successives du texte dans leurs moindres détails, le décompte du nombre de signes, l'automatisation des corrections, les mises en page, polices et styles proposés, etc., agissent sur la manière d'écrire, sur la production de textes, comme sur leur lecture. 
Tout comme le livre, l'écriture n'est pas un élément figé mais elle se conçoit comme une économie de la trace et de l'effacement, une production non seulement de l'esprit de l'auteur, mais des interactions que celui-ci entretient avec son environnement numérique: encyclopédies et dictionnaires en ligne, témoignages, disponibilité des images et atlas sont autant d'éléments dont la facilité d'accès interagit directement avec l'élaboration du livre et le travail de l'auteur.

Le livre numérique, qui n'en est encore qu'à ses prémices, n'est que la forme la plus visible de ces transformations. Mais lui aussi permet de nombreuses déclinaisons: du livre dit homothétique qui mime le livre papier (sans arriver toutefois entièrement à égaler aujourd'hui, en l'état des dispositifs numériques de lecture, ses incomparables qualités) à un artefact-livre, ce modèle évolutif dont certains auteurs ont fait le choix de s'emparer. Dans les versions les plus avancées, où s'hybrident le texte, le son, l'image, le dialogue avec d'autres, la définition même de l'auteur se brouille, tout comme il devient parfois difficile de dire pourquoi on continue à nommer livre ce continuum de sons, de textes et d'images. Et pourtant, même avec des corrections successives, une démultiplication des versions remaniées, un travail collaboratif qui distribue l'autorité de l'auteur, avec le mixage de sources multiples, la notion de livre garde sa pertinence, quand elle permet de faire œuvre, de clore un texte, de marquer une étape.

II faut d'ailleurs noter qu'une telle évolution ne concerne aujourd'hui qu'un nombre limité d'auteurs (comme demain, sans nul doute, elle ne les touchera pas tous). Les auteurs réticents face à ce qu'ils considèrent être une intermédiation technologique perturbante, ceux qui privilégient un mode d'écriture et de publication traditionnels ont aussi leur place dans le monde du livre. En dressant une typologie de portraits d'auteurs au temps du numérique, notre enquête a d'ailleurs montré à la fois la place grandissante du numérique dans l'écriture (au moins comme source d'information et moyen d'échange) et la souplesse des modèles d'écriture. Elle n'a pas décelé d'opposition frontale entre l'auteur geek et l'auteur traditionnel, mais plutôt des modalités de fré- 
quentation différente du numérique et du Web, naviguant entre l'auteur affiché, peu familier des espaces numériques dont le rôle relève peu d'une activité numérique et l'auteur réseau, qui bénéficie d'une forte conscience numérique, entre l'auteur expérimentateur pour lequel Internet apparaît comme un support radicalement nouveau, un lieu d'expérimentation pour des pratiques d'écritures collectives et l'auteur commentateur qui découvre sur la Toile d'autres moyens de se rendre présent dans l'espace public.

Côté diffusion, de nombreux auteurs appréhendent le numérique comme un incontournable "système de communication " propre à établir des médiations inédites, perturbantes pour certains, productives pour d'autres entre auteur et lecteur. Dans cette reconfiguration, on l'a vu, la relation auteur-éditeur se transforme; l'auteur devient (ou peut devenir) progressivement un acteur central de la vie médiatique de son livre et de sa visibilité ultérieure. L'auteur n'est peut-être plus le seul dépositaire du livre, de son sens (s'il l'a jamais été) mais là le lecteur fait entendre son avis directement: le livre attire des contributions extérieures, il est facteur de conversations et d'échanges qui viennent alimenter sa popularité et sa visibilité, en même temps que celles de ses auteurs. Se créent donc une multitude de conversations autour du livre et sur lui, auxquelles l'auteur peut choisir de participer ou dont il peut préférer demeurer observateur, voire qu'il peut ignorer. Dans tous les cas, il lui appartient, en ces temps numériques, de décider, s'il souhaite faire de l'Internet une nouvelle tribune pour parler de son livre, le défendre, ou peut-être le continuer, le transformer, s'il accepte de répondre aux questions de ses lecteurs ou s'il préfère s'en remettre à d'autres. Ou encore s'il veut en faire le support de pratiques renouvelées d'écriture, parmi lesquelles le livre n'est plus qu'une fin parmi d'autres.

\section{L'auteur en sa propriété}

La notion d'œuvres n'est pas interrogée seulement par les livres "augmentés " ou "enrichis ", par l'usage d'Internet et par la production collective; le piratage, la circulation non contrôlée des textes questionne aussi leur définition. Que 
faut-il laisser en libre accès et jusqu'où aller dans les permissions? Comment tenir la balance équilibrée entre recherche de notoriété et protection de l'œuvre? Quelle doit-être la taille d'un extrait significatif dont on autorise la mise en ligne? Jusqu'où le piratage doit-il être accepté comme rançon du succès? Quels usages des textes peuvent être autorisés?

Ces questions illustrent la capacité du numérique à influencer la notion même de propriété de l'œuvre écrite. Tous les auteurs de notre enquête, des BDistes aux romanciers en passant par les auteurs de livres pratiques, considèrent le droit d'auteur in fine comme le seul élément tangible de leur reconnaissance. Ce droit ne se résume pas à la perception d'une rémunération, très faible au demeurant pour la grande majorité d'entre eux, mais il est à la fois un symbole d'appartenance à une profession (ou au moins à une pratique) et la reconnaissance d'un travail réalisé. Certains auteurs sont sensibles au risque de désappropriation de l'œuvre, rendu plus fort par les facilités des outils numériques: si le plagiat n'est pas nouveau, le numérique, avec ses outils de recherche et de copier/coller, son infinie disponibilité de contenus et ses capacités de stockage massives, rend beaucoup plus facile les "emprunts", les réutilisations, ou les recyclages des travaux d'autrui. Cherchent à y répondre un foisonnement de sites et blogs destinés à pallier ces risques de pillage (que certains auteurs diraient de flatteuses reprises) des œuvres écrites par des logiciels de recherche sémantique, voire par des techniques plus sophistiquées. L'Internet, qui permet la visibilité et la diffusion de l'œuvre écrite en même temps que la dissolution possible de la notion d'auteur, génère donc aussi les outils qui cherchent à prévenir cette dissolution et à protéger la propriété intellectuelle.

Plus brutale que ces emprunts, la diffusion non autorisée de textes intégralement copiés commence à se répandre, même si on est encore loin de la situation de la musique ou du cinéma. Le téléchargement illégal de livre toujours mar- 
ginal se diffuse à mesure que le nombre de versions numériques des livres augmente 57 .

Plusieurs remarques doivent d'abord amener à interroger la signification du piratage dans l'économie culturelle; si par principe pour ne pas dire par définition, le piratage (entendu comme prise de possession d'un contenu marchand) ne peut être que blâmé d'un point de vue éthique, notons pourtant que son impact sur les ventes est controversé. Nombre d'études soulignent la complexité des effets de ce phénomène au sein des industries de contenus. Par exemple, les plus gros pirates, ceux qui téléchargent le plus de contenus non autorisés, seraient aussi ceux qui en achètent le plus. D'autres études 58 s'emploient à montrer que le piratage peut avoir des effets économiques positifs : il peut être une manœuvre stratégique lorsqu'il permet d'opter pour discriminer les prix ou qu'il permet d'utiliser la croissance de la taille du réseau qu'il provoque pour bénéficier d'externalités additionnelles et atteindre ainsi plus rapidement une masse critique suffisante. Certains travaux notent également que le téléchargement illégal ne conduit pas systématiquement à une vente perdue (combien d'œuvres téléchargées pour n'en rien faire, pour les tester seulement, ou parce que le lecteur ne sait plus où il les a rangées...). Au-delà de ces assertions, plusieurs questions méritent d'être posées: l'usager a-t-il les moyens de vérifier le caractère licite de ce qu'il consomme? Doit-on penser (encadrer) la circulation des œuvres de la même façon lorsque l'échange se réalise dans la sphère marchande ou lorsque celui-ci est le fruit d'un partage non marchand entre usagers ? Comment dé-

57 Voir les études EbookZ du MOTiF, la deuxième datant de mars 2011 montre qu'il s'agit essentiellement de livres très grand public, que le piratage, tout en restant limité à une faible proportion des parutions principalement des bestsellers, s'accélère mais n'aboutit qu'à un faible nombre de copies.

58 Voir en particulier les travaux de Conner and Rumelt (1991), Takeyama (1994), Slive and Berhardt (1998), Shy and Thisse (1999), Han and Croson (2000), Bekir et ali (2010). Voir aussi "L'impact du piratage sur l'achat et le téléchargement légal. Une comparaison de quatre filières culturelles ", Irène Bastard -Télécom ParisTech, séminaire M@rsoin, les 26 et 27 mai 2011; ou encore l'article posté sur le site de la BBC en 2009. http://news.bbc.co.uk/2/hi/technology/8337887.stm 
termine-t-on le statut d'un contenu et les droits que son créateur lui attache?

Ces interrogations posées, comment sortir de l'opposition stérile entre des créateurs dont le seul rapport au numérique serait celui de victimes et un public qui n'aurait pour ambition que de consommer gratuitement les biens culturels?

Notre enquête montre clairement qu'il faut, pour sortir de ce vain antagonisme, repartir de la complexité des populations, des créateurs, des intermédiaires et des publics, tout comme il faut distinguer entre contenus, entre supports, entre auteurs; et ne pas amalgamer tous les publics, tous les usages. Côté créateurs, la très intéressante étude sur les musiciens conduite par Bacache-Beauvallet, Bourreau et Moreau (2011) a mis en évidence l'extrême diversité des créateurs, leur réaction fort différente face au numérique et leurs diverses trajectoires. Nous faisons l'hypothèse que la diversité est au moins aussi forte chez les auteurs de livre ; pour moins étudiée qu'elle soit, leur capacité à se saisir des possibilités du numérique pour la production et la diffusion de leurs œuvres est potentiellement importante, leur capacité à engendrer de nouvelles sources de revenus n'est pas inexistante. Côté intermédiaires, les professions vont certes être amenées à se transformer, mais les métiers en charge d'organiser la rencontre entre le lecteur et l'auteur restent indispensables, tant est complexe leur adéquation. Comme l'explique Bruno Latour ${ }^{59}$, la numérisation n'a pas seulement ajouté de nouveaux métiers, elle a rendu plus présents ceux qui existaient déjà : "plus on numérise, plus on matérialise l'ensemble des flux et des fonctions qui entouraient jusqu'ici le travail du livre sans qu'on s'en rende toujours compte.". Côté publics, enfin, rien ne vient conforter la thèse d'une dépréciation du livre60 : à entendre les auteurs

59 Latour, Bruno, "Plus elles se répandent, plus les bibliothèques deviennent centrales " BBF, 2011, 56-1, pp 34-36.

60 comme le soutient par exemple dans Le Monde du 3 février 2012 Yves Charles Zarka. Contre, par exemple Alain Kyrou qui note "L'objet livre donne par tout son art un sentiment de richesse qu'a perdu par exemple le disque, à cause du CD et de sa pauvre boîte en plastique, au contraire du vinyle dont on 
que nous avons interviewés et à voir la progression constante du nombre de nouveautés éditées depuis trente ans, la publication d'un livre reste l'horizon indépassable de nombre d'auteurs.

Mais le panorama n'est pas complet si on n'y ajoute pas la puissance publique, dont le rôle en matière de livres est particulièrement important en France. La loi dite Lang sur le prix unique du livre a eu certes des effets ambigus (ou du moins controversés ${ }^{61}$ ) sur le coût des ouvrages, la qualité du réseau des librairies, sur la diversité de l'offre...) mais elle semble reconnue comme un instrument déterminant de régulation de la librairie 62 . II lui reste à inventer pour le livre un nouvel instrument d'intervention qui à la fois préserve ses ambitions traditionnelles (favoriser la diversité culturelle et la diffusion des ouvrages) tout en s'adaptant aux nouvelles formes de diffusion et aux rapports transformés entre les créateurs et le public. Victor Hugo à la veille de la Troisième République en situait déjà l'enjeu 63 : "Le livre, comme livre, appartient à l'auteur, mais comme pensée, il appartient - le mot n'est pas trop vaste - au genre humain. Toutes les intelligences y ont droit. Si l'un des deux droits, le droit de l'écrivain et le droit de l'esprit humain, devait être sacrifié, ce serait certes, le droit de l'écrivain, car l'intérêt public est notre préoccupation unique, et tous, je le déclare, doivent passer avant nous. " Et il ajoute "Ce sacrifice n'est pas nécessaire " car il vient d'appeler les gens de lettres à définir les règles et lois de la propriété littéraire, en accord avec les droits du public.

Le droit d'auteur est l'élément symbolique mais aussi tangible de la rémunération de cette création. Scindé en droit moral et patrimonial, il défend à la fois le respect et la paternité d'une

avait annoncé à tort la disparition totale il y a vingt-cinq ans. " (in " Google et le syndrome Hadopi ", Multitudes, 40, 1, 2010)

61 cf Perona et Pouyet, 2010 ; Benhamou, 2003

62 Les aides directes étant selon Perona et Pouyet (2010) de faibles montants (en diminution de surcroît) et peu cohérentes.

63 Lors du discours d'ouverture du Congrès littéraire international de Paris, en 1878, qui devait principalement être consacré à la reconnaissance du droit de propriété littéraire. (Actes publiés par la Société des gens de lettres, à Paris, en 1879, p.104-111) 
œuvre, mais aussi le droit (mais non l'obligation) à une rémunération pour l'exploitation et la diffusion des œuvres pour les auteurs. Internet a libéré la circulation des œuvres culturelles, - soustraites de leur enveloppe matérielle - et cette révolution va de pair avec une liberté d'accès au savoir inégalée par son ampleur depuis Gutenberg. Mais, au sein de ce nouvel univers, pour des raisons principalement techniques, ni la reconnaissance de la paternité de l'œuvre (plagiat et contrefaçon facilitées) ni la rémunération ne sont plus totalement garanties (souffle de l'ère de la gratuité). C'est à cela que doit s'atteler la régulation, reconnaître et intégrer, par exemple, ces nouvelles formes de rémunération qui viennent en complément, et non de façons mutuellement exclusives, se substituer au droit d'auteur (ce qui est déjà le cas pour la majorité des auteurs de notre enquête). De même, reconnaître la nécessité de réformer le droit d'auteur - et non le principe de propriété intellectuelle reconnu comme universel64 - mais qui ne peut plus être reconduit à l'identique du fait de cette révolution disruptive. Quel est le sens de la publication sur Internet: s'agit-il de la mise en accès d'une chaîne de code sur un réseau ou de la construction d'une expression originale? II devient par exemple difficile de justifier aux yeux du public, ou de ceux qui parlent en son nom, l'allongement continu de la durée de protection des œuvres. Revenons à Victor Hugo: "Constatons la propriété littéraire, mais, en même temps, fondons le domaine public. Allons plus loin. Agrandissons-le. Que la loi donne à tous ${ }^{65}$ les éditeurs le droit de publier tous les livres après la mort des auteurs, à la seule condition de payer aux héritiers directs une redevance très faible "; sous quelles conditions est-il possible de revenir sur cette règle des soixante-dix ans après la mort, est-elle modulable 66 , comment traiter les œuvres orphelines, faut-il se

\footnotetext{
64 II existe un socle international commun en matière de droit d'auteur.

65 C'est nous qui soulignons.

66 Selon l'étude d'Ernst \& Young, The way to cultural diversity. The international survey on tax policies in the cultural sector?, qui vise notamment à établir une comparaison internationale des enjeux en matière de propriété intellectuelle dans les pays du G20, on constate que la convention de Berne protège les droits d'un auteur cinquante ans après son décès. Ce seuil minimal est en vigueur en Arabie Saoudite, au Canada, en Chine, en Corée du Sud et au Japon. II passe à soixante ans en Inde, à soixante-dix ans en Allemagne, en Australie,
} 
résoudre à leur oubli dès lors que les ayants-droits sont introuvables et les éditeurs non intéressés... Dès lors des adaptations s'imposent.

C'est un fait aujourd'hui, Internet a profondément modifié les pratiques de consommations des contenus culturels, la régulation se doit à présent de mettre en place des moyens qui assurent que celles-ci n'entravent pas la création culturelle. De nombreuses propositions s'attachent au financement partagé de la création: soutien aux plates-formes légales, extension de la licence légale, contribution créative ${ }^{67}$, mécénat global, culture du don (Proulx et Goldenberg, 2010)... II n'est pas ici le lieu de les discuter mais simplement de noter qu'elles reposent sur l'idée que le droit au partage et le droit à la culture doivent impliquer des contributions des lecteurs tout en se divisant sur les moyens et les formes de ces contributions ; elles sont confrontées à deux enjeux déterminants de la régulation: l'adaptation aux règles internationales (règles juridiques et règles du marché) et la fixation de justes contributions, à la fois des lecteurs, des auteurs et des intermédiaires. En d'autres termes, la clé de voûte du dispositif ne peut reposer sur la seule responsabilité de l'internaute. II reste aussi à adapter la régulation aux spécificités du livre, à un corps de créateurs très diversifiés, aux attentes parfois divergentes, accordant un poids très variable à la reconnaissance et à la rémunération. Et à replacer ces formes de contribution dans l'ensemble de la politique du livre, avec sa politique d'accompagnement, de commandes publiques, de soutiens des librairies, aux auteurs, aux écrits.

au Brésil, aux États-Unis, en France, en Italie, au Royaume-Uni, en Russie et en Turquie (durée qui prévaut désormais dans toute l'Europe désormais après l'application de la directive européenne du 29 octobre 1993), à cent ans au Mexique. Or, la notion de frontière géographie n'est plus nécessairement valide Iorsqu'il s'agit de circulation d'œuvre sur Internet.

67 Philippe Aigrain dans Internet et Création (2008) souligne l'importance de la multiplication des échanges d'œuvres numériques hors marché sur Internet. II s'interroge sur les nouvelles façons de rémunérer la création et propose de mettre en place une "contribution créative" qui consiste à autoriser les échanges hors marché d'œuvres numériques entre individus en mettant en place une contribution forfaitaire de tous les abonnés à Internet haut-débit pour rémunérer et financer la création. 


\section{Annexes}

\section{Annexe 1 : Bibliographie}

Adler, Moshe, "Stardom and Talent", American Economic Review, 1985, 75, p.208-212.

Aigrain, Philippe, Internet et Création. Comment reconnaître les échanges sur internet en finançant la création?, In Libro veritas, 2008.

Bacache-Beauvallet, Maya, Bourreau, Marc et Moreau, François, Portrait de musiciens à l'heure du numérique, Cepremap, Éditions rue d'Ulm, 2011.

Barluet, Sophie, Rapport : "Pour que vive la politique du livre ", juin 2007 dans le cadre de l'opération " Livre 2010 ", [en ligne].

Békir, Insaff, El Harbi, Sana et Grolleau, Gilles, "Le piratage peut-il être profitable à la firme piratée ", Revue économique, 2010, 4, 61, p. 793-805.

Benhamou, Françoise et Guillon, Olivia " Modèles économiques d'un marché naissant : le livre numérique ", Culture prospective, 2010, 2, p. 1-16 [en ligne]. Disponible sur www2.culture.gouv.fr/culture/deps/2008/pdf/cplivrenumerique-2010-2.pdf

Benhamou, Françoise, "L'industrie du livre ", in Cohen, Daniel et Verdier, Thierry, La mondialisation immatérielle, Rapport pour le Conseil d'Analyse Économique, La Documentation française, 2008, p. 73-115.

Benhamou, Françoise, L'Économie du star-système, Paris, Odile Jacob, 2002.

Benhamou, Françoise, L'Économie de la culture, Paris, La Découverte, 2003.

Bienvault, Hervé, Le coût d'un livre numérique : étude réalisée pour le MOTIF (Observatoire pour le livre et l'écrit en Ile-de-France), avril 2010 [en ligne]. Disponible sur http://www.lemotif.fr/

Boudon, Raymond, "L'intellectuel et ses publics: les singularités françaises ", in Reynaud Jean-Daniel et Grafmeyer Yves, Français qui êtes-vous ? Des essais et des chiffres, Paris, La Documentation française, 1981, p. 465-480. 
Carr, Nicholas, Is Google Making Us Stupid, Atlantic, juillet-août, 2008. Pour l'édition française, Internet rend-il stupide?, Paris, Robert Laffont, 2011.

Cardon, Dominique, La démocratie Internet. Promesses et limites, Paris, coédition Seuil - La République des idées, 2010.

Cardon, Dominique et Delaunay-Teterel, Hélène, " La production de soi comme technique relationnelle. Un essai de typologie des blogs par leurs publics ", Réseaux, 2006, 4, p. 15-71.

Chartier, Roger, L'Ordre des livres. Lecteurs, auteurs, bibliothèques en Europe entre le XIVe et XVIIle siècle, Aix-en-Provence, Alinéa, 1992.

Chartier, Roger, "Lecteurs et lectures à l'âge de la textualité électronique ", Centre Georges Pompidou, Colloque virtuel, octobre novembre 2001, www.text-e.org

Chartier, Roger, "Le livre : son passé, son avenir " : entretien avec Ivan Jablonka. La Vie des idées, 29 septembre 2008 [en ligne]. Disponible sur: http://www.laviedesidees.fr/Le-livre-sonpasse-son-avenir.html

Conner K, and Rumelt, R, “ Software piracy: an analysis of protection Strategies", Journal of Management Science, 1991, 37, 2, p. $125-139$.

Dacos, Marin et Mounier, Pierre, L'édition électronique ouverte: le carnet du Cléo, 2011 [en ligne]. Disponible sur: http://leo.hypotheses.org

Darnton, Robert , Apologie du livre : demain, aujourd'hui, hier, Paris, Gallimard, 2010.

Daval, Mathias, EbookZ 2 : étude sur l'offre numérique illégale des livres français sur Internet en 2010 - 2e année, MOTif, 2011.

Derrida, Jacques, La Carte postale. De Socrate à Freud et au-delà, Paris, Aubier-Flammarion, 1979.

Donnat, Olivier, Les Pratiques culturelles des Français à l'ère numérique : enquête 2008, Paris, La Découverte/Ministère de la Culture et de la Communication, 2009.

Dulong de Rosnay, Mélanie, La mise à disposition des œuvres et des informations sur les réseaux. Régulation juridique et régulation technique, Thèse de doctorat, Droit public, Université Panthéon Assas Paris II, octobre 2007.

Eisenstein, Elizabeth L, La Révolution de l'imprimé à l'aube de l'Europe moderne. Paris, La Découverte, 1991.

Epron, Benoît, L'édition universitaire française face au numérique : enjeux stratégiques et menaces économiques pour les manuels en sciences humaines et sociales, Thèse de doctorat en sciences de l'information et de la communication, Univer- 
sité Claude Bernard Lyon 1, 2005 [en ligne]. Disponible sur www.enssib.fr/bibliotheque-numerique/document-1374

Foucault, Michel, "Qu'est-ce qu'un auteur ? ", Dits et écrits, tome I, 1954-1988, Paris, Gallimard, 1994.

Goody, Jack, La Raison graphique, Paris, éditions de Minuit, 1979.

Gouyon, Marie, Diversité des carrières d'auteur de l'audiovisuel et du spectacle vivant. Les auteurs de la SACD, 1997-2008, avril 2011. http://www.culture.gouv.fr/nav/index-stat.html

Gouyon, Marie, Écrivains, photographes, compositeurs... Les artistes affiliés à l'Agessa en 2008. Étude socio-économique des auteurs affiliés à l'Agessa, avril 2011. http://www.culture.gouv.fr/nav/index-stat.html

Granovetter, Mark, Le marché autrement. Les réseaux dans l'économie, Paris, Desclée de Brouwer, 2000.

Guillaud, Hubert La Feuille : l'édition à l'heure de l'innovation [en ligne]. Disponible sur : http://lafeuille.blog.lemonde.fr

Han, I, and Croson, D, “Managing Software piracy: The role of Strategic Copy protection in Interfirm Competition ", 2000, Working paper, septembre.

Lahire, Bernard, La Condition littéraire: la double vie des écrivains, Paris, La Découverte, 2006.

Lebert, Marie, Le livre, de l'imprimé au numérique, Le Net des Études Françaises (NEF), août 2007.

Lessig, Lawrence, Code and other laws of cyberspace. New York, Basic Books, 1999.

MacDonald Glenn, "The Economics of Rising Stars ", American Economic Review, 78, 1988, p. 155-166.

Méadel, Cécile et Sonnac, Nathalie, “La fureur d'écrire. L'auteur au temps du numérique”, Esprit, à paraître, mai 2012.

Ministère de l'Éducation nationale Métamorphoses du livre et de la lecture à l'heure du numérique, séminaire du 22 au 24 novembre $2010 \quad$ [en ligne]. http://www.eduscol.education.fr/pid25134/seminairemetamorphoses-livre-lecture.html

Observatoire de l'économie du livre, Le secteur du livre: chiffres-clés 2009-2010, 2011-03-01.

Perona, Mathieu et Pouyet, Jérôme, Le Prix unique du livre à I'heure du numérique. Paris : Éd. Rue d'Ulm, 2010.

Pimlott, Herbert, "Eternal ephemera or the durability of "disposable literature': the power and persistence of print in an electronic world ", Media Culture \& Society, 33, 4, May 2011, p. 515530. 
Proulx, Serge et Goldenberg, Anne, "Internet et la culture de la gratuité”, Revue du Mauss, 2010, 35, 1, p. 503-517.

Robin, Christian, Les Livres dans l'univers numérique, Paris, La Documentation française, 2011.

Rosen, Sherwin, "The Economics of Superstars", American Economic Review 71, December 1981, p. 845-858.

Sapiro, Gisèle, "Mesures du littéraire ", Histoire \& Mesure, 23, 2, 2008, p. 35-68.

Sapiro, Gisèle et Gobille, Boris, "Propriétaires ou travailleurs intellectuels? " Les écrivains français en quête d'un statut, Le Mouvement Social, 1 214, 2006, p. 113-139

Shy, Oz, and Thisse, Jacques-François, "A strategic approach to software protection", Journal of Economics \& Management Strategy, 8, p. 163-190.

Slive, Jossua, and Berhardt, Dan, "Pirated for profit", Canadian Journal of Economics, 1988, 31, 4, p. 886-899.

Takeyama, I, "The Welfare Implications of unauthorized reproduction of Intellectual property in the presence of demand Network Externalities ", Journal of Industrial Economics, 1994, 62, p. 155-166.

Yvert, Benoit, "L'avenir du livre, entretien ", Le Débat, 2007 (3), $\mathrm{n}^{\circ} 145$, p.3-13.

Xerfi France, La Distribution de livres, Analyse de marché et perspectives 2010, Paris, Xerfi Editions, septembre 2010. 


\section{Annexe 2 : Liste des encadrés}

a) Le livre papier : définition juridique ....................................... 5

b) L'œuvre en collaboration ou œuvre plurale........................... 16

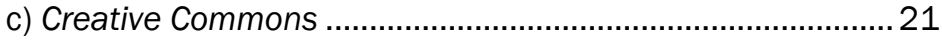

d) Le livre numérique : définition juridique ............................... 51

e) Brefs portraits d'auteurs dans le monde numérique ............ 84

f) Plagiat et Contrefaçon.............................................................. 99

g) Quelques éléments juridiques clés du Droit d'auteur.........108

h) Le droit de suite................................................................... 115

i) Étude de la SCAM sur la rémunération des auteurs par les éditeurs .................................................121

j) Baromètre 2010 des relations Auteurs / Éditeurs établi par la SCAM ................................................................ 127

k) La gestion collective des œuvres écrites ............................129 


\section{Annexe 3 : Liste des entretiens}

\begin{tabular}{|c|c|c|c|}
\hline $\begin{array}{c}\text { Pseudonyme utilisé dans } \\
\text { le rapport }\end{array}$ & Type de publications & Vit de sa plume & Localisation \\
\hline [Auteur-blogueur, Paris] & $\begin{array}{l}\text { Blogueur qui a publié des } \\
\text { romans en papier }\end{array}$ & Non & Paris \\
\hline [BD, Bretagne] & $\mathrm{BD}$ & Non & Brest \\
\hline$[\mathrm{BD}$, Paris $]$ & $\mathrm{BD}$ & Oui & Paris \\
\hline$[\mathrm{BD}, \mathrm{Sud}]$ & $\mathrm{BD}$ & Non & Sud \\
\hline $\begin{array}{c}\text { [Directeur d'ouvrage } \\
\text { collectif] }\end{array}$ & Essai (non fiction) & Non & Paris \\
\hline [Homme de lettres, Paris] & $\begin{array}{c}\text { Romans, essais, BD, films } \\
\text { documentaires }\end{array}$ & $\begin{array}{l}\text { Oui + activité } \\
\text { d'éditeur }\end{array}$ & Paris/ Bruxelles \\
\hline [Jeune auteur, Paris] & Roman & Non & Paris \\
\hline [Littérateur, Paris] & $\begin{array}{c}\text { Romans/ pièces de } \\
\text { théâtre }\end{array}$ & Non & Paris \\
\hline $\begin{array}{l}\text { [Littérature enfantine, } \\
\text { Ajaccio] }\end{array}$ & $\begin{array}{l}\text { Littérature pour enfants } \\
\text { (principalement) }\end{array}$ & Non & Ajaccio \\
\hline $\begin{array}{c}\text { [Littérature enfantine, } \\
\text { Paris] }\end{array}$ & Littérature pour enfants & Oui & Paris \\
\hline $\begin{array}{l}\text { [Médecin, Roman poli- } \\
\text { cier, Paris] }\end{array}$ & Roman policier & Non & Paris \\
\hline [Nutrition, Paris] & $\begin{array}{c}\text { Pratique (ouvrages de } \\
\text { nutrition) }\end{array}$ & Non & Paris \\
\hline [Philosophe, Paris] & $\begin{array}{c}\text { Sciences humaines } \\
\text { (ouvrages de philosophie) }\end{array}$ & Non & Paris \\
\hline $\begin{array}{c}\text { [Policier, Roman policier, } \\
\text { Paris] }\end{array}$ & Roman policier & Non & Paris \\
\hline [Premier roman, Paris] & Premier roman & Non & Paris \\
\hline $\begin{array}{l}\text { [Roman historique, Sud- } \\
\text { Ouest] }\end{array}$ & Romans historiques & Non & Toulouse \\
\hline [Romancier, Paris] & Roman & Non & Paris \\
\hline [Romancier, Picardie] & Premier roman & Non & Picardie \\
\hline
\end{tabular}




\begin{tabular}{|c|c|c|c|}
\hline $\begin{array}{c}\text { Pseudonyme utilisé dans } \\
\text { le rapport }\end{array}$ & Type de publications & Vit de sa plume & Localisation \\
\hline $\begin{array}{c}\text { [Romancière, Communi- } \\
\text { cation, Paris] }\end{array}$ & Roman & Oui & Paris \\
\hline $\begin{array}{c}\text { [Science-fiction, Angle- } \\
\text { terre] }\end{array}$ & Science-fiction & Oui & Angleterre \\
\hline $\begin{array}{c}\text { [Sciences humaines, } \\
\text { États-Unis] }\end{array}$ & $\begin{array}{c}\text { Sciences humaines } \\
\text { (Ouvrages sur les médias } \\
\text { et technologies de } \\
\text { l'information) }\end{array}$ & Paris \\
\hline [Vulgarisation, Paris] & $\begin{array}{c}\text { Pratique (ouvrages de } \\
\text { culture générale) }\end{array}$ & $\begin{array}{c}\text { Oui + activité } \\
\text { d'éditeur }\end{array}$ & \\
\hline
\end{tabular}




\section{Annexe 4 : La trame de l'entretien}

Comment les auteurs s'inscrivent dans l'espace du livre reconfiguré par le numérique? II ne s'agit pas ici d'un questionnaire fermé, mais d'un aide-mémoire des thèmes et types de questionnement, commun aux auteurs de ce livre qui ont tous participé à la campagne d'entretiens.

1. Sa carrière d'auteur: quelles sont vos activités en tant qu'auteur? combien de livres (écrits, publiés...) ? depuis quand écrivez-vous ? Combien de temps consacrez-vous à écrire ? Est-ce votre activité principale? Depuis quand êtes-vous publié ? Vos livres ont-ils connu plusieurs éditions, des éditions en poche? Vos activités d'écrivain vous ont-elles conduites sur le web ? si oui comment?

2. Son rapport à l'éditeur: toujours le même éditeur? si vous en avez changé pourquoi ? comment avezvous pris contact avec lui ? Aviez-vous déposé votre œuvre avant? L'avez-vous fait après ? Quelle est le rôle de l'éditeur par rapport à votre manuscrit (relecture, mise en page, contenu, choix du titre...). L'échange avec votre éditeur vous paraît-il équilibré ? Vous êtes-vous déjà autoproduit (à compte d'auteur, mise en ligne sur un site...) ? Avez-vous des textes en ligne?

3. La vie du manuscrit : Comment écrivez-vous ? Tapezvous votre texte vous-même? Fournissez-vous à votre éditeur un fichier numérique ? Intervenez-vous dans la maquette, la mise en page et la numérisation de votre manuscrit? Quelle est le rôle de l'éditeur par rapport à votre manuscrit (relecture, mise en page, contenu, choix du titre...) ? Que pensez-vous des modifications proposées par votre éditeur? 
4. La diffusion du livre : participez-vous à des salons ? faites-vous des lectures publiques? Rencontrezvous les représentants, les libraires, les journalistes, l'attaché de presse ? Votre éditeur a-t-il un service presse ? Qu'en pensez-vous ? Avez-vous un blog, un site, une page Facebook? (et si oui qu'en faitesvous ?) Avez-vous des occasions de rencontrer des lecteurs? Qui sont vos lecteurs? Votre livre pourrait-il être mieux diffusé ? Quels sont les obstacles à la diffusion? Le numérique (si les œuvres sont sous forme numérique) permet-il d'autres façons de vendre l'œuvre? Comment êtes-vous présent (si vous l'êtes) sur le web?

5. La notion de droit d'auteur: si je vous dis droit d'auteur, quelles sont toutes les choses auxquelles vous pensez? Quels sont pour vous les avantages et les inconvénients du droit d'auteur? finalement, quel est le rôle du droit d'auteur? le droit d'auteur, c'est comme quoi... ce n'est pas du tout comme quoi...

6. Le contrat d'auteur? Connaissez-vous les conditions de votre contrat (pourcentage, nombre d'exemplaires donnés à l'auteur, tirage...) Estimez-vous cette rémunération équitable? Avez-vous sollicité des modifications ? avez-vous plusieurs contrats ? quelles différences? Connaissez-vous la durée de votre contrat (souvent la durée des droits) ? Y a-t-il des clauses particulières (pour vos ouvrages suivants, pour les nouvelles éditions, pour les nouveaux tirages) ? Combien d'exemplaires auteurs avez-vous? Savez-vous s'il y a des clauses pour les droits annexes (étrangers et numériques); si oui ont-ils été négociés à part? Êtes-vous rémunéré au titre du droit de prêt (SOFIA) et du droit de reprographie?

7. La vie en numérique (approche prospective): Qu'évoque pour vous le numérique? Change-t-il votre façon d'écrire? Vos relations avec votre éditeur, avec votre public ? Avec d'autres acteurs du livre? (Pour ceux dont les textes ne sont pas en 
ligne) : comment réagiriez-vous à la mise en ligne de vos ouvrages sous forme de livre électronique? les avantages et inconvénients du livre numérique pour vous, pour votre éditeur, pour le lecteur? Êtes-vous intéressé par un livre enrichi (par des documents en ligne, des fichiers, sonores, vidéo...) ? Utilisez-vous les livres en ligne? Êtes-vous sur Google books? La numérisation permet de découper des œuvres, de les modifier, de les enrichir: quels usages seriezvous prêt à accepter? Quels types d'usages êtesvous prêts à accepter (usages gratuits, moins chers, usages académiques ou pédagogiques) ? Quid des exceptions au droit d'auteur? Qu'est-ce qu'un livre pour vous aujourd'hui et demain? Le numérique change-t-il ce qu'est être un auteur ? Cela change-t-il ce qu'est une œuvre ? Change-t-il le droit d'auteur? En quoi et pour quelles raisons? Quelle régulation de la diffusion électronique du livre souhaitez-vous? Que pensez-vous de la loi Hadopi et du principe de réponse graduée?

8. Les revenus : vivez-vous de vos œuvres ? Avez-vous une autre source de revenus? Travaillez-vous pour des éditeurs (comment) ? Vos revenus d'auteur proviennent-ils uniquement des droits d'auteurs? (commande publique ou autres aides institutionnelles, invitation, prix, commandes privées, création radiophonique, atelier d'écriture...). Êtes-vous membre des sociétés d'auteur (lesquelles) ? 


\section{Sommaire}

Intraduction .............................................................................

L'enquête............................................................................. 10

\section{Chapitre $1:$}

\section{Écrire en régime numérique .............................................19}

1.1 Écrire et fabriquer un livre ............................................. 15

La démarche d'écriture ................................................... 15

Le manuscrit.................................................................32

La fabrication du livre.................................................... 36

Conclusion intermédiaire : le dialogue des supports .......42

1.2 L'objet livre reconfiguré...................................................... 44

Le livre " en continu " ........................................................ 44

Le livre " enrichi "............................................................... 46

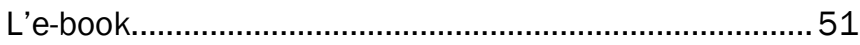

Conclusion intermédiaire : Vers un livre numérique ........56

1.3 Internet comme lieu d'apprentissage .............................57

L'auteur en réseau ........................................................57

Un outil de recherche et un " baromètre " des temps.....59

Internet " facilitateur " ....................................................... 60

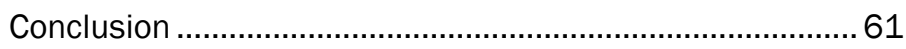

\section{Chapitre 르 :}

\section{Internet dans la diffusion,}

la diffusion hars Internet..................................................63

2.1 La diffusion hors Internet : les canaux traditionnels..... 64 Les rencontres avec le public ........................................... 66

2.2 La diffusion en ligne : outils et modalités ...................... 71

De l'obligation d'y être et de son coût.................................. 71

Des divers dispositifs mobilisés ....................................... 75 
Internet comme moyen d'échanger avec les lecteurs ....78 Conclusion intermédiaire : portraits de l'auteur comme internaute

2.3 Un nouveau rôle pour l'auteur ? ....................................... 87

L'émergence d'un nouveau profil d'auteur ?.................... 87

Internet, un vieux média tout neuf ? ................................ 89

Les œuvres collaboratives et évolutives ..........................99

2.4 Le piratage, entre notoriété et rémunération .................94

Genres, notoriété et droit d'auteur...................................94 94

Ambivalence vis-à-vis du piratage .....................................97

Conclusion intermédiaire : le partage de la valeur ............103

\section{Chapilte $\exists$}

\section{Les relations Auteurs - Éditeurs à I'ère du numérique}

3.1 La recherche par l'auteur d'un " partenaire " ..............109

Comment établir la confiance........................................113

Un soutien plus ou moins investi dans le travail de création ..........................................................................116

L'éditeur comme médiateur ............................................118

3.2 Des relations contractuelles .......................................120

Les droits directs ............................................................121

Un rapport de force asymétrique..................................125

Les droits secondaires .................................................129

Le prix du livre comme marqueur..................................134

Le statut de l'auteur en question ..................................136

3.3 L'éditeur et le numérique...........................................138

Une désintermédiation largement récusée ...................141

Vers de nouveaux intermédiaires ?................................144

Vers un déplacement du risque lié à la publication ?...146

Conclusion : le numérique déplace l'asymétrie, mais la rééquilibre-t-il ? 
Le numérique, vers un nouvel espace de création pour

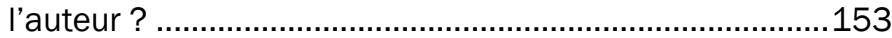

L'auteur en sa propriété .................................................156

Annex

Annexe 1 : Bibliographie ...................................................163

Annexe 2 : Liste des encadrés .........................................167

Annexe 3 : Liste des entretiens ........................................169

Annexe 4 : La trame de l'entretien....................................171 


\section{L'auteur \\ au temps du numérique}

sous la direction de : Cécile Méadel et Nathalie Sonnac

Avec : Audrey Llamas, Francesca Musiani, Mathieu Perona, Mathilde Persuy,

Vincent Petitet, Marianne Serfaty, Guillaume Sire, Virginie Sonet

Que signifie aujourd'hui l'acte d'écrire et de publier un livre ? Quelle influence ont la numérisation des contenus, le développement d'Internet et les nouvelles pratiques de lecture sur le travail des auteurs ? Pour répondre à ces questions, ont été interrogés une variété d'auteurs dans toute leur diversité de producteurs d'ouvrages : des auteurs de bande dessinée aux concepteurs de livres pratiques, en passant par ceux qui écrivent des fictions ou des romans policiers. II ne s'agit pas d'opposer deux univers, l'un attaché à la seule écriture papier et l'autre investi dans une dynamique dématérialisée du tout numérique, mais de comprendre comment les deux se complètent et se composent, comment le numérique ouvre de nouvelles façons d'écrire et offre de nouvelles possibilités d'édition et de publication, comment il permet d'intervenir sur le colloque singulier entre l'auteur et le lecteur, sur les échanges entre les producteurs et le public, sur la relation entre l'auteur et l'éditeur. Mais aussi comment le livre-papier garde pour les auteurs une place décisive et peut-être indétrônable.

Cette étude a été menée dans le cadre des recherches de la Haute Autorité pour la diffusion des œuvres et la protection des droits sur Internet qui a pour mission d'étudier l'univers des industries culturelles et médiatiques et de produire en toute indépendance débats et réflexions sur les questions liées à la diffusion numérique et à la propriété intellectuelle. Elle a été dirigée par Cécile Méadel, professeure à Mines ParisTech, et Nathalie Sonnac, professeure à l'Université Panthéon Assas, respectivement expertes des Labs Hadopi Usages en Ligne et Économie Numérique de la Création.

ISBN : 9782813000880
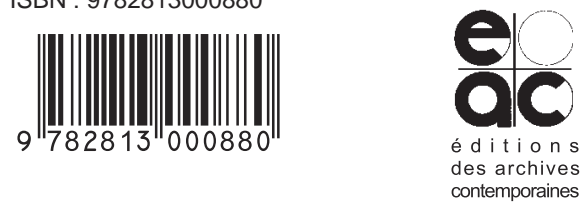

LabsHadopi 